\title{
Carleman estimates for time-discrete parabolic equations and applications to controllability
}

\author{
Franck Boyer \\ Víctor Hernández-Santamaría
}

May 6, 2019

\begin{abstract}
In this paper, we prove a Carleman estimate for a time-discrete parabolic equation in which the large parameter is connected to the time step of the discretization scheme. This estimate is then used to obtain a relaxed observability estimate that yields some controllability results for linear and semi-linear time-discrete parabolic equations. We also discuss the application of this Carleman estimate to the controllability of coupled systems.
\end{abstract}

Keywords: Carleman estimates, time-discrete heat equation, observability, null controllability.

\section{Introduction}

\section{$1.1 \quad$ Null-controllability of the heat equation}

Let $\Omega \subset \mathbb{R}^{d}, d \geq 1$ be a bounded open set with boundary $\partial \Omega$ regular enough. For given time $T>0$, we denote $Q=\Omega \times(0, T)$ and $\Sigma=\partial \Omega \times(0, T)$. Let $\omega$ be a nonempty subset of $\Omega$. We consider the linear heat equation

$$
\begin{cases}\partial_{t} y-\Delta y=\mathbf{1}_{\omega} v & \text { in } Q, \\ y=0 & \text { on } \Sigma, \\ y(x, 0)=y_{0}(x) & \text { in } \Omega .\end{cases}
$$

In (1.1), $y=y(x, t)$ is the state, $v=v(x, t)$ is the control function acting on the system on the control domain $\omega$, and $y_{0}$ is a given initial data. As usual, $\mathbf{1}_{\omega}$ denotes the characteristic function of $\omega$.

It is well-known that for any $y_{0} \in L^{2}(\Omega)$ and $v \in L^{2}(\omega \times(0, T))$, the corresponding solution to (1.1) is globally defined in $[0, T]$. More precisely, one has

$$
y \in C\left([0, T] ; L^{2}(\Omega)\right) \cap L^{2}\left(0, T ; H_{0}^{1}(\Omega)\right) .
$$

One of the key questions in control theory is to determine whether a system enjoys the so-called null controllability property. System (1.1) is said to be null-controllable at time $T$ if, for any $y_{0} \in L^{2}(\Omega)$, there exists a control $v \in L^{2}(\omega \times(0, T))$ such that the corresponding solution satisfies

$$
y(\cdot, T)=0 \quad \text { in } \Omega .
$$

Observe that the regularity (1.2) justifies the definition we have introduced.

It is by now well-known that (1.1) is null-controllable for any $T>0$ and for any nonempty open set $\omega \subset \Omega$. In fact, this problem was addressed independently in the 90's by Lebeau \& Robbiano [15] and by Fursikov \& Imanuvilov [12]. By a duality argument, the null-controllability of (1.1) is equivalent to the observability of the associated adjoint states. More precisely, for each $q_{T} \in L^{2}(\Omega)$, consider the adjoint system

$$
\begin{cases}-\partial_{t} q-\Delta q=0 & \text { in } Q \\ q=0 & \text { on } \Sigma \\ q(x, T)=q_{T}(x) & \text { in } \Omega .\end{cases}
$$


Then, (1.1) is null-controllable if and only if there exists $C_{o b s}>0$ such that the following observability inequality holds

$$
|q(0)|_{L^{2}(\Omega)} \leq C_{o b s}\left(\iint_{\omega \times(0, T)}|q|^{2} d x d t\right)^{\frac{1}{2}}, \quad \forall q_{T} \in L^{2}(\Omega)
$$

\section{$1.2 \quad$ Time-discrete setting}

In this paper, we shall use the notation $\llbracket a, b \rrbracket=[a, b] \cap \mathbb{N}$, for any real numbers $a<b$.

We are interested in studying controllability and observability properties for the time-discrete counterparts to (1.1) and (1.3), but also for more general parabolic systems (see Sections 4 and 5 ). To be more precise, for any given $M \in \mathbb{N}^{*}$, we set $\triangle t=T / M$ and introduce the following discretization for the time variable

$$
0=t_{0}<t_{1}<\ldots<t_{M}=T,
$$

with $t_{n}=n \triangle t$ and $n \in \llbracket 0, M \rrbracket$. We also introduce $t_{n+\frac{1}{2}}=\left(t_{n+1}+t_{n}\right) / 2$, for $n \in \llbracket 0, M-1 \rrbracket$, see Figure 1 .

For any time discrete control sequence $v=\left\{v^{n+\frac{1}{2}}\right\}_{n \in \llbracket 0, M-1 \rrbracket} \subset L^{2}(\Omega)$, consider the sequence $y=$ $\left\{y^{n}\right\}_{n \in \llbracket 0, M \rrbracket} \subset L^{2}(\Omega)$ verifying the recursive formula

$$
\begin{cases}\frac{y^{n+1}-y^{n}}{\triangle t}-\Delta y^{n+1}=\mathbf{1}_{\omega} v^{n+\frac{1}{2}}, & n \in \llbracket 0, M-1 \rrbracket, \\ y_{\mid \partial \Omega}^{n+1}=0, & n \in \llbracket 0, M-1 \rrbracket \\ y^{0}=y_{0} & \end{cases}
$$

where $y^{n}$ (resp. $\left.v^{n+\frac{1}{2}}\right)$ denotes an approximation of $y$ (resp. $v$ ) at time $t_{n}$ (resp. $t_{n+\frac{1}{2}}$ ). Observe that (1.6) is precisely an implicit Euler discretization of the heat equation. Evidently, there exist many other methods to discretize (1.1), but we have chosen this method for the sake of simplicity.

As in the continuous case, we can introduce the notion of controllability for the discrete scheme, this is, system (1.6) is said to be null controllable if for any $y_{0} \in L^{2}(\Omega)$ there exists a sequence $\left\{v^{n+\frac{1}{2}}\right\}_{n \in \llbracket 0, M-1 \rrbracket}$ such that the corresponding solution satisfies

$$
y^{M}=0
$$

Notice that for any fixed $\triangle t$ and for each $n \in \llbracket 0, M-1 \rrbracket,(1.6)$ can be regarded as a system of controlled elliptic equations.

There are only a handful of works in the literature addressing the controllability of time-discrete systems such as (1.6). This may come from the fact that, as pointed out in [22, Theorem 1.1], system (1.6) is not even approximately controllable for any given $\Delta t>0$, except for the trivial case when $\omega=\Omega$. In view of this negative result, it is natural to ask whether the controllability requirement (1.7) for system (1.6) can be relaxed. In this direction, in [22], the controllability for (1.6) is achieved by considering the projections of solutions over a suitable class of low frequency Fourier components. Using a time-discretized Lebeau-Robbiano strategy (see e.g. $[15,16]$ ), the author proves a uniform controllability result (with respect to $\triangle t$ ) for the low frequency part of the solution.

In [7], the authors prove in a quite general framework that any controllable parabolic equation (even if discretized in the space variable) is null controllable after time discretization by an appropriate filtering of the high-frequencies. In [5], the authors study in a general setting the null-controllability of fullydiscrete approximations for parabolic equations and its convergence rate towards the semi-discrete (in space) case. There, the authors prove some relaxed observability estimates (uniform with respect to the discretization parameters) allowing to recover classical results for the continuous case. However, both of these works rely on spectral analysis tools and therefore the results are limited to autonomous linear control systems. We finally mention the works [8, 21, 23] which encompass the controllability of time discretization schemes for wave-like, Schrödinger and KdV equations.

Here, inspired in the strategy outlined in [6] (see also [4]) for the space discretization of parabolic equations, we derivate a Carleman estimate for time-discrete approximations of the parabolic operator $-\partial_{t}-\Delta$ and from there we deduce a relaxed observability inequality for a suitable adjoint system. We shall refer to it as a relaxed inequality due to the presence of an extra term on the right-hand side (as 


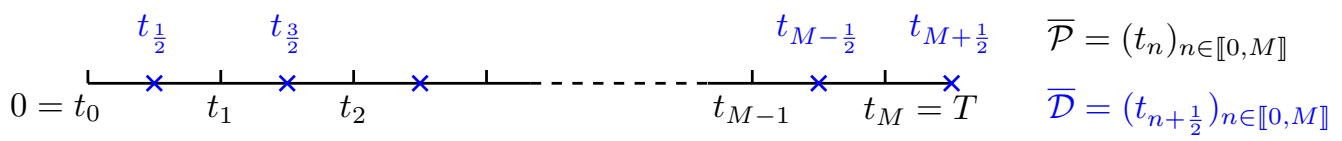

Figure 1: Discretization of the time variable and its notation.

compared to (1.4)). This inequality, in turn, allows to obtain by duality a controllability result where a small target is reached and whose size goes to zero exponentially as $\Delta t \rightarrow 0$, more precisely, we achieve

$$
\left|y^{M}\right|_{L^{2}(\Omega)} \leq C_{o b s} \sqrt{\phi(\triangle t)}\left|y_{0}\right|_{L^{2}(\Omega)},
$$

where $C_{o b s}$ is a positive constant uniform with respect to $\triangle t$ and $\triangle t \mapsto \phi(\triangle t)$ is a suitable function of the discretization parameter $\triangle t$. For this reason, in the spirit of [6], we shall speak of a $\phi(\triangle t)$-null controllability result.

The main goal and novelty of our approach are twofold. By deriving directly a Carleman estimate for the time-discrete operator, we can deduce controllability results for more general systems (e.g., equations with time-dependent coefficients, right-hand side terms, etc) since we are no longer restricted to spectral techniques (as in $[7,5,22]$ ). Moreover, our methodology can be readily adapted to derive the analogous counterpart of well-known controllability results in the continuous case, commonly relying on Carleman inequalities, such as the cases of semilinear systems, coupled equations, or non-standard problems as the insensitizing control.

\subsection{Notations and functional framework}

Before introducing our main results, we establish the framework of the discrete setting we shall work with. The notation introduced here allow us to use a formalism as close as possible to the continuous case and, in this way, most of the computations will be carried out in a very intuitive manner.

From the discretization points (1.5), we will denote by $\mathcal{P}:=\left\{t_{n}: n \in \llbracket 1, M \rrbracket\right\}$ the (primal) set of points excluding the first one and we write $\overline{\mathcal{P}}:=\mathcal{P} \cup\left\{t_{0}\right\}$. To handle in an efficient way computations related to the approximation of the time derivatives, we will naturally work on another (dual) set of points lying at the middle points of $\mathcal{P}$. More precisely, we define $\mathcal{D}:=\left\{t_{n+\frac{1}{2}}: n \in \llbracket 0, M-1 \rrbracket\right\}$. It will be convenient to consider also an extra point $t_{M+\frac{1}{2}}$ which lies outside the interval $[0, T]$ (see Figure 1) and to write $\overline{\mathcal{D}}:=\mathcal{D} \cup\left\{T_{M+\frac{1}{2}}\right\}$. Observe that both $\mathcal{P}$ and $\mathcal{D}$ have a total number of $M$ elements.

We denote by $\mathbb{R}^{\mathcal{P}}$ and $\mathbb{R}^{\mathcal{D}}$ the sets of real-valued discrete functions defined on $\mathcal{P}$ and $\mathcal{D}$. If $u^{\mathcal{P}} \in \mathbb{R}^{\mathcal{P}}$ $\left(\right.$ resp. $u^{\mathcal{D}} \in \mathbb{R}^{\mathcal{D}}$ ), we denote by $u^{n}$ (resp. $u^{n+\frac{1}{2}}$ ) its value corresponding to $t_{n}$ (resp. $t_{n+\frac{1}{2}}$ ). For $u^{\mathcal{P}} \in \mathbb{R}^{\mathcal{P}}$ we define the time-discrete integral

$$
\int_{0}^{T} u^{\mathcal{P}}:=\sum_{n=1}^{M} \triangle t u^{n}
$$

and, analogously, for $u^{\mathcal{D}} \in \mathbb{R}^{\mathcal{D}}$ we define

$$
\int_{0}^{T} u^{\mathcal{D}}:=\sum_{n=0}^{M-1} \Delta t u^{n+\frac{1}{2}}
$$

Remark 1.1. To ease the notation and thanks to the introduction of two different integral symbols, in what follows we shall write $u$ indistinctly to refer to functions $u^{\mathcal{P}}$ or $u^{\mathcal{D}}$.

Let $\{X,|\cdot| X\}$ be a real Banach space. We denote by $X^{\mathcal{P}}$ and $X^{\mathcal{D}}$ the sets of vector-valued functions defined on $\mathcal{P}$ and $\mathcal{D}$, respectively. Using the definitions (1.8) and (1.9) for the discrete integrals, we denote by $L_{\mathcal{P}}^{p}(0, T ; X)$ (resp. $\left.L_{\mathcal{D}}^{p}(0, T ; X)\right), 1 \leq p<\infty$, the space $X^{\mathcal{P}}\left(\operatorname{resp} . X^{\mathcal{D}}\right)$ endowed with the norm

$$
\|u\|_{L_{\mathcal{P}}^{p}(0, T ; X)}:=\left(\int_{0}^{T}|u|_{X}^{p}\right)^{1 / p} \quad\left(\operatorname{resp} . \quad\|u\|_{L_{\mathcal{D}}^{p}(0, T ; X)}:=\left(f_{0}^{T}|u|_{X}^{p}\right)^{1 / p}\right) .
$$


We also define the space $L_{\mathcal{P}}^{\infty}(0, T ; X)$ (resp. $\left.L_{\mathcal{D}}^{\infty}(0, T ; X)\right)$ by means of the norm

$$
\|u\|_{L_{\mathcal{P}}^{\infty}(0, T ; X)}:=\sup _{t_{n} \in \mathcal{P}}\left|u^{n}\right|_{X} \quad\left(\operatorname{resp.} \quad\|u\|_{L_{\mathcal{D}}^{\infty}(0, T ; X)}:=\sup _{t_{n+\frac{1}{2}} \in \mathcal{D}}\left|u^{n+\frac{1}{2}}\right|_{X}\right) .
$$

In the case where $p=2$ and $X$ is replaced by a Hilbert space $\left\{H,(\cdot, \cdot)_{H}\right\}, H^{\mathcal{P}}$ (resp. $H^{\mathcal{D}}$ ) becomes a Hilbert space for the norm induced by the inner product

$$
\int_{0}^{T}(u, v)_{H}:=\sum_{n=1}^{M} \triangle t\left(u^{n}, v^{n}\right)_{H} \quad\left(\operatorname{resp} . \quad f_{0}^{T}(u, v)_{H}:=\sum_{n=0}^{M-1} \Delta t\left(u^{n+\frac{1}{2}}, v^{n+\frac{1}{2}}\right)_{H}\right) .
$$

Particularly, if $H=L^{2}(\Omega)$ we shall use the notation

$$
\iint_{Q} u v:=\int_{0}^{T}(u, v)_{L^{2}(\Omega)} \quad\left(\operatorname{resp} . \quad f \int_{Q} u v:=f_{0}^{T}(u, v)_{L^{2}(\Omega)}\right) .
$$

Remark 1.2. For short and in accordance with the notation used in the continuous case, we will denote the spaces $L_{\mathcal{P}}^{2}\left(0, T ; L^{2}(\Omega)\right)$ as $L_{\mathcal{P}}^{2}(Q)$ and we use $L_{\mathcal{P}}^{\infty}(Q)$ to indicate the space $L_{\mathcal{P}}^{\infty}\left(0, T ; L^{\infty}(\Omega)\right)$. The same notation holds for functions defined on the dual grid $\mathcal{D}$.

To manipulate time-discrete functions, we define translation operators for indices $\mathrm{t}^{+}: X^{\mathcal{P}} \rightarrow X^{\mathcal{D}}$ and $\mathrm{t}^{-}: X^{\overline{\mathcal{P}}} \rightarrow X^{\mathcal{D}}$ as follows:

$$
\left(\mathrm{t}^{+} u\right)^{n+\frac{1}{2}}:=u^{n+1}, \quad\left(\mathrm{t}^{-} u\right)^{n+\frac{1}{2}}:=u^{n}, \quad n \in \llbracket 0, M-1 \rrbracket .
$$

With this, we can define a difference operator $D_{t}$ as the map from $X^{\overline{\mathcal{P}}}$ into $X^{\mathcal{D}}$ given by

$$
\left(D_{t} u\right)^{n+\frac{1}{2}}:=\frac{u^{n+1}-u^{n}}{\triangle t}=\left(\frac{1}{\triangle t}\left(\mathrm{t}^{+}-\mathrm{t}^{-}\right) u\right)^{n+\frac{1}{2}}, \quad n \in \llbracket 0, M-1 \rrbracket .
$$

In the same manner, we can define the translation operators $\overline{\mathrm{t}}^{+}: X^{\overline{\mathcal{D}}} \rightarrow X^{\mathcal{P}}$ and $\overline{\mathrm{t}}^{-}: X^{\mathcal{D}} \rightarrow X^{\mathcal{P}}$ as follows:

$$
\left(\overline{\mathrm{t}}^{+} u\right)^{n}:=u^{n+\frac{1}{2}}, \quad\left(\overline{\mathrm{t}}^{-} u\right)^{n}=u^{n-\frac{1}{2}}, \quad n \in \llbracket 1, M \rrbracket,
$$

as well as a difference operator $\bar{D}_{t}$ (mapping $X^{\overline{\mathcal{D}}}$ into $X^{\mathcal{P}}$ ) given by

$$
\left(\bar{D}_{t} u\right)^{n}:=\frac{u^{n+\frac{1}{2}}-u^{n-\frac{1}{2}}}{\triangle t}=\left(\frac{1}{\triangle t}\left(\overline{\mathrm{t}}^{+}-\overline{\mathrm{t}}^{-}\right) u\right)^{n}, \quad n \in \llbracket 1, M \rrbracket .
$$

These definitions, together with the integral symbols (1.8) and (1.9), allow us to obtain a series of results for handling in quite natural fashion the application of the derivatives $D_{t}$ and $\bar{D}_{t}$ to functions either continuously defined or discrete. For convenience, we have summarized in Appendix B the main tools and estimates used along this document. As an example, for functions $u \in\left[L^{2}(\Omega)\right]^{\overline{\mathcal{P}}}$ and $v \in\left[L^{2}(\Omega)\right]^{\overline{\mathcal{D}}}$, we have the following useful formula

$$
f \int_{Q}\left(D_{t} u\right) v=-\left(u^{0}, v^{\frac{1}{2}}\right)_{L^{2}(\Omega)}+\left(u^{M}, v^{M+\frac{1}{2}}\right)_{L^{2}(\Omega)}-\iint_{Q}\left(\bar{D}_{t} v\right) u
$$

which resembles classical integration by parts. Expressions like (1.11) allow us to present and perform computations intuitively, facilitating also the presentation and reading of this paper.

\subsection{Statement of the main results}

\subsubsection{Carleman estimate}

Let us introduce several weight functions that will be useful in the remainder of this paper. We introduce a special function whose existence is guaranteed by the following result [12, Lemma 1.1]. 
Lemma 1.3. Let $\mathcal{B}_{0} \subset \subset \Omega$ be a nonempty open subset. Then, there exists $\psi \in C^{2}(\bar{\Omega})$ such that

$$
\begin{cases}\psi(x)>0 & \text { all } x \in \Omega, \\ |\nabla \psi|>0 & \text { for all } x \in \overline{\Omega \backslash \mathcal{B}_{0}}\end{cases}
$$

Let $K>\|\psi\|_{C(\bar{\Omega})}$ and set

$$
\varphi(x)=e^{\lambda \psi(x)}-e^{\lambda K}<0, \quad \phi(x)=e^{\lambda \psi(x)},
$$

and

$$
\theta(t)=\frac{1}{(t+\delta T)(T+\delta T-t)} .
$$

for some $0<\delta<1 / 2$. The parameter $\delta$ is introduced to avoid singularities at time $t=0$ and $t=T$ (see Remark 1.5 below for further comments).

We state our first result, a uniform Carleman estimate for the time-discrete backward parabolic operator formally defined on the dual grid as follows

$$
\left(L_{\mathcal{D}} q\right)^{n}:=-\left(\bar{D}_{t} q\right)^{n}-\Delta\left(\overline{\mathrm{t}}^{-} q\right)^{n}, \quad n \in \llbracket 1, M \rrbracket,
$$

for any $q \in\left(H^{2}(\Omega)\right)^{\overline{\mathcal{D}}}$. The result is the following.

Theorem 1.4. Let $\mathcal{B}_{0}$ be a nonempty open set of $\Omega$ and a function $\psi$ provided by Lemma 1.3 and define $\varphi$ according to (1.12). Let $\mathcal{B}$ another open subset of $\Omega$ such that $\mathcal{B}_{0} \subset \subset \mathcal{B}$. For the parameter $\lambda \geq 1$ sufficiently large, there exist $C, \tau_{0} \geq 1, \varepsilon_{0}>0$, depending on $\mathcal{B}, \mathcal{B}_{0}, T$ and $\lambda$ such that

$$
\begin{aligned}
& \tau^{-1} \iint_{Q} \overline{\mathrm{t}}^{-}\left(e^{2 \tau \theta \varphi} \theta^{-1}\right)\left(\left|\bar{D}_{t} q\right|^{2}+\left|\Delta\left(\overline{\mathrm{t}}^{-} q\right)\right|^{2}\right)+\tau \iint_{Q} \overline{\mathrm{t}}^{-}\left(e^{2 \tau \theta \varphi} \theta\right)\left|\nabla\left(\overline{\mathrm{t}}^{-} q\right)\right|^{2}+\tau^{3} \iint_{Q} \overline{\mathrm{t}}^{-}\left(e^{2 \tau \theta \varphi} \theta^{3}\right)\left(\overline{\mathrm{t}}^{-} q\right)^{2} \\
& \leq C\left(\iint_{Q}\left(\overline{\mathrm{t}}^{-} e^{2 \tau \theta \varphi}\right)\left|L_{\mathcal{D}} q\right|^{2}+\tau^{3} \iint_{\mathcal{B} \times(0, T)} \overline{\mathrm{t}}^{-}\left(e^{2 \tau \theta \varphi} \theta^{3}\right)\left(\overline{\mathrm{t}}^{-} q\right)^{2}\right) \\
&+C(\triangle t)^{-1}\left(\int_{\Omega}\left|\left(e^{\tau \theta \varphi} q\right)^{\frac{1}{2}}\right|^{2}+\int_{\Omega}\left|\left(e^{\tau \theta \varphi} q\right)^{M+\frac{1}{2}}\right|^{2}+\int_{\Omega}\left|\left(e^{\tau \theta \varphi} \nabla q\right)^{M+\frac{1}{2}}\right|^{2}\right),
\end{aligned}
$$

for all $\tau \geq \tau_{0}\left(T+T^{2}\right)$, and for all $\triangle t>0$ and $0<\delta \leq 1 / 2$ satisfying the condition

$$
\tau^{4} \triangle t\left(\delta^{4} \min \left\{T^{3}, T^{6}\right\}\right)^{-1} \leq \varepsilon_{0},
$$

and $q$ is any time discrete function in $\left(H^{2}(\Omega) \cap H_{0}^{1}(\Omega)\right)^{\overline{\mathcal{D}}}$.

To prove the Carleman estimate, we proceed as close as possible to the continuous case and follow the procedure presented in [10]. During the proof, we will clarify the main differences and difficulties introduced by time discretization.

Remark 1.5. Some remarks are in order.

- One can readily recognize from (1.15) the classical structure of a Carleman inequality in the continuous setting (cf. [10, Lemma 1.3]). The last three terms are, however, specific to the discrete case and arise during the proof. In fact, the presence of these terms is important: otherwise we could obtain a classical observability inequality (not relaxed) leading to a uniform controllability result (w.r.t $\triangle t$ ) for (1.6), which would be a contradiction with the known results (see [22]).

Note that, despite the presence of a $(\triangle t)^{-1}$ factor, those three terms are actually exponentially small with respect to $\triangle t$ since $\varphi<0$ and $\theta$ is large (close to $\frac{1}{\delta T^{2}}$ ) near $t=0$ and $t=T$.

- As compared to other discrete (in space) Carleman results (see [6]), the last term corresponding to the gradient of the function q cannot be avoided. Actually, this term also appears during the proof of the estimate presented in [6, Theorem 1.3] but since the functions are discrete in the space variable, it is approximated as $\nabla q \approx C h^{-1} q$, where $h$ is the mesh step size. In our case, it is not clear how to remove this term and how to prove estimate (1.15) without the last term remains open. 
- Even though it does not explicitly appear in our Carleman estimate since it is hidden in the definition (1.13) of $\theta$, the parameter $\delta$ plays a key role in the proof. As mentioned before, the main interest is to avoid the singularity of the weight $\theta(t)$ at times $t=0$ and $t=T$ (these singularities, which correspond to the case $\delta=0$, are systematically exploited in the continuous setting, see e.g. [12], but are rather difficult to handle in the discrete framework). Here, by taking $\delta>0$, we enable two different things: one one hand, we can define continuously the weight outside the time interval [0,T], since functions on the dual mesh $\overline{\mathcal{D}}$ have one extra point lying outside this interval (see Figure 1 ). On the other, it allow us to estimate the derivative of $\theta$ (see Lemma B.4) and set a suitable change of variables (see eq. (2.1)), which is the starting point of the proof.

- If one considers instead of $L_{\mathcal{D}}$ the following forward-in-time operator

$$
\left(\tilde{L}_{\mathcal{D}} q\right)^{n}:=\left(\bar{D}_{t} q\right)^{n}-\Delta\left(\overline{\mathrm{t}}^{+} q\right)^{n}, \quad n \in \llbracket 1, M \rrbracket,
$$

for any $q \in\left(H^{2}(\Omega) \cap H_{0}^{1}(\Omega)\right)^{\overline{\mathcal{D}}}$, then a similar Carleman estimate can be obtained: it is just needed to replace all the $\overline{\mathrm{t}}^{-}$operators by $\overline{\mathrm{t}}^{+}$and the last term by

$$
\int_{\Omega}\left|\left(e^{\tau \theta \varphi} \nabla q\right)^{\frac{1}{2}}\right|^{2}
$$

- Using the tools in Appendix B, Theorem 1.4 can be easily adapted to discrete parabolic operators acting on primal variables. For instance, we can consider the forward-in-time parabolic operator defined as follows

$$
\left(L_{\mathcal{P}} y\right)^{n+\frac{1}{2}}=\left(D_{t} y\right)^{n+\frac{1}{2}}-\Delta\left(\mathrm{t}^{+} y\right)^{n+\frac{1}{2}}, \quad n \in \llbracket 0, M-1 \rrbracket,
$$

for all $y \in\left(H^{2}(\Omega) \cap H_{0}^{1}(\Omega)\right)^{\overline{\mathcal{P}}}$. Then, under the same conditions of Theorem 1.4, we can prove the following estimate

$$
\begin{aligned}
\tau^{-1} f \int_{Q} \mathrm{t}^{+} & \left(e^{2 \tau \theta \varphi} \theta^{-1}\right)\left(\left|D_{t} y\right|^{2}+\left|\Delta\left(\mathrm{t}^{+} y\right)\right|^{2}\right)+\tau f \int_{Q} \mathrm{t}^{+}\left(e^{2 \tau \theta \varphi} \theta\right)\left|\nabla\left(\mathrm{t}^{+} y\right)\right|^{2}+\tau^{3} \iint_{Q} \mathrm{t}^{+}\left(e^{2 \tau \theta \varphi} \theta^{3}\right)\left(\mathrm{t}^{+} y\right)^{2} \\
\leq & C\left(f \int_{Q}\left(\mathrm{t}^{+} e^{2 \tau \theta \varphi}\right)\left|L_{\mathcal{P}} y\right|^{2}+\tau^{3} \iint_{\mathcal{B} \times(0, T)} \mathrm{t}^{+}\left(e^{2 \tau \theta \varphi} \theta^{3}\right)\left(\mathrm{t}^{+} y\right)^{2}\right) \\
& +C(\triangle t)^{-1}\left(\int_{\Omega}\left|\left(e^{\tau \theta \varphi} y\right)^{0}\right|^{2}+\int_{\Omega}\left|\left(e^{\tau \theta \varphi} y\right)^{M}\right|^{2}+\int_{\Omega}\left|\left(e^{\tau \theta \varphi} \nabla y\right)^{0}\right|^{2}\right)
\end{aligned}
$$

for any time discrete function $y \in\left(H^{2}(\Omega) \cap H_{0}^{1}(\Omega)\right)^{\overline{\mathcal{P}}}$.

As in the previous remark, we can adapt the result to the associated backward-in-time operator

$$
\left(\tilde{L}_{\mathcal{P}} y\right)^{n+\frac{1}{2}}=-\left(D_{t} y\right)^{n+\frac{1}{2}}-\Delta\left(\mathrm{t}^{-} y\right)^{n+\frac{1}{2}}, \quad n \in \llbracket 0, M-1 \rrbracket .
$$

\subsubsection{Controllability results: the linear case}

By considering a standard implicit Euler scheme for the time variable, the time-discrete homogeneous heat equation with potential $a \in L_{\mathcal{P}}^{\infty}(Q)$ reads as follows

$$
\begin{cases}\frac{y^{n+1}-y^{n}}{\triangle t}-\Delta y^{n+1}+a^{n+1} y^{n+1}=0, & n \in \llbracket 0, M-1 \rrbracket, \\ y_{\mid \partial \Omega}^{n+1}=0, & n \in \llbracket 0, M-1 \rrbracket, \\ y^{0}=y_{0} . & \end{cases}
$$

With the notation introduced previously, we can rewrite system (1.17) as

$$
\left\{\begin{array}{l}
\left(D_{t} y\right)^{n+\frac{1}{2}}-\Delta\left(\mathrm{t}^{+} y\right)^{n+\frac{1}{2}}+\left(\mathrm{t}^{+} a y\right)^{n+\frac{1}{2}}=0, \quad n \in \llbracket 0, M-1 \rrbracket, \\
y^{0}=y_{0} .
\end{array}\right.
$$

where, for convenience, we shall not explicitly write the homogeneous Dirichlet boundary conditions in such compact formulas since we will not deal with other boundary conditions in this paper. 
Observe that the equation verified by $y$ is written on the dual grid $\mathcal{D}$. This motivates us to look for a time-discrete control $v$ which is naturally attached to this grid (cf. equation (1.6)). Thus, we will consider controlled systems of the form

$$
\left\{\begin{array}{l}
\left(D_{t} y\right)^{n+\frac{1}{2}}-\Delta\left(\mathrm{t}^{+} y\right)^{n+\frac{1}{2}}+\left(\mathrm{t}^{+} a y\right)^{n+\frac{1}{2}}=\mathbf{1}_{\omega} v^{n+\frac{1}{2}}, \quad n \in \llbracket 0, M-1 \rrbracket, \\
y^{0}=y_{0} .
\end{array}\right.
$$

Following the well-known Hilbert Uniqueness Method (see Proposition 3.3), we can build a control function by minimizing a quadratic functional defined for the solutions to the adjoint of (1.18), which in this case is given by

$$
\begin{cases}\frac{q^{n-\frac{1}{2}}-q^{n+\frac{1}{2}}}{\Delta t}-\Delta q^{n-\frac{1}{2}}+a^{n} q^{n-\frac{1}{2}}=0, & n \in \llbracket 1, M \rrbracket, \\ q_{\mid \partial \Omega}^{n-\frac{1}{2}}=0, & n \in \llbracket 1, M \rrbracket, \\ q^{M+\frac{1}{2}}=q_{T} . & \end{cases}
$$

System (1.19) is the proper adjoint system of (1.18). With our notation, we can rewrite (1.19) in a more compact way, namely,

$$
\left\{\begin{array}{l}
-\left(\bar{D}_{t} q\right)^{n}-\Delta\left(\overline{\mathrm{t}}^{-} q\right)^{n}+a^{n}\left(\overline{\mathrm{t}}^{-} q\right)^{n}=0, \quad n \in \llbracket 1, M \rrbracket, \\
q^{M+\frac{1}{2}}=q_{T} .
\end{array}\right.
$$

Applying the Carleman inequality (1.15) to (1.20) and after a series of steps, we will deduce an observability inequality of the form

$$
\left|q^{\frac{1}{2}}\right|_{L^{2}(\Omega)} \leq C_{o b s}\left(f \int_{\omega \times(0, T)}|q|^{2}+e^{-\frac{C_{2}}{(\Delta t)^{1 / 4}}}\left|q_{T}\right|_{H_{0}^{1}(\Omega)}^{2}\right)^{\frac{1}{2}},
$$

for some positive constants $C_{o b s}$ and $C_{2}$ only depending on $T, \omega$ and $\|a\|_{\infty}$.

As mentioned before, this inequality has an extra term in the right-hand side as compared with the similar estimate in the continuous setting (1.4). This extra term is exponentially small which is actually an improvement compared to the similar inequality proved in [7].

With this inequality we are able to prove that there exists a discrete control $v \in L_{\mathcal{D}}^{2}\left(0, T ; L^{2}(\omega)\right)$ with $\|v\|_{L_{\mathcal{D}}^{2}\left(0, T ; L^{2}(\omega)\right)} \leq C$, uniformly with respect to $\triangle t$, such that the associated solution of (1.18) satisfies

$$
\left|y^{M}\right|_{H^{-1}(\Omega)} \leq C \sqrt{\phi(\triangle t)}\left|y_{0}\right|_{L^{2}(\Omega)}
$$

where $\triangle t \mapsto \phi(\triangle t)$ is any given function of the discretization parameter such that

$$
\liminf _{\triangle t \rightarrow 0} \frac{\phi(\triangle t)}{e^{-C_{2} /(\triangle t)^{1 / 4}}}>0
$$

Actually, this means that we reach a small target $y^{M}$ whose size goes to zero as the time step $\triangle t \rightarrow 0$, at the prescribed rate $\sqrt{\phi(\triangle t)}$ with controls that remain uniformly bounded with respect to $\triangle t$. In practice, computing such a control can be done by a minimization algorithm for which one can choose the function $\phi$ in such a way that the error $\sqrt{\phi(\triangle t)}$ on the target is comparable to the actual accuracy of the discretization scheme. We refer to [2] for a more complete discussion on that point.

The precise result is given by the following theorem.

Theorem 1.6. Let us consider $T>0$ and a discretization parameter $\triangle t$ sufficiently small. Then, for any $y_{0} \in L^{2}(\Omega)$ and any function $\phi$ verifying $(1.23)$, there exists a time-discrete control $v \in L_{\mathcal{D}}^{2}\left(0, T ; L^{2}(\omega)\right)$ such that

$$
\|v\|_{L_{\mathcal{D}}^{2}\left(0, T ; L^{2}(\omega)\right)} \leq C\left|y_{0}\right|_{L^{2}(\Omega)}
$$

and such that the associated solution $y$ to (1.17) verifies (1.22), for a positive constant $C$ only depending on $\phi, T$ and $\|a\|_{\infty}$ as in (3.3). 
Observe that Theorem 1.6 only yields a controllability result in $H^{-1}$. This is due to the presence of the term $\left|q_{T}\right|_{H_{0}^{1}}$ in the estimate (1.21) which in turn comes from the Carleman inequality (1.15) and is closely related to the selection of the weight function (1.12) (see Remarks 1.5 and 2.1). Using classical parabolic regularity results, we can actually obtain a controllability result in a $L^{2}$-setting.

Theorem 1.7. Let us consider $T>0$ and a discretization parameter $\triangle t$ sufficiently small. Let $0<$ $\widetilde{C}_{2}<C_{2}$. Then, for any $y_{0} \in L^{2}(\Omega)$ and any function $\phi$ verifying

$$
\liminf _{\triangle t \rightarrow 0} \frac{\phi(\triangle t)}{e^{-\widetilde{C}_{2}(\Delta t)^{1 / 4}}>0}
$$

there exists a time-discrete control $v$ such that

$$
\|v\|_{L_{\mathcal{D}}^{2}\left(0, T ; L^{2}(\omega)\right)} \leq C\left|y_{0}\right|_{L^{2}(\Omega)}
$$

and the associated solution $y$ to (1.18) verifies

$$
\left|y^{M}\right|_{L^{2}(\Omega)} \leq C \sqrt{\phi(\triangle t)}\left|y_{0}\right|_{L^{2}(\Omega)},
$$

where the positive constant $C$ depends only on $\phi, T$ and $\|a\|_{\infty}$ as in (3.3).

The methodology to prove Theorem 1.7 is to split the time interval in two parts. In the first (large) subinterval, we choose $v$ such that $y$ satisfies (1.22). In the second one, we set $v=0$ and let evolve the uncontrolled system. Then, from an elliptic regularity result, we finally deduce (1.25).

\subsubsection{Applications to the semilinear case}

One of the main advantages of using Carleman estimates for proving controllability results is the possibility of addressing nonlinear problems. Unlike [7] or [22], where spectral properties of the equations are needed (therefore restricted to the linear case), here we will study the controllability of the following implicit Euler scheme

$$
\begin{cases}\frac{y^{n+1}-y^{n}}{\triangle t}-\Delta y^{n+1}+f\left(y^{n+1}\right)=\mathbf{1}_{\omega} v^{n+\frac{1}{2}}, & n \in \llbracket 0, M-1 \rrbracket, \\ y_{\mid \partial \Omega}^{n+1}=0, & n \in \llbracket 0, M-1 \rrbracket, \\ y^{0}=y_{0} . & \end{cases}
$$

where $f \in C^{1}(\mathbb{R})$ is a globally Lipschitz function with $f(0)=0$. The result is the following.

Theorem 1.8. Let us consider $T>0$ and assume that $\triangle t$ is small enough. Then, for any $y_{0} \in L^{2}(\Omega)$ and any function verifying (1.23), there exists a uniformly bounded time-discrete control $v \in L_{\mathcal{D}}^{2}\left(0, T ; L^{2}(\omega)\right)$ such that the associated solution $y$ to (1.26) verifies (1.22).

The proof of Theorem 1.8 follows other well-known controllability results for nonlinear systems (see, for instance, $[9,11,19])$. First, we prove the existence of a $\phi(\triangle t)$-null control for a linearized version of (1.26) and then, after a careful analysis on the dependence of the constants appearing in (1.21) and using a fixed point argument, we deduce the result for the nonlinear case.

\subsection{Outline}

The rest of the paper is organized as follows. In section 2 we present the proof of Theorem 1.4. We have divided the proof in several steps to ease the reading. Section 3 is divided in two parts: in the first one, by employing the Carleman estimate (1.15), we obtain a relaxed observability inequality and then we use it to deduce the $\phi(\Delta t)$-null controllability result stated in Theorem 1.6. In the second one, we prove the $L^{2}$-null controllability result presented in Theorem 1.7. We devote Section 4 to prove the nonlinear result enunciated in Theorem 1.8. Finally, in Section 5 we present additional results and a brief discussion on the applicability of the Carleman estimate (1.15) for handling control problems for coupled systems. 


\section{Discrete-in-time Carleman estimate}

In this section, we present the proof of Theorem 1.4. For the sake of clarity, we have divided the proof in several steps. The ideas presented here try to follow as close as possible the proofs presented in [10] and [12] for the continuous case.

As in other works devoted to Carleman estimates, we will keep track of the dependence of all constants with respect to the parameters $\lambda, \tau$ and $T$. In addition, due to the discrete nature of our problem, we will pay special attention in the dependence of the discrete parameters $\triangle t$ and $\delta$.

In order to ease the computations, we introduce the following instrumental functions

$$
\begin{aligned}
s(t)=\tau \theta(t), & \tau>0, \quad t \in(-\delta T, T+\delta T), \\
r(x, t)=e^{s(t) \varphi(x)}, \quad \rho(x, t)=(r(x, t))^{-1}, & x \in \bar{\Omega}, \quad t \in(-\delta T, T+\delta T) .
\end{aligned}
$$

We begin by assuming that $q \in\left(C^{2}(\bar{\Omega}) \cap H_{0}^{1}(\Omega)\right)^{\overline{\mathcal{D}}}$, since we may then extend the result to functions in $H^{2}(\Omega)$ by an usual density argument. We introduce the change of variables

$$
z^{n+\frac{1}{2}}=r\left(., t_{n+1 / 2}\right) q^{n+\frac{1}{2}}, \quad n \in \llbracket 0, M \rrbracket,
$$

and then we will look for the equation satisfied by $z$. From now on, we will simplify the notation in such formulas by simply writing $z=r q$ which implicitely means that the (continuous) weight function $r$ is evaluated on the same grid (primal or dual) and at the same time as the one attached to the discrete variables. This will not lead to any ambiguity.

Since $\psi \in C^{2}(\bar{\Omega})$ and $q \in C^{2}(\bar{\Omega})^{\overline{\mathcal{D}}}$, we take the partial derivative with respect to the variable $x_{i}$ to obtain

$$
\partial_{i} z=r \partial_{i} q+\tau \theta \lambda \phi \partial_{i} \psi z
$$

where we have used that

$$
\partial_{i} \varphi=\lambda \phi \partial_{i} \psi
$$

A further derivation yields

$$
\begin{aligned}
\partial_{i}\left(\partial_{i} z\right)= & r \partial_{i}^{2} q+\tau^{2} \theta^{2} \lambda^{2}\left(\partial_{i} \psi\right)^{2} \phi^{2} z+\tau \theta \lambda^{2}\left(\partial_{i} \psi\right)^{2} \phi z \\
& +2 \tau \theta \lambda \partial_{i} \psi \phi r \partial_{i} q+\tau \theta \lambda \partial_{i}^{2} \psi \phi z
\end{aligned}
$$

Replacing (2.2) on the fourth term in (2.3), we get

$$
\begin{aligned}
\partial_{i}\left(\partial_{i} z\right)= & r \partial_{i}^{2} q-\tau^{2} \theta^{2} \lambda^{2}\left(\partial_{i} \psi\right)^{2} \phi^{2} z+\tau \theta \lambda^{2}\left(\partial_{i} \psi\right)^{2} \phi z \\
& +2 \tau \theta \lambda \partial_{i} \psi \phi \partial_{i} z+\tau \theta \lambda \partial_{i}^{2} \psi \phi z
\end{aligned}
$$

Now, since $r(x, t)$ and $\theta(t)$ are continuously defined for $t \in(-\delta T, T+\delta T)$, we can use the translation operator (1.10) to write the following equality on the primal grid $\mathcal{P}$,

$$
\begin{aligned}
\Delta\left(\overline{\mathrm{t}}^{-} z\right)= & \left(\overline{\mathrm{t}}^{-} r\right) \Delta\left(\overline{\mathrm{t}}^{-} q\right)-\tau^{2}\left(\overline{\mathrm{t}}^{-} \theta^{2}\right) \lambda^{2}|\nabla \psi|^{2} \phi^{2}\left(\overline{\mathrm{t}}^{-} z\right)+\tau\left(\overline{\mathrm{t}}^{-} \theta\right) \lambda^{2}|\nabla \psi|^{2} \phi\left(\overline{\mathrm{t}}^{-} z\right) \\
& +2 \tau\left(\overline{\mathrm{t}}^{-} \theta\right) \lambda \phi \nabla \psi \cdot \nabla\left(\overline{\mathrm{t}}^{-} z\right)+\tau\left(\overline{\mathrm{t}}^{-} \theta\right) \lambda \Delta \psi \phi\left(\overline{\mathrm{t}}^{-} z\right) .
\end{aligned}
$$

On the other hand, from Lemma B.4, formula (B.1) and the change of variables (2.1) we easily obtain

$$
\begin{aligned}
\bar{D}_{t} z & =\left(\overline{\mathrm{t}}^{-} r\right)\left(\bar{D}_{t} q\right)+\left(\overline{\mathrm{t}}^{+} q\right)\left(\bar{D}_{t} r\right) \\
& =\left(\overline{\mathrm{t}}^{-} r\right)\left(\bar{D}_{t} q\right)+\left[\tau\left(\overline{\mathrm{t}}^{+} \theta^{\prime}\right) \phi+\Delta t\left(\frac{\tau}{\delta^{3} T^{4}}+\frac{\tau^{2}}{\delta^{4} T^{6}}\right) \mathcal{O}_{\lambda, \kappa}(1)\right]\left(\overline{\mathrm{t}}^{+} z\right) .
\end{aligned}
$$

Observe that, unlike the continuous case, two additional terms corresponding to the time derivative $\bar{D}_{t} r$ appear. This will translate later into additional terms appearing on the right-hand side of the Carleman inequality.

Now, putting together (2.4)-(2.5) and using (1.14), we have that $z$ verifies, on the primal grid $\mathcal{P}$, the equation

$$
\begin{aligned}
\left(\bar{D}_{t} z\right)+\Delta\left(\overline{\mathrm{t}}^{-} z\right)= & -\left(\overline{\mathrm{t}}^{-} r\right)\left(L_{D} q\right)-\tau^{2}\left(\overline{\mathrm{t}}^{-} \theta^{2}\right) \lambda^{2}|\nabla \psi|^{2} \phi^{2}\left(\overline{\mathrm{t}}^{-} z\right)+\tau\left(\overline{\mathrm{t}}^{-} \theta\right) \lambda^{2}|\nabla \psi|^{2} \phi\left(\overline{\mathrm{t}}^{-} z\right) \\
& +2 \tau\left(\overline{\mathrm{t}}^{-} \theta\right) \lambda \phi \nabla \psi \cdot \nabla\left(\overline{\mathrm{t}}^{-} z\right)+\tau\left(\overline{\mathrm{t}}^{-} \theta\right) \lambda \Delta \psi \phi\left(\overline{\mathrm{t}}^{-} z\right)+\tau\left(\overline{\mathrm{t}}^{+} \theta^{\prime}\right) \phi\left(\overline{\mathrm{t}}^{+} z\right) \\
& +\Delta t\left(\frac{\tau}{\delta^{3} T^{4}}+\frac{\tau^{2}}{\delta^{4} T^{6}}\right) \mathcal{O}_{\lambda, \kappa}(1)\left(\overline{\mathrm{t}}^{+} z\right) .
\end{aligned}
$$


Using the definition of the difference operator $\bar{D}_{t}$, we can express the second last term in the above equation as

$$
\tau\left(\overline{\mathrm{t}}^{+} \theta^{\prime}\right) \phi\left(\overline{\mathrm{t}}^{+} z\right)=\tau\left(\overline{\mathrm{t}}^{+} \theta^{\prime}\right) \phi\left(\overline{\mathrm{t}}^{-} z\right)+\tau \Delta t\left(\overline{\mathrm{t}}^{+} \theta^{\prime}\right) \phi \bar{D}_{t} z
$$

We conveniently rewrite $(2.6)-(2.7)$ as follows

$$
A z+B z=g
$$

where $A z=A_{1} z+A_{2} z+A_{3} z, B z=B_{1} z+B_{2} z+B_{3} z$ with

$$
\begin{aligned}
g= & -\left(\overline{\mathrm{t}}^{-} r\right) L_{D} q+\tau\left(\overline{\mathrm{t}}^{-} \theta\right) \lambda \Delta \psi \phi\left(\overline{\mathrm{t}}^{-} z\right)-\tau\left(\overline{\mathrm{t}}^{-} \theta\right) \lambda^{2}|\nabla \psi|^{2} \phi\left(\overline{\mathrm{t}}^{-} z\right) \\
& +\Delta t\left(\frac{\tau}{\delta^{3} T^{4}}+\frac{\tau^{2}}{\delta^{4} T^{6}}\right) \mathcal{O}_{\lambda, \kappa}(1)\left(\overline{\mathrm{t}}^{+} z\right)+\tau \Delta t\left(\overline{\mathrm{t}}^{+} \theta^{\prime}\right) \phi \bar{D}_{t} z
\end{aligned}
$$

and

$$
\begin{array}{ll}
A_{1} z=-2 \tau\left(\overline{\mathrm{t}}^{-} \theta\right) \lambda^{2}|\nabla \psi|^{2} \phi\left(\overline{\mathrm{t}}^{-} z\right), & A_{2} z=-2 \tau\left(\overline{\mathrm{t}}^{-} \theta\right) \lambda \phi \nabla \psi \cdot \nabla\left(\overline{\mathrm{t}}^{-} z\right), \quad A_{3}=\bar{D}_{t} z, \\
B_{1} z=\tau^{2}\left(\overline{\mathrm{t}}^{-} \theta\right)^{2} \lambda^{2}|\nabla \psi|^{2} \phi^{2}\left(\overline{\mathrm{t}}^{-} z\right), \quad B_{2} z=\Delta\left(\overline{\mathrm{t}}^{-} z\right), \quad B_{3} z=-\tau \phi\left(\overline{\mathrm{t}}^{+} \theta^{\prime}\right)\left(\overline{\mathrm{t}}^{-} z\right) .
\end{array}
$$

With the notation introduced in Section 1.3, we can take the $L^{2}$-norm in (2.8), which yields

$$
\|A z\|_{L_{\mathcal{P}}^{2}(Q)}^{2}+\|B z\|_{L_{\mathcal{P}}^{2}(Q)}^{2}+2(A z, B z)_{L_{\mathcal{P}}^{2}(Q)}=\|g\|_{L_{\mathcal{P}}^{2}(Q)}^{2} .
$$

The rest of the proof will be dedicated to estimate the term $(A z, B z)_{L_{\mathcal{P}}^{2}(Q)}$. For clarity, we have divided it in several steps. Developing the inner-product $(A z, B z)_{L_{\mathcal{P}}^{2}(Q)}$, we set $I_{i j}:=\left(A_{i} z, B_{j} z\right)_{L_{\mathcal{P}}^{2}(Q)}$.

\section{Step 1. Estimates that do not involve the discrete operations}

Here, we will obtain lower bounds for different terms that do not involve the time discrete derivative $\bar{D}_{t}$. They can be carried out as in the continuous case, that is with suitable integration by parts in space, with some minor modifications. For the sake of completeness, we include the computations.

Estimate of $I_{11}+I_{21}$. First, it is clear that

$$
I_{11}=-2 \tau^{3} \lambda^{4} \iint_{Q}\left(\overline{\mathrm{t}}^{-} \theta\right)^{3}|\nabla \psi|^{4} \phi^{3}\left(\overline{\mathrm{t}}^{-} z\right)^{2} .
$$

On the other hand, we have

$$
\begin{aligned}
I_{21}= & 3 \tau^{3} \lambda^{4} \iint_{Q}\left(\overline{\mathrm{t}}^{-} \theta\right)^{3}|\nabla \psi|^{4} \phi^{3}\left(\overline{\mathrm{t}}^{-} z\right)^{2}+\tau^{3} \lambda^{3} \iint_{Q}\left(\overline{\mathrm{t}}^{-} \theta\right)^{3}|\nabla \psi|^{2} \Delta \phi^{3} \psi\left(\overline{\mathrm{t}}^{-} z\right)^{2} \\
& +2 \tau^{3} \lambda^{3} \sum_{i, j=1}^{N} \iint_{Q}\left(\overline{\mathrm{t}}^{-} \theta\right)^{3} \partial_{i} \psi \partial_{i j} \psi \partial_{j} \psi \phi^{3}\left(\overline{\mathrm{t}}^{-} z\right)^{2} \\
:= & I_{21}^{(1)}+I_{21}^{(2)}+I_{21}^{(3)}
\end{aligned}
$$

where we have integrated by parts with respect to the space variable. Then, adding up $I_{11}$ and $I_{21}^{(1)}$ and taking into account the properties of the weight function $\psi$, it is not difficult to see that

$$
I_{11}+I_{21}^{(1)} \geq C \tau^{3} \lambda^{4} \iint_{Q}\left(\overline{\mathrm{t}}^{-} \theta\right)^{3} \phi^{3}\left(\overline{\mathrm{t}}^{-} z\right)^{2}-C \tau^{3} \lambda^{4} \iint_{\omega \times(0, T)}\left(\overline{\mathrm{t}}^{-} \theta\right)^{3} \phi^{3}\left(\overline{\mathrm{t}}^{-} z\right)^{2}
$$

Since $\psi \in C^{2}(\bar{\Omega})$, we can take the parameter $\lambda$ sufficiently large to absorb $I_{21}^{(2)}$ and $I_{21}^{(3)}$ in the above inequality. More precisely, we have

$$
I_{11}+I_{21} \geq C \tau^{3} \lambda^{4} \iint_{Q}\left(\overline{\mathrm{t}}^{-} \theta\right)^{3} \phi^{3}\left(\overline{\mathrm{t}}^{-} z\right)^{2}-C \tau^{3} \lambda^{4} \iint_{\omega \times(0, T)}\left(\overline{\mathrm{t}}^{-} \theta\right)^{3} \phi^{3}\left(\overline{\mathrm{t}}^{-} z\right)^{2}
$$

for any $\lambda \geq C$. 
Estimate of $I_{12}+I_{22}$. Integrating by parts with respect to the space variable we have

$$
\begin{aligned}
I_{12}= & 2 \tau \lambda^{2} \iint_{Q}\left(\overline{\mathrm{t}}^{-} \theta\right)|\nabla \psi|^{2} \phi\left|\nabla\left(\overline{\mathrm{t}}^{-} z\right)\right|^{2}+2 \tau \lambda^{3} \iint_{Q}\left(\overline{\mathrm{t}}^{-} \theta\right)|\nabla \psi|^{2} \phi \nabla \psi \cdot \nabla\left(\overline{\mathrm{t}}^{-} z\right)\left(\mathrm{t}^{+} z\right) \\
& +4 \tau \lambda^{3} \sum_{i, j=1}^{N} \iint_{Q}\left(\overline{\mathrm{t}}^{-} \theta\right) \partial_{i} \psi \partial_{i j} \psi \phi \partial_{i}\left(\overline{\mathrm{t}}^{-} z\right)\left(\overline{\mathrm{t}}^{-} z\right) \\
:= & I_{12}^{(1)}+I_{12}^{(2)}+I_{12}^{(3)} .
\end{aligned}
$$

We will keep the term $I_{12}^{(1)}$. For the other two terms, we can use Cauchy-Schwarz and Young inequalities, together with the properties of $\psi$, to obtain

$$
\begin{aligned}
I_{12} \geq & I_{12}^{(1)}-C \tau \lambda^{4} \iint_{Q}\left(\overline{\mathrm{t}}^{-} \theta\right) \phi\left(\overline{\mathrm{t}}^{-} z\right)^{2}-C \tau \iint_{Q}\left(\overline{\mathrm{t}}^{-} \theta\right) \phi\left|\nabla\left(\overline{\mathrm{t}}^{-} z\right)\right|^{2} \\
& -C \tau^{2} \lambda^{4} \iint_{Q}\left(\overline{\mathrm{t}}^{-} \theta\right)^{2} \phi\left(\mathrm{t}^{+} z\right)^{2}-C \lambda^{2} \iint_{Q}\left|\nabla\left(\overline{\mathrm{t}}^{-} z\right)\right|^{2} .
\end{aligned}
$$

Hence, taking $\tau \geq C T^{2}$, we get

$$
I_{12} \geq I_{12}^{(1)}-C \iint_{Q}\left(\tau\left(\overline{\mathrm{t}}^{-} \theta\right) \phi+\lambda^{2}\right)\left|\nabla\left(\overline{\mathrm{t}}^{-} z\right)\right|^{2}-C \tau^{2} \lambda^{4} \iint_{Q}\left(\overline{\mathrm{t}}^{-} \theta\right)^{2} \phi^{2}\left(\overline{\mathrm{t}}^{-} z\right)^{2}
$$

Now, let us estimate the term $I_{22}$. Integrating by parts, we obtain

$$
\begin{aligned}
I_{22}= & -2 \iint_{Q} \tau\left(\overline{\mathrm{t}}^{-} \theta\right) \lambda \phi \nabla \psi \cdot \nabla\left(\overline{\mathrm{t}}^{-} z\right) \Delta\left(\overline{\mathrm{t}}^{-} z\right) \\
= & -2 \tau \lambda \iint_{\partial \Omega \times(0, T)}\left(\overline{\mathrm{t}}^{-} \theta\right) \phi \frac{\partial \psi}{\partial n}\left|\frac{\partial\left(\overline{\mathrm{t}}^{-} z\right)}{\partial n}\right|^{2}+2 \tau \lambda^{2} \sum_{i, j=1}^{N} \iint_{Q}\left(\overline{\mathrm{t}}^{-} \theta\right) \phi \partial_{i j} \psi \partial_{i}\left(\overline{\mathrm{t}}^{-} z\right) \partial_{j}\left(\overline{\mathrm{t}}^{-} z\right) \\
& +2 \tau \lambda \iint_{Q}\left(\overline{\mathrm{t}}^{-} \theta\right) \phi\left|\nabla \psi \cdot \nabla\left(\overline{\mathrm{t}}^{-} z\right)\right|^{2}+\tau \lambda \iint_{Q}\left(\overline{\mathrm{t}}^{-} \theta\right) \phi \nabla \psi \cdot \nabla\left|\nabla\left(\overline{\mathrm{t}}^{-} z\right)\right|^{2},
\end{aligned}
$$

where we have used the fact that $\nabla\left(\overline{\mathrm{t}}^{-} z\right) \cdot n=\frac{\partial\left(\overline{\mathrm{t}}^{-} z\right)}{\partial n}$ since $z_{\mid \partial \Omega}^{n+\frac{1}{2}}=0$ for any $n \in \llbracket 0, M-1 \rrbracket$. Integrating by parts in the fourth term of the above expression yields

$$
\begin{aligned}
\tau \lambda \iint_{Q}\left(\overline{\mathrm{t}}^{-} \theta\right) \phi \nabla \psi \cdot \nabla\left|\nabla\left(\overline{\mathrm{t}}^{-} z\right)\right|^{2}= & \tau \lambda \iint_{\partial \Omega \times(0, T)}\left(\mathrm{t}^{+} \theta\right) \phi \frac{\partial \psi}{\partial n}\left|\frac{\partial\left(\overline{\mathrm{t}}^{-} z\right)}{\partial n}\right|^{2}-\tau \lambda \iint_{Q}\left(\overline{\mathrm{t}}^{-} \theta\right) \Delta \psi \phi\left|\nabla\left(\overline{\mathrm{t}}^{-} z\right)\right|^{2} \\
& -\tau \lambda^{2} \iint_{Q}\left(\overline{\mathrm{t}}^{-} \theta\right)|\nabla \psi|^{2} \phi\left|\nabla\left(\overline{\mathrm{t}}^{-} \theta\right)\right|^{2}
\end{aligned}
$$

Thus, putting together (2.14) and (2.15), we obtain

$$
\begin{aligned}
I_{22}= & -\tau \lambda \iint_{\partial \Omega \times(0, T)}\left(\overline{\mathrm{t}}^{-} \theta\right) \phi \frac{\partial \psi}{\partial n}\left|\frac{\partial\left(\overline{\mathrm{t}}^{-} z\right)}{\partial n}\right|^{2}+2 \tau \lambda^{2} \sum_{i, j=1}^{N} \iint_{Q}\left(\overline{\mathrm{t}}^{-} \theta\right) \phi \partial_{i j} \psi \partial_{i}\left(\overline{\mathrm{t}}^{-} z\right) \partial_{j}\left(\overline{\mathrm{t}}^{-} z\right) \\
& +2 \tau \lambda \iint_{Q}\left(\overline{\mathrm{t}}^{-} \theta\right) \phi\left|\nabla \psi \cdot \nabla\left(\overline{\mathrm{t}}^{-} z\right)\right|^{2}-\tau \lambda \iint_{Q}\left(\overline{\mathrm{t}}^{-} \theta\right) \Delta \psi \phi\left|\nabla\left(\overline{\mathrm{t}}^{-} z\right)\right|^{2}-\tau \lambda^{2} \iint_{Q}\left(\overline{\mathrm{t}}^{-} \theta\right)|\nabla \psi|^{2} \phi\left|\nabla\left(\overline{\mathrm{t}}^{-} \theta\right)\right|^{2} \\
:= & \sum_{j=1}^{5} I_{22}^{(j)}
\end{aligned}
$$

From this expression, it is clear that $I_{22}^{(3)}$ is a positive term. Moreover, from the properties of the weight $\psi$, we also have that $I_{22}^{(1)} \geq 0$. Since $\psi \in C^{2}(\bar{\Omega})$, we can bound from below the second and fourth terms in the above expression to obtain

$$
I_{22} \geq-\tau \lambda^{2} \iint_{Q}\left(\overline{\mathrm{t}}^{-} \theta\right)|\nabla \psi|^{2} \phi\left|\nabla\left(\overline{\mathrm{t}}^{-} \theta\right)\right|^{2}-C \tau \lambda \iint_{Q}\left(\overline{\mathrm{t}}^{-} \theta\right) \lambda \phi\left|\nabla\left(\overline{\mathrm{t}}^{-} z\right)\right|^{2} .
$$


Collecting estimates (2.13) and (2.16), and taking $\lambda$ large enough, we get

$$
\begin{aligned}
I_{12}+I_{22} \geq & C \tau \lambda^{2} \iint_{Q}\left(\overline{\mathrm{t}}^{-} \theta\right) \phi\left|\nabla\left(\overline{\mathrm{t}}^{-} z\right)\right|^{2}-C \tau \lambda^{2} \iint_{\omega \times(0, T)}\left(\overline{\mathrm{t}}^{-} \theta\right) \phi\left|\nabla\left(\overline{\mathrm{t}}^{-} z\right)\right|^{2} \\
& -C \tau^{2} \lambda^{4} \iint_{Q}\left(\overline{\mathrm{t}}^{-} \theta\right)^{2} \phi^{2}\left(\overline{\mathrm{t}}^{-} z\right)^{2} .
\end{aligned}
$$

Then, using estimates (2.12) and (2.17) in equation (2.11), our Carleman inequality reads momentarily as follows

$$
\begin{aligned}
& \iint_{Q}\left(\tau^{3} \lambda^{4}\left(\overline{\mathrm{t}}^{-} \theta\right)^{3} \phi^{3}\left(\overline{\mathrm{t}}^{-} z\right)^{2}+\tau \lambda^{2}\left(\overline{\mathrm{t}}^{-} \theta\right) \phi\left|\nabla\left(\overline{\mathrm{t}}^{-} z\right)\right|^{2}\right)+\|A z\|_{L_{\mathcal{P}}^{2}(Q)}^{2}+\|B z\|_{L_{\mathcal{P}}^{2}(Q)}^{2} \\
& \quad+I_{31}+I_{32}+\sum_{j=1}^{3} I_{j 3} \leq\|g\|_{L_{\mathcal{P}}^{2}(Q)}^{2}+C\left(\iint_{\omega_{0} \times(0, T)}\left(\tau^{3} \lambda^{4}\left(\overline{\mathrm{t}}^{-} \theta\right)^{3} \phi^{3}\left(\overline{\mathrm{t}}^{-} z\right)^{2}+\tau \lambda^{2}\left(\overline{\mathrm{t}}^{-} \theta\right) \phi\left|\nabla\left(\overline{\mathrm{t}}^{-} z\right)\right|^{2}\right)\right)
\end{aligned}
$$

for $\lambda \geq C$ and $\tau \geq C T^{2}$.

\section{Step 2. Estimates involving discrete computations}

In the second part of the proof, we will use the discrete calculus results presented in Appendix B. They will help us to handle the remainder terms that arise when integrating by parts with respect to the time variable.

Estimate of $I_{31}$. Using formula (B.2) we have

$$
\begin{aligned}
I_{31} & =\tau^{2} \lambda^{2} \iint_{Q}\left(\overline{\mathrm{t}}^{-} \theta\right)^{2} \phi^{2}|\nabla \psi|^{2}\left(\overline{\mathrm{t}}^{-} z\right) \bar{D}_{t} z \\
& =\frac{1}{2} \tau^{2} \lambda^{2} \iint_{Q}\left(\overline{\mathrm{t}}^{-} \theta\right)^{2} \phi^{2}|\nabla \psi|^{2} \bar{D}_{t}\left(|z|^{2}\right)-\frac{\triangle t}{2} \tau^{2} \lambda^{2} \iint_{Q}\left(\overline{\mathrm{t}}^{-} \theta\right)^{2} \phi^{2}|\nabla \psi|^{2}\left(\bar{D}_{t} z\right)^{2} \\
& :=I_{31}^{(1)}+I_{31}^{(2)} .
\end{aligned}
$$

Integrating by parts with respect to time in the first term of the above expression, that is using (B.7), we obtain

$$
\begin{aligned}
I_{31}^{(1)}= & -\frac{1}{2} \tau^{2} \lambda^{2} \int_{\Omega}\left(\theta^{\frac{1}{2}}\right)^{2} \phi^{2}|\nabla \psi|^{2}\left(z^{\frac{1}{2}}\right)^{2}+\frac{1}{2} \tau^{2} \lambda^{2} \int_{\Omega}\left(\theta^{M+\frac{1}{2}}\right)^{2} \phi^{2}|\nabla \psi|^{2}\left(z^{M+\frac{1}{2}}\right)^{2} \\
& +\frac{1}{2} \tau^{2} \lambda^{2} \iint_{Q}|\nabla \psi|^{2} \phi^{2} \bar{D}_{t}\left(\theta^{2}\right)\left(\overline{\mathrm{t}}^{+} z\right)^{2} .
\end{aligned}
$$

Using estimate (B.12) and shifting the indices with (B.4)-(B.5) in the last term of the above expression yields

$$
\begin{aligned}
\left.\left|\frac{1}{2} \tau^{2} \lambda^{2} \iint_{Q}\right| \nabla \psi\right|^{2} \phi^{2} \bar{D}_{t}\left(\theta^{2}\right)\left(\overline{\mathrm{t}}^{+} z\right)^{2} \mid \leq & C \tau^{2} \lambda^{2} \iint_{Q}|\nabla \psi|^{2} \phi^{2}\left[T\left(\overline{\mathrm{t}}^{+} \theta\right)^{3}+\frac{\triangle t}{T^{6} \delta^{4}}\right]\left(\overline{\mathrm{t}}^{+} z\right)^{2} \\
= & C \tau^{2} \lambda^{2} \iint_{Q}|\nabla \psi|^{2} \phi^{2}\left[T\left(\overline{\mathrm{t}}^{-} \theta\right)^{3}+\frac{\triangle t}{T^{6} \delta^{4}}\right]\left(\overline{\mathrm{t}}^{-} z\right)^{2} \\
& +C \tau^{2} \lambda^{2} \int_{\Omega}|\nabla \psi|^{2} \phi^{2}\left[T\left(\theta^{M+\frac{1}{2}}\right)^{3}+\frac{\triangle t}{T^{6} \delta^{4}}\right]\left(z^{M+\frac{1}{2}}\right)^{2} \\
& -C \tau^{2} \lambda^{2} \int_{\Omega}|\nabla \psi|^{2} \phi^{2}\left[T\left(\theta^{\frac{1}{2}}\right)^{3}+\frac{\triangle t}{T^{6} \delta^{4}}\right]\left(z^{\frac{1}{2}}\right)^{2} .
\end{aligned}
$$

Hence, using that $\psi \in C^{2}(\bar{\Omega})$, we get

$$
\begin{aligned}
I_{31} \geq & -C \tau^{2} \lambda^{2} T \iint_{Q} \phi^{2}\left(\overline{\mathrm{t}}^{-} \theta\right)^{3}\left(\overline{\mathrm{t}}^{-} z\right)^{2}-C \triangle t \tau^{2} \lambda^{2} \iint_{Q}\left(\overline{\mathrm{t}}^{-} \theta\right)^{2} \phi^{2}\left(\bar{D}_{t} z\right)^{2} \\
& -C \tau^{2} \lambda^{2} \iint_{Q} \phi^{2} \frac{\triangle t}{T^{6} \delta^{4}}\left(\overline{\mathrm{t}}^{-} z\right)^{2}-W_{1}
\end{aligned}
$$


where

$$
W_{1}:=C \tau^{2} \lambda^{2} \int_{\Omega}\left(\theta^{\frac{1}{2}}\right)^{2} \phi^{2}\left(z^{\frac{1}{2}}\right)^{2}+C \tau^{2} \lambda^{2} \int_{\Omega} \phi^{2}\left[T\left(\theta^{M+\frac{1}{2}}\right)^{3}+\frac{\triangle t}{T^{6} \delta^{4}}\right]\left(z^{M+\frac{1}{2}}\right)^{2} .
$$

Estimate of $I_{32}$. Integrating by parts in the space variable and using (B.3), we have

$$
I_{32}=-\frac{1}{2} \iint_{Q} \bar{D}_{t}\left(|\nabla z|^{2}\right)+\frac{1}{2} \Delta t \iint_{Q}\left(\bar{D}_{t} \nabla z\right)^{2}
$$

and then integrating in time

$$
\begin{aligned}
I_{32} & =\frac{1}{2}\left(\int_{\Omega}\left|\nabla z^{\frac{1}{2}}\right|^{2}-\int_{\Omega}\left|\nabla z^{M+\frac{1}{2}}\right|^{2}\right)+\frac{\triangle t}{2} \iint_{Q}\left|D_{t}(\nabla z)\right|^{2} \\
& \geq-\frac{1}{2} \int_{\Omega}\left|\nabla z^{M+\frac{1}{2}}\right|^{2}=: W_{2} .
\end{aligned}
$$

Remark 2.1. Observe that in the continuous case the term $I_{32}$ is equal to zero. Here, a new term depending on $\nabla z^{M+\frac{1}{2}}$ appears and is related to the special structure of the function $\theta$ which prevents that the exponential weight vanishes at $t=T$.

Estimate of $I_{13}$. A straightforward computation gives

$$
I_{13}=-2 \tau^{2} \lambda^{2} \iint_{Q}|\nabla \psi|^{2}\left(\overline{\mathrm{t}}^{-} \theta\right) \phi^{2}\left(\overline{\mathrm{t}}^{+} \theta^{\prime}\right)\left(\overline{\mathrm{t}}^{-} z\right)^{2}
$$

then using (B.13) and that $\psi \in C^{2}(\bar{\Omega})$, we readily obtain

$$
\left|I_{13}\right| \leq C\left(\tau^{2} \lambda^{2} T \iint_{Q} \phi^{2}\left(\overline{\mathrm{t}}^{-} \theta\right)^{3}\left(\overline{\mathrm{t}}^{-} z\right)^{2}+\tau^{2} \lambda^{2} \iint_{Q} \phi^{2} \frac{\triangle t}{T^{4} \delta^{3}}\left(\overline{\mathrm{t}}^{-} z\right)^{2}\right) .
$$

Estimate of $I_{23}$. Integration by parts in the space variable yields

$$
\begin{aligned}
I_{23} & =-2 \tau^{2} \lambda \iint_{Q}\left(\overline{\mathrm{t}}^{-} \theta\right) \phi^{2}\left(\overline{\mathrm{t}}^{+} \theta^{\prime}\right) \nabla \psi \cdot \nabla\left(\overline{\mathrm{t}}^{-} z\right)\left(\overline{\mathrm{t}}^{-} z\right) \\
& =\tau^{2} \lambda \iint_{Q}\left(\overline{\mathrm{t}}^{-} \theta\right)\left(\overline{\mathrm{t}}^{+} \theta^{\prime}\right) \phi^{2} \Delta \psi\left(\overline{\mathrm{t}}^{-} z\right)^{2}+2 \tau^{2} \lambda^{2} \iint_{Q}\left(\overline{\mathrm{t}}^{-} \theta\right)\left(\overline{\mathrm{t}}^{+} \theta^{\prime}\right) \phi^{2}|\nabla \psi|^{2}\left(\overline{\mathrm{t}}^{-} z\right)^{2} .
\end{aligned}
$$

From estimate (B.13) and since $\psi \in C^{2}(\bar{\Omega})$ is not difficult to see that for $\lambda \geq 1$ we have

$$
\left|I_{23}\right| \leq C\left(\tau^{2} \lambda^{2} T \iint_{Q}\left(\overline{\mathrm{t}}^{-} \theta\right)^{3} \phi^{2}\left(\overline{\mathrm{t}}^{-} z\right)^{2}+\tau^{2} \lambda^{2} \iint_{Q}\left(\overline{\mathrm{t}}^{-} \theta\right) \phi^{2} \frac{\Delta t}{T^{4} \delta^{3}}\left(\overline{\mathrm{t}}^{-} z\right)^{2}\right) .
$$

Estimate of $I_{33}$. Using formula (B.3) we can write

$$
\begin{aligned}
I_{33} & =-\tau \iint_{Q} \phi\left(\overline{\mathrm{t}}^{+} \theta^{\prime}\right) \bar{D}_{t} z\left(\overline{\mathrm{t}}^{-} z\right) \\
& =-\frac{1}{2} \tau \iint_{Q} \phi\left(\overline{\mathrm{t}}^{+} \theta^{\prime}\right) \bar{D}_{t}\left(z^{2}\right)+\frac{1}{2} \tau \Delta t \iint_{Q} \phi\left(\overline{\mathrm{t}}^{+} \theta^{\prime}\right)\left(\bar{D}_{t} z\right)^{2}
\end{aligned}
$$

and integrating by parts on the first term, with (B.7), we obtain

$$
\begin{aligned}
I_{33}= & \frac{1}{2} \tau \int_{\Omega} \phi\left(\theta^{\prime}\right)^{\frac{1}{2}}\left(z^{\frac{1}{2}}\right)^{2}-\frac{1}{2} \tau \int_{\Omega} \phi\left(\theta^{\prime}\right)^{M+\frac{1}{2}}\left(z^{M+\frac{1}{2}}\right)^{2}+\frac{1}{2} \tau \iint_{Q} \phi \bar{D}_{t}\left(\theta^{\prime}\right)\left(\overline{\mathrm{t}}^{-} z\right)^{2} \\
& +\frac{1}{2} \tau \triangle t \iint_{Q} \phi\left(\overline{\mathrm{t}}^{+} \theta^{\prime}\right)\left(\bar{D}_{t} z\right)^{2} .
\end{aligned}
$$

From this expression and the definition of $\theta$, it is clear that the first two terms are negative. Moreover, from (B.14) we observe that the third term is positive. A further computation using (B.13) yields

$$
I_{33} \geq-W_{3}-C T \tau \Delta t \iint_{Q} \phi\left(\overline{\mathrm{t}}^{-} \theta\right)^{2}\left(\bar{D}_{t} z\right)^{2}-C \frac{\tau(\triangle t)^{2}}{T^{4} \delta^{3}} \iint_{Q} \phi\left(\bar{D}_{t} z\right)^{2},
$$


where

$$
W_{3}:=\frac{1}{2} \tau \int_{\Omega} \phi\left(\theta^{\prime}\right)^{M+\frac{1}{2}}\left(z^{M+\frac{1}{2}}\right)^{2}-\frac{1}{2} \tau \int_{\Omega} \phi\left(\theta^{\prime}\right)^{\frac{1}{2}}\left(z^{\frac{1}{2}}\right)^{2}
$$

Conclusion of Step 2. We will use inequalities (2.19)-(2.23) to estimate the left-hand side of (2.18).

Notice that the first term of estimates (2.19), (2.21), and (2.22) are analogous to those appearing in the continuous case and they can be absorbed as soon as $\lambda \geq 1$ and $\tau \geq C T$. The other terms contain parameters related to the time discretization (i.e., $\triangle t$ or $\delta$ ) and will be handled in a later step. Momentarily, we have

$$
\begin{aligned}
\iint_{Q} & \left(\tau^{3} \lambda^{4}\left(\overline{\mathrm{t}}^{-} \theta\right)^{3} \phi^{3}\left(\overline{\mathrm{t}}^{-} z\right)^{2}+\tau \lambda^{2}\left(\overline{\mathrm{t}}^{-} \theta\right) \phi\left|\nabla\left(\overline{\mathrm{t}}^{-} z\right)\right|^{2}\right)+\|A z\|_{L_{\mathcal{P}}^{2}(Q)}^{2}+\|B z\|_{L_{\mathcal{P}}^{2}(Q)}^{2} \\
\leq & \|g\|_{L_{\mathcal{P}}^{2}(Q)}^{2}+C\left(\iint_{\omega_{0} \times(0, T)}\left(\tau^{3} \lambda^{4}\left(\overline{\mathrm{t}}^{-} \theta\right)^{3} \phi^{3}\left(\overline{\mathrm{t}}^{-} z\right)^{2}+\tau \lambda^{2}\left(\overline{\mathrm{t}}^{-} \theta\right) \phi\left|\nabla\left(\overline{\mathrm{t}}^{-} z\right)\right|^{2}\right)\right) \\
& +C(W+X+Y)
\end{aligned}
$$

for any $\lambda \geq C$ and $\tau \geq C\left(T+T^{2}\right)$ and where we have used the notation $W=W_{1}+W_{2}+W_{3}$ and

$$
\begin{aligned}
& X:=\tau^{2} \lambda^{2} \iint_{Q}\left(\overline{\mathrm{t}}^{-} \theta\right) \phi^{2} \frac{\triangle t}{T^{4} \delta^{3}}\left(\overline{\mathrm{t}}^{-} z\right)^{2}+\tau^{2} \lambda^{2} \iint_{Q} \phi^{2} \frac{\triangle t}{T^{6} \delta^{4}}\left(\overline{\mathrm{t}}^{-} z\right)^{2}+\tau^{2} \lambda^{2} \iint_{Q} \phi^{2} \frac{\triangle t}{T^{4} \delta^{3}}\left(\overline{\mathrm{t}}^{-} z\right)^{2}, \\
& Y:=\triangle t \tau^{2} \lambda^{2} \iint_{Q}\left(\overline{\mathrm{t}}^{-} \theta\right)^{2} \phi^{2}\left(\bar{D}_{t} z\right)^{2}+T \tau \triangle t \iint_{Q} \phi\left(\overline{\mathrm{t}}^{-} \theta\right)^{2}\left(\bar{D}_{t} z\right)^{2}+\frac{\tau(\triangle t)^{2}}{T^{4} \delta^{3}} \iint_{Q} \phi\left(\bar{D}_{t} z\right)^{2} .
\end{aligned}
$$

\section{Step 3. Adding $\bar{D}_{t} z$ and $\Delta\left(\overline{\mathrm{t}}^{-} z\right)$ in the left-hand side}

In this step, we will add integrals containing the terms $\left|\bar{D}_{t} z\right|^{2}$ and $\left|\Delta\left(\overline{\mathrm{t}}^{-} z\right)\right|^{2}$ to the left-hand side of (2.24). The former will help to absorb the terms in $Y$ (see eq. (2.26)) and the latter will be useful to elimate the local term of $\nabla\left(\overline{\mathrm{t}}^{-} z\right)$.

Using the equation verified by $A z$ (see eq. (2.10)), it is not difficult to see that

$$
\tau^{-1} \iint_{Q}\left(\overline{\mathrm{t}}^{-} \theta\right)^{-1} \phi^{-1}\left(\bar{D}_{t} z\right)^{2} \leq C\left(\|A z\|_{L_{\mathcal{P}}^{2}(Q)}^{2}+\tau \lambda^{4} \iint_{Q}\left(\overline{\mathrm{t}}^{-} \theta\right) \phi\left(\overline{\mathrm{t}}^{-} z\right)^{2}+\tau \lambda^{2} \iint_{Q}\left(\overline{\mathrm{t}}^{-} \theta\right) \phi\left|\nabla\left(\overline{\mathrm{t}}^{-} z\right)\right|^{2}\right)
$$

and, from (2.10) and (B.13), we deduce for $\tau \geq C T^{2}$

$$
\begin{aligned}
\tau^{-1} \iint_{Q}\left(\overline{\mathrm{t}}^{-} \theta\right)^{-1} \phi^{-1}\left|\Delta\left(\overline{\mathrm{t}}^{-} z\right)\right|^{2} \leq C( & \|B z\|_{L_{\mathcal{P}}^{2}(Q)}^{2}+\tau^{3} \lambda^{4} \iint_{Q}\left(\overline{\mathrm{t}}^{-} \theta\right)^{3} \phi^{3}\left(\overline{\mathrm{t}}^{-} z\right)^{2} \\
& \left.+\tau T^{2} \iint_{Q}\left(\overline{\mathrm{t}}^{-} \theta\right)^{3} \phi\left(\overline{\mathrm{t}}^{-} z\right)^{2}+\frac{(\triangle t)^{2} \tau^{2}}{T^{8} \delta^{6}} \iint_{Q} \phi\left(\overline{\mathrm{t}}^{-} z\right)^{2}\right) .
\end{aligned}
$$

Combining estimates (2.24) and (2.27)-(2.28), we can absorb the lower order terms to obtain

$$
\begin{aligned}
\tau^{-1} \iint_{Q}\left(\overline{\mathrm{t}}^{-} \theta\right)^{-1} \phi^{-1}\left[\left(\bar{D}_{t} z\right)^{2}+\left|\Delta\left(\overline{\mathrm{t}}^{-} z\right)\right|^{2}\right]+\tau^{3} \lambda^{4} \iint_{Q}\left(\overline{\mathrm{t}}^{-} \theta\right)^{3} \phi^{3}\left(\overline{\mathrm{t}}^{-} z\right)^{2}+\tau \lambda^{2} \iint_{Q}\left(\overline{\mathrm{t}}^{-} \theta\right) \phi\left|\nabla\left(\overline{\mathrm{t}}^{-} z\right)\right|^{2} \\
\leq\|g\|_{L_{\mathcal{P}}^{2}(Q)}^{2}+C\left(\iint_{\omega_{0} \times(0, T)}\left(\tau^{3} \lambda^{4}\left(\overline{\mathrm{t}}^{-} \theta\right)^{3} \phi^{3}\left(\overline{\mathrm{t}}^{-} z\right)^{2}+\tau \lambda^{2}\left(\overline{\mathrm{t}}^{-} \theta\right) \phi\left|\nabla\left(\overline{\mathrm{t}}^{-} z\right)\right|^{2}\right)\right) \\
\quad+C(W+\tilde{X}+Y)
\end{aligned}
$$

for $\lambda \geq 1$ and $\tau \geq C T^{2}$, with $\tilde{X}=X+\frac{(\Delta t)^{2} \tau^{2}}{T^{8} \delta^{6}} \iint_{Q} \phi\left(\overline{\mathrm{t}}^{-} z\right)^{2}$.

\section{Step 4. Local estimate of the $\nabla\left(\mathrm{t}^{+} z\right)$}

We are now ready to eliminate local term of $\nabla\left(\overline{\mathrm{t}}^{-} z\right)$ in (2.29). We will proceed as in [10]. To this end, consider a cut-off function $\eta=\eta(x)$, with

$$
\eta \in C_{c}^{2}(\omega), \quad \eta \equiv 1 \quad \text { in } \omega_{0}, \quad 0 \leq \eta \leq 1 .
$$


Then, one can readily see that

$$
\begin{aligned}
\tau \lambda^{2} \iint_{\omega_{0} \times(0, T)}\left(\overline{\mathrm{t}}^{-} \theta\right) \phi\left|\nabla\left(\overline{\mathrm{t}}^{-} z\right)\right|^{2} \leq & \tau \lambda^{2} \iint_{\omega \times(0, T)}\left(\overline{\mathrm{t}}^{-} \theta\right) \phi\left|\nabla\left(\overline{\mathrm{t}}^{-} z\right)\right|^{2} \eta \\
= & -\tau \lambda^{2} \iint_{\omega \times(0, T)}\left(\overline{\mathrm{t}}^{-} \theta\right) \phi \Delta\left(\overline{\mathrm{t}}^{-} z\right)\left(\overline{\mathrm{t}}^{-} z\right) \eta \\
& -\tau \lambda^{2} \iint_{\omega \times(0, T)}\left(\overline{\mathrm{t}}^{-} \theta\right) \phi \nabla \eta \cdot \nabla\left(\overline{\mathrm{t}}^{-} z\right)\left(\overline{\mathrm{t}}^{-} z\right) \\
& -\tau \lambda^{3} \iint_{\omega \times(0, T)}\left(\overline{\mathrm{t}}^{-} \theta\right) \phi \nabla \psi \cdot \nabla\left(\overline{\mathrm{t}}^{-} z\right)\left(\overline{\mathrm{t}}^{-} z\right),
\end{aligned}
$$

and whence, employing Cauchy-Schwarz and Young inequalities, we get

$$
\begin{aligned}
\tau \lambda^{2} \iint_{\omega_{0} \times(0, T)}\left(\overline{\mathrm{t}}^{-} \theta\right) \phi\left|\nabla\left(\overline{\mathrm{t}}^{-} z\right)\right|^{2} \leq \gamma \iint_{Q} \tau^{-1}\left(\mathrm{t}^{+} \theta\right)^{-1} \phi^{-1}\left|\Delta\left(\overline{\mathrm{t}}^{-} z\right)\right|^{2} \\
+C_{\gamma} \iint_{\omega \times(0, T)}\left(\tau^{3} \lambda^{4}\left(\overline{\mathrm{t}}^{-} \theta\right)^{3} \phi^{3}\left(\overline{\mathrm{t}}^{-} z\right)^{2}+\tau \lambda^{4}\left(\overline{\mathrm{t}}^{-} \theta\right) \phi\left(\overline{\mathrm{t}}^{-} z\right)^{2}\right)
\end{aligned}
$$

for $\gamma>0$ small enough and where we have used the fact that $\lambda \geq 1$. This estimate together with (2.29) yield

$$
\begin{gathered}
\tau^{-1} \iint_{Q}\left(\overline{\mathrm{t}}^{-} \theta\right)^{-1} \phi^{-1}\left[\left(\bar{D}_{t} z\right)^{2}+\left|\Delta\left(\overline{\mathrm{t}}^{-} z\right)\right|^{2}\right]+\tau^{3} \lambda^{4} \iint_{Q}\left(\overline{\mathrm{t}}^{-} \theta\right)^{3} \phi^{3}\left(\overline{\mathrm{t}}^{-} z\right)^{2}+\tau \lambda^{2} \iint_{Q}\left(\overline{\mathrm{t}}^{-} \theta\right) \phi\left|\nabla\left(\overline{\mathrm{t}}^{-} z\right)\right|^{2} \\
\leq\|g\|_{L_{\mathcal{P}}^{2}(Q)}^{2}+C\left(\tau^{3} \lambda^{4} \iint_{\omega \times(0, T)}\left(\overline{\mathrm{t}}^{-} \theta\right)^{3} \phi^{3}\left(\overline{\mathrm{t}}^{-} z\right)^{2}+W+\widetilde{X}+Y\right)
\end{gathered}
$$

for $\lambda \geq C$ and $\tau \geq C\left(T+T^{2}\right)$.

\section{Step 5. Estimate of $g$ and absorbing the remaining terms}

In this step, we will absorb the reminder terms in the right-hand side of (2.30). This will be achieved by selecting in a specific order the parameters involved in the Carleman inequality.

We recall that from Lemma B.4, the condition $\triangle t \tau\|\varphi\|_{\infty}\left(T^{3} \delta^{2}\right)^{-1} \leq \kappa$ should be fulfilled in order to take the time derivative of the Carleman weight. We will choose $\lambda_{0} \geq 1$ sufficiently large and set $\lambda=\lambda_{0}$ for the remainder of the proof. Hence, we may choose

$$
\triangle t \tau\left(T^{3} \delta^{2}\right)^{-1} \leq \varepsilon
$$

for some positive constant $\varepsilon=\varepsilon\left(\lambda_{0}\right)$. We have the following

Lemma 2.2. Provided condition (2.31) holds, we have

$$
\begin{aligned}
\|g\|_{L_{\mathcal{P}}^{2}(Q)}^{2} \leq & C_{\lambda_{0}}\left(\iint_{Q}\left(\overline{\mathrm{t}}^{-} r\right)^{2}\left|L_{\mathcal{D}} q\right|^{2}+\tau^{2} \iint_{Q}\left(\overline{\mathrm{t}}^{-} \theta\right)^{2}\left(\overline{\mathrm{t}}^{-} z\right)^{2}\right) \\
& +C_{\lambda_{0}}\left(\frac{(\triangle t)^{2} \tau^{2}}{\delta^{6} T^{8}}+\frac{(\triangle t)^{2} \tau^{4}}{\delta^{8} T^{12}}\right)\left(\iint_{Q}\left(\overline{\mathrm{t}}^{-} z\right)^{2}+\iint_{\Omega}\left(z^{M+\frac{1}{2}}\right)^{2}\right) \\
& +C_{\lambda_{0}}\left(\tau^{2}(\triangle t)^{2} T^{2} \iint_{Q}\left(\overline{\mathrm{t}}^{-} \theta\right)^{4}\left(\bar{D}_{t} z\right)^{2}+\frac{(\triangle t)^{4} \tau^{2}}{T^{8} \delta^{6}} \iint_{Q}\left(\bar{D}_{t} z\right)^{2}\right) .
\end{aligned}
$$

Lemma 2.2 follows from estimate (B.13) and a straightforward computation, so for brevity we omit the proof. Using (2.32) in the inequality (2.30), we deduce that the following inequality holds

$$
\begin{gathered}
\tau^{-1} \iint_{Q}\left(\overline{\mathrm{t}}^{-} \theta\right)^{-1}\left[\left(\bar{D}_{t} z\right)^{2}+\left|\Delta\left(\overline{\mathrm{t}}^{-} z\right)\right|^{2}\right]+\tau^{3} \iint_{Q}\left(\overline{\mathrm{t}}^{-} \theta\right)^{3}\left(\overline{\mathrm{t}}^{-} z\right)^{2}+\tau \iint_{Q}\left(\overline{\mathrm{t}}^{-} \theta\right)\left|\nabla\left(\overline{\mathrm{t}}^{-} z\right)\right|^{2} \\
\leq C_{\lambda_{0}}\left(\iint_{Q}\left(\overline{\mathrm{t}}^{-} r\right)^{2}\left|L_{D} q\right|^{2}+\tau^{3} \iint_{\omega \times(0, T)}\left(\overline{\mathrm{t}}^{-} \theta\right)^{3}\left(\overline{\mathrm{t}}^{-} z\right)^{2}+\underline{W}+\underline{X}+\underline{Y}\right),
\end{gathered}
$$


for any $\tau \geq \tau_{0}\left(T+T^{2}\right)$, where $\tau_{0}$ is a positive constant depending on $\lambda_{0}$ and where we have grouped similar terms with the expressions of $W, \widetilde{X}$ and $Y$ to obtain

$$
\begin{aligned}
\underline{W}= & \tau^{2} \int_{\Omega}\left(\theta^{\frac{1}{2}}\right)^{2}\left(z^{\frac{1}{2}}\right)^{2}+\tau^{2} \int_{\Omega}\left[T\left(\theta^{M+\frac{1}{2}}\right)^{3}+\frac{\triangle t}{T^{6} \delta^{4}}\right]\left(z^{M+\frac{1}{2}}\right)^{2}+\int_{\Omega}\left|\nabla z^{M+\frac{1}{2}}\right|^{2} \\
& +\tau \int_{\Omega}\left(\theta^{\prime}\right)^{M+\frac{1}{2}}\left(z^{M+\frac{1}{2}}\right)^{2}-\tau \int_{\Omega}\left(\theta^{\prime}\right)^{\frac{1}{2}}\left(z^{\frac{1}{2}}\right)^{2}+\left[\frac{(\triangle t)^{2} \tau^{2}}{\delta^{6} T^{8}}+\frac{(\triangle t)^{2} \tau^{4}}{\delta^{8} T^{12}}\right] \int_{\Omega}\left(z^{M+\frac{1}{2}}\right)^{2} \\
\underline{X}= & \tau^{2} \iint_{Q}\left(\overline{\mathrm{t}}^{-} \theta\right) \frac{\triangle t}{T^{4} \delta^{3}}\left(\overline{\mathrm{t}}^{-} z\right)^{2}+\tau^{2} \iint_{Q} \frac{\triangle t}{T^{6} \delta^{4}}\left(\overline{\mathrm{t}}^{-} z\right)^{2}+\tau^{2} \iint_{Q} \frac{\triangle t}{T^{4} \delta^{3}}\left(\overline{\mathrm{t}}^{-} z\right)^{2} \\
& +\frac{(\triangle t)^{2} \tau^{2}}{T^{8} \delta^{6}} \iint_{Q}\left(\overline{\mathrm{t}}^{-} z\right)^{2}+\frac{(\triangle t)^{2} \tau^{4}}{\delta^{8} T^{12}} \iint_{Q}\left(\overline{\mathrm{t}}^{-} z\right)^{2}, \\
\underline{Y}= & \triangle t \tau^{2} \iint_{Q}\left(\overline{\mathrm{t}}^{-} \theta\right)^{2}\left(\bar{D}_{t} z\right)^{2}+T \tau \triangle t \iint_{Q}\left(\overline{\mathrm{t}}^{-} \theta\right)^{2}\left(\bar{D}_{t} z\right)^{2}+\frac{\tau(\triangle t)^{2}}{T^{4} \delta^{3}} \iint_{Q}\left(\bar{D}_{t} z\right)^{2} \\
& +\tau^{2}(\triangle t)^{2} T^{2} \iint_{Q}\left(\overline{\mathrm{t}}^{-} \theta\right)^{4}\left(\bar{D}_{t} z\right)^{2}+\frac{(\triangle t)^{4} \tau^{2}}{T^{8} \delta^{6}} \iint_{Q}\left(\bar{D}_{t} z\right)^{2} .
\end{aligned}
$$

Notice that in the definitions of $\underline{X}$ and $\underline{Y}$ there are some terms containing powers of $\tau$ greater than their counterparts in the left-hand side of $(2.33)$ and thus preventing us from absorbing them directly. Using the discretization parameters $\delta$ and $\triangle t$ we will be able to do so. More precisely, we have

Lemma 2.3. For any $\lambda \geq 1$ and $\tau \geq 1$, there exists $\varepsilon=\varepsilon(\lambda)$ such that for $0<\triangle t \tau^{4}\left(\min \left\{T^{3}, T^{6}\right\} \delta^{4}\right)^{-1} \leq$ $\varepsilon$, the following estimate holds

$$
\underline{X}+\underline{Y} \leq \varepsilon\left(\tau^{3} \iint_{Q}\left(\overline{\mathrm{t}}^{-} \theta\right)^{3}\left(\overline{\mathrm{t}}^{-} z\right)^{2}\right)+\varepsilon\left(\tau^{-1} \iint_{Q}\left(\overline{\mathrm{t}}^{-} \theta\right)^{-1}\left(\bar{D}_{t} z\right)^{2}\right),
$$

for all $\tau \geq \tau_{1}\left(T+T^{2}\right)$.

We provide the proof of this result in Appendix A. By using Lemma 2.3 with $\varepsilon_{0}=1 / 2 C_{\lambda_{0}}$, where $C_{\lambda_{0}}$ is the constant appearing in (2.33), we can absorb all of the terms in $\underline{X}$ and $\underline{Y}$, whence

$$
\begin{gathered}
\tau^{-1} \iint_{Q}\left(\overline{\mathrm{t}}^{-} \theta\right)^{-1}\left[\left(\bar{D}_{t} z\right)^{2}+\left|\Delta\left(\overline{\mathrm{t}}^{-} z\right)\right|^{2}\right]+\tau^{3} \iint_{Q}\left(\overline{\mathrm{t}}^{-} \theta\right)^{3}\left(\overline{\mathrm{t}}^{-} z\right)^{2}+\tau \iint_{Q}\left(\overline{\mathrm{t}}^{-} \theta\right)\left|\nabla\left(\overline{\mathrm{t}}^{-} z\right)\right|^{2} \\
\leq C_{\lambda_{0}}\left(\iint_{Q}\left(\overline{\mathrm{t}}^{-} r\right)^{2}\left|L_{D} q\right|^{2}+\tau^{3} \iint_{\omega \times(0, T)}\left(\overline{\mathrm{t}}^{-} \theta\right)^{3}\left(\overline{\mathrm{t}}^{-} z\right)^{2}+\underline{W}\right) .
\end{gathered}
$$

As in other related works for the controllability of discretized systems (cf. [3, 4, 6]), the terms appearing in $\underline{W}$ cannot be removed and they can only be estimated. We have the following result

Lemma 2.4. For any $\lambda \geq 1$ and $\tau \geq 1$, there exists $\varepsilon=\varepsilon(\lambda)$ such that for $0<\Delta t \tau^{4}\left(\min \left\{T^{3}, T^{6}\right\} \delta^{4}\right)^{-1} \leq$ $\varepsilon$, the following estimate holds

$$
\underline{W} \leq \varepsilon(\triangle t)^{-1}\left(\int_{\Omega}\left(z^{\frac{1}{2}}\right)^{2}+\int_{\Omega}\left(z^{M+\frac{1}{2}}\right)^{2}+\int_{\Omega}\left|\nabla z^{M+\frac{1}{2}}\right|^{2}\right) .
$$

Proof. Under the hypothesis of the lemma and recalling that $\delta \leq 1 / 2$, we may deduce that $\triangle t \leq \delta T / 2$ and therefore

$$
\max _{t \in[0, T+\triangle t]} \theta(t) \leq \frac{2}{\delta T^{2}} \quad \text { and } \max _{t \in[0, T+\triangle t]}\left|\theta^{\prime}(t)\right| \leq \frac{2}{\delta^{2} T^{3}} .
$$

Then, inequality (2.36) follows from the above estimates and straightforward computations.

Combining (2.35) with (2.36) we obtain

$$
\begin{gathered}
\tau^{-1} \iint_{Q}\left(\overline{\mathrm{t}}^{-} \theta\right)^{-1}\left[\left(\bar{D}_{t} z\right)^{2}+\left|\Delta\left(\overline{\mathrm{t}}^{-} z\right)\right|^{2}\right]+\tau^{3} \iint_{Q}\left(\overline{\mathrm{t}}^{-} \theta\right)^{3}\left(\overline{\mathrm{t}}^{-} z\right)^{2}+\tau \iint_{Q}\left(\overline{\mathrm{t}}^{-} \theta\right)\left|\nabla\left(\overline{\mathrm{t}}^{-} z\right)\right|^{2} \\
\leq C_{\lambda_{0}}\left(\iint_{Q}\left(\overline{\mathrm{t}}^{-} r\right)^{2}\left|L_{\mathcal{D}} q\right|^{2}+\tau^{3} \iint_{\omega \times(0, T)}\left(\overline{\mathrm{t}}^{-} \theta\right)^{3}\left(\overline{\mathrm{t}}^{-} z\right)^{2}\right) \\
+(\triangle t)^{-1}\left(\int_{\Omega}\left(z^{\frac{1}{2}}\right)^{2}+\int_{\Omega}\left(z^{M+\frac{1}{2}}\right)^{2}+\int_{\Omega}\left|\nabla z^{M+\frac{1}{2}}\right|^{2}\right)
\end{gathered}
$$

for $\lambda \geq \lambda_{0}, \tau \geq \tau_{0}\left(T+T^{2}\right)$ and $\triangle t \tau^{4}\left(\min \left\{T^{3}, T^{6}\right\} \delta^{4}\right)^{-1} \leq \varepsilon_{0}$. 


\section{Step 7. Returning to the original variable and conclusion}

To conclude our proof, we will return to the original variable $y$. Recall that we have employed the change of variables $z=r q$. Thus, from (2.38), we readily obtain

$$
\begin{gathered}
\tau^{-1} \iint_{Q}\left(\overline{\mathrm{t}}^{-} \theta\right)^{-1}\left[\left(\bar{D}_{t} z\right)^{2}+\left|\Delta\left(\overline{\mathrm{t}}^{-} z\right)\right|^{2}\right]+\iint_{Q} \overline{\mathrm{t}}^{-}\left(r^{2} s^{3}\right)\left(\overline{\mathrm{t}}^{-} q\right)^{2}+\tau \iint_{Q}\left(\overline{\mathrm{t}}^{-} \theta\right)\left|\nabla\left(\overline{\mathrm{t}}^{-} z\right)\right|^{2} \\
\leq C_{\lambda_{0}}\left(\iint_{Q}\left(\overline{\mathrm{t}}^{-} r\right)^{2}\left|L_{\mathcal{D}} q\right|^{2}+\iint_{\omega \times(0, T)} \overline{\mathrm{t}}^{-}\left(r^{2} s^{3}\right)\left(\overline{\mathrm{t}}^{-} q\right)^{2}\right) \\
+(\triangle t)^{-1}\left(\int_{\Omega}\left|\left(e^{s \varphi} q\right)^{\frac{1}{2}}\right|^{2}+\int_{\Omega}\left|\left(e^{s \varphi} q\right)^{M+\frac{1}{2}}\right|^{2}+\int_{\Omega}\left|\left(e^{s \varphi} \nabla q\right)^{M+\frac{1}{2}}\right|^{2}\right)
\end{gathered}
$$

where we recall that $s(t)=\tau \theta(t)$ and where we have used formula (2.2) in the last term of (2.38). Using once again (2.2), it is not difficult to see that

$$
\iint_{Q} \overline{\mathrm{t}}^{-}\left(r^{2} s\right)\left|\nabla\left(\overline{\mathrm{t}}^{-} q\right)\right|^{2} \leq C_{\lambda_{0}} \iint_{Q}\left(\overline{\mathrm{t}}^{-} s\right)\left|\nabla\left(\overline{\mathrm{t}}^{-} z\right)\right|^{2}+C_{\lambda_{0}} \iint_{Q} \overline{\mathrm{t}}^{-}\left(r^{2} s^{3}\right)\left(\overline{\mathrm{t}}^{-} y\right)^{2} .
$$

Therefore, we can add the integral of $\left|\nabla\left(\overline{\mathrm{t}}^{-} q\right)\right|$ to the left-hand side of (2.39), that is

$$
\begin{aligned}
\tau^{-1} \iint_{Q}\left(\overline{\mathrm{t}}^{-} \theta\right)^{-1}\left[\left(\bar{D}_{t} z\right)^{2}+\left|\Delta\left(\overline{\mathrm{t}}^{-} z\right)\right|^{2}\right]+\iint_{Q} \overline{\mathrm{t}}^{-}\left(r^{2} s^{3}\right)\left(\overline{\mathrm{t}}^{-} q\right)^{2}+\iint_{Q} \overline{\mathrm{t}}^{-}\left(r^{2} s\right)\left|\nabla\left(\overline{\mathrm{t}}^{-} q\right)\right|^{2} \\
\leq C_{\lambda_{0}}\left(\iint_{Q}\left(\overline{\mathrm{t}}^{-} r\right)^{2}\left|L_{D} q\right|^{2}+\iint_{\omega \times(0, T)} \overline{\mathrm{t}}^{-}\left(r^{2} s^{3}\right)\left(\overline{\mathrm{t}}^{-} q\right)^{2}\right) \\
\quad+C_{\lambda_{0}}(\triangle t)^{-1}\left(\int_{\Omega}\left|\left(e^{s(t) \varphi} q\right)^{\frac{1}{2}}\right|^{2}+\int_{\Omega}\left|\left(e^{s(t) \varphi} q\right)^{M+\frac{1}{2}}\right|^{2}+\int_{\Omega}\left|\left(e^{s(t) \varphi} \nabla q\right)^{M+\frac{1}{2}}\right|^{2}\right) .
\end{aligned}
$$

Now, we use identity (2.3) and multiply both sides by $\overline{\mathrm{t}}^{-}\left(r^{2} s^{-1}\right) \Delta\left(\overline{\mathrm{t}}^{-} q\right)$. Integrating in $L^{2}(\Omega)$, summing over $n$, and using Hölder and Young inequalities yields

$$
\begin{aligned}
\iint_{Q} \overline{\mathrm{t}}^{-}\left(r^{2} s^{-1}\right)\left|\Delta\left(\overline{\mathrm{t}}^{-} q\right)\right|^{2} \leq C_{\lambda_{0}} & \left(\iint_{Q}\left(\overline{\mathrm{t}}^{-} s\right)^{-1}\left|\Delta\left(\overline{\mathrm{t}}^{-} z\right)\right|^{2}+\iint_{Q} \overline{\mathrm{t}}^{-}\left(r^{2} s\right)\left(\overline{\mathrm{t}}^{-} y\right)^{2}\right. \\
& \left.+\iint_{Q} \overline{\mathrm{t}}^{-}\left(r^{2} s\right)\left|\nabla\left(\overline{\mathrm{t}}^{-} y\right)\right|^{2}+\iint_{Q} \overline{\mathrm{t}}^{-}\left(r^{2} s^{3}\right)\left(\overline{\mathrm{t}}^{-} y\right)^{2}\right) .
\end{aligned}
$$

Since $\tau_{0}<\tau_{0}(1 / T+1) \leq \tau \theta(t)=s(t)$ for all $0 \leq t \leq T$, we obtain from the above expression

$$
\begin{aligned}
\iint_{Q} \overline{\mathrm{t}}^{-}\left(r^{2} s^{-1}\right)\left|\Delta\left(\overline{\mathrm{t}}^{-} q\right)\right|^{2} \leq C_{\lambda_{0}, \tau_{0}}( & \iint_{Q}\left(\overline{\mathrm{t}}^{-} s\right)^{-1}\left|\Delta\left(\overline{\mathrm{t}}^{-} z\right)\right|^{2}+\iint_{Q} \overline{\mathrm{t}}^{-}\left(r^{2} s^{3}\right)\left(\overline{\mathrm{t}}^{-} y\right)^{2} \\
& \left.+\iint_{Q} \overline{\mathrm{t}}^{-}\left(r^{2} s\right)\left|\nabla\left(\overline{\mathrm{t}}^{-} y\right)\right|^{2}\right),
\end{aligned}
$$

and, consequently, we can add the term containing $\left|\Delta\left(\overline{\mathrm{t}}^{-} q\right)\right|$ to the left-hand side of $(2.40)$, that is,

$$
\begin{gathered}
\tau^{-1} \iint_{Q}\left(\overline{\mathrm{t}}^{-} \theta\right)^{-1}\left(\bar{D}_{t} z\right)^{2}+\iint_{Q} \overline{\mathrm{t}}^{-}\left(r^{2} s^{-1}\right)\left|\Delta\left(\overline{\mathrm{t}}^{-} q\right)\right|^{2}+\iint_{Q} \overline{\mathrm{t}}^{-}\left(r^{2} s^{3}\right)\left(\overline{\mathrm{t}}^{-} q\right)^{2}+\iint_{Q} \overline{\mathrm{t}}^{-}\left(r^{2} s\right)\left|\nabla\left(\overline{\mathrm{t}}^{-} q\right)\right|^{2} \\
\leq C_{\lambda_{0}, \tau_{0}}\left(\iint_{Q}\left(\overline{\mathrm{t}}^{-} r\right)^{2}\left|L_{D} q\right|^{2}+\iint_{\omega \times(0, T)} \overline{\mathrm{t}}^{-}\left(r^{2} s^{3}\right)\left(\overline{\mathrm{t}}^{-} q\right)^{2}\right) \\
+C_{\lambda_{0}, \tau_{0}}(\triangle t)^{-1}\left(\int_{\Omega}\left|\left(e^{s(t) \varphi} q\right)^{\frac{1}{2}}\right|^{2}+\int_{\Omega}\left|\left(e^{s(t) \varphi} q\right)^{M+\frac{1}{2}}\right|^{2}+\int_{\Omega}\left|\left(e^{s(t) \varphi} \nabla q\right)^{M+\frac{1}{2}}\right|^{2}\right) .
\end{gathered}
$$

Finally, we add the integral corresponding to $\left|\bar{D}_{t} q\right|$ to the left-hand side of (2.41). This simply follows from the fact that $-\bar{D}_{t} q=f+\Delta\left(\overline{\mathrm{t}}^{-} q\right)$. Indeed, we have

$$
\iint_{Q} \overline{\mathrm{t}}^{-}\left(r^{2} s^{-1}\right)\left|\bar{D}_{t} q\right|^{2} \leq 2 \iint_{Q} \overline{\mathrm{t}}^{-}\left(r^{2} s^{-1}\right)|f|^{2}+2 \iint_{Q} \overline{\mathrm{t}}^{-}\left(r^{2} s^{-1}\right)\left|\Delta\left(\overline{\mathrm{t}}^{-} q\right)\right|^{2} .
$$


Increasing, if necessary, the value of $\tau_{0}$ so $(\tau \theta(t))^{-1} \leq 1$ and (2.42) yields

$$
\begin{gathered}
\iint_{Q} \overline{\mathrm{t}}^{-}\left(r^{2} s^{-1}\right)\left(\bar{D}_{t} q\right)^{2}+\iint_{Q} \overline{\mathrm{t}}^{-}\left(r^{2} s^{-1}\right)\left|\Delta\left(\overline{\mathrm{t}}^{-} q\right)\right|^{2}+\iint_{Q} \overline{\mathrm{t}}^{-}\left(r^{2} s^{3}\right)\left(\overline{\mathrm{t}}^{-} q\right)^{2}+\iint_{Q} \overline{\mathrm{t}}^{-}\left(r^{2} s\right)\left|\nabla\left(\overline{\mathrm{t}}^{-} q\right)\right|^{2} \\
\leq C_{\lambda_{0}, \tau_{0}}\left(\iint_{Q}\left(\overline{\mathrm{t}}^{-} r\right)^{2}\left|L_{D} q\right|^{2}+\iint_{\omega \times(0, T)} \overline{\mathrm{t}}^{-}\left(r^{2} s^{3}\right)\left(\overline{\mathrm{t}}^{-} q\right)^{2}\right) \\
\quad+C_{\lambda_{0}, \tau_{0}}(\triangle t)^{-1}\left(\int_{\Omega}\left|\left(e^{s \varphi} q\right)^{\frac{1}{2}}\right|^{2}+\int_{\Omega}\left|\left(e^{s \varphi} q\right)^{M+\frac{1}{2}}\right|^{2}+\int_{\Omega}\left|\left(e^{s \varphi} \nabla q\right)^{M+\frac{1}{2}}\right|^{2}\right)
\end{gathered}
$$

This concludes the proof.

\section{$3 \phi(\triangle t)$-null controllability: the linear case}

In this section, we will use the Carleman estimate (1.15) for deducing control properties of linear parabolic systems. First, we prove Theorem 1.6 which gives a controllability result where we reach a small target in $H^{-1}(\Omega)$. Then, using this result and some regularity estimates, we present the strategy to obtain a controllability result in the $L^{2}$-setting.

\subsection{Proof of Theorem $\mathbf{1 . 6}$}

Let us consider the following time-discrete parabolic problem with potential

$$
\left\{\begin{array}{l}
\left(D_{t} y\right)^{n+\frac{1}{2}}-\Delta\left(\mathrm{t}^{+} y\right)^{n+\frac{1}{2}}+\left(\mathrm{t}^{+} a y\right)^{n+\frac{1}{2}}=\mathbf{1}_{\omega} v^{n+\frac{1}{2}}, \quad n \in \llbracket 0, M-1 \rrbracket, \\
y^{0}=y_{0} .
\end{array}\right.
$$

To achieve a $\phi(\triangle t)$-controllability result for (3.1), we begin by proving a relaxed observability estimate for the solutions to the associated adjoint system, which in this case is given by

$$
\left\{\begin{array}{l}
-\left(\bar{D}_{t} q\right)^{n}-\Delta\left(\overline{\mathrm{t}}^{-} q\right)^{n}+a^{n}\left(\overline{\mathrm{t}}^{-} q\right)^{n}=0, \quad n \in \llbracket 1, M \rrbracket, \\
q^{M+\frac{1}{2}}=q_{T} .
\end{array}\right.
$$

We have the following observability estimate:

Proposition 3.1. There exist positive constants $C_{0}, C_{1}$ and $C_{2}$ such that for all $0<T<1$, all potential functions $a \in L_{\mathcal{P}}^{\infty}(Q)$ and $\triangle t \leq \min \left\{\widetilde{\triangle t},\left(4\|a\|_{\infty}\right)^{-1}\right\}$ where

$$
\widetilde{\triangle t}=C_{0}\left(T+T^{2}+T^{2}\|a\|_{\infty}^{2 / 3}\right)^{-3} T^{6}
$$

any solution to $(3.2)$ with $q_{T} \in H_{0}^{1}(\Omega)$ satisfies

$$
\left|q^{\frac{1}{2}}\right|_{L^{2}(\Omega)} \leq C_{o b s}\left(f \int_{\omega \times(0, T)}|q|^{2}+e^{-\frac{C_{2}}{(\Delta t)^{1 / 4}}}\left|\nabla q_{T}\right|_{L^{2}(\Omega)}^{2}\right)^{\frac{1}{2}}
$$

where

$$
C_{o b s}=e^{C_{1}\left(1+\frac{1}{T}+\|a\|_{\infty}^{2 / 3}+T\|a\|_{\infty}\right)} .
$$

Remark 3.2. Without loss of generality and since we are mostly interested in controllability in small time, we consider that $0<T<1$ so that we have $\min \left\{T^{3}, T^{6}\right\}=T^{6}$. For $T \geq 1$, the procedure can be adapted straightforwardly.

Proof. Applying the Carleman estimate (1.15) to the solutions of (3.2), we have that

$$
\begin{aligned}
\iint_{Q} \overline{\mathrm{t}}^{-}\left(e^{2 s \varphi} s^{3}\right)\left(\overline{\mathrm{t}}^{-} q\right)^{2} & \leq C\left(\iint_{Q}\left(\overline{\mathrm{t}}^{-} e^{2 s \varphi}\right)\left|a\left(\overline{\mathrm{t}}^{-} q\right)\right|^{2}+\iint_{\omega \times(0, T)} \overline{\mathrm{t}}^{-}\left(e^{2 s \varphi} s^{3}\right)\left(\overline{\mathrm{t}}^{-} q\right)^{2}\right) \\
& +C(\triangle t)^{-1}\left(\left|\left(e^{s \varphi} q\right)^{\frac{1}{2}}\right|_{L^{2}(\Omega)}^{2}+\left|\left(e^{s \varphi} q\right)^{M+\frac{1}{2}}\right|_{L^{2}(\Omega)}^{2}+\left|\left(e^{s \varphi} \nabla q\right)^{M+\frac{1}{2}}\right|_{L^{2}(\Omega)}^{2}\right)
\end{aligned}
$$


for all $\tau \geq \tau_{0}\left(T+T^{2}\right)$ and $\tau^{4} \triangle t\left(\delta^{4} T^{6}\right)^{-1} \leq \varepsilon_{0}$. Since $1 \leq C \theta T^{2}$, the first term in the right-hand side of (3.5) can be absorbed as soon as $\tau \geq C T^{2}\|a\|_{\infty}^{2 / 3}$, more precisely, we obtain

$$
\begin{aligned}
\iint_{Q} \overline{\mathrm{t}}^{-}\left(e^{2 s \varphi} s^{3}\right)\left(\overline{\mathrm{t}}^{-} q\right)^{2} & \leq C\left(\iint_{\omega \times(0, T)} \overline{\mathrm{t}}^{-}\left(e^{2 s \varphi} s^{3}\right)\left(\overline{\mathrm{t}}^{-} q\right)^{2}\right) \\
& +C(\triangle t)^{-1}\left(\left|\left(e^{s \varphi} q\right)^{\frac{1}{2}}\right|_{L^{2}(\Omega)}^{2}+\left|\left(e^{s \varphi} q\right)^{M+\frac{1}{2}}\right|_{L^{2}(\Omega)}^{2}+\left|\left(e^{s \varphi} \nabla q\right)^{M+\frac{1}{2}}\right|_{L^{2}(\Omega)}^{2}\right)
\end{aligned}
$$

for $\tau_{1} \geq \tau_{0}$ sufficiently large and

$$
\tau \geq \tau_{1}\left(T+T^{2}+T^{2}\|a\|_{\infty}^{2 / 3}\right)
$$

From (3.2), observe that $q^{n-\frac{1}{2}}$ solves the equation

$$
q^{n-\frac{1}{2}}-q^{n+\frac{1}{2}}-\triangle t \Delta q^{n-\frac{1}{2}}+\triangle t a^{n} q^{n-\frac{1}{2}}=0, \quad n \in \llbracket 1, M \rrbracket .
$$

Multiplying this expression by $q^{n-\frac{1}{2}}$ in $L^{2}(\Omega)$ and integrating by parts, we readily obtain

$$
\frac{1}{2}\left(\left|q^{n-\frac{1}{2}}\right|_{L^{2}(\Omega)}^{2}-\left|q^{n+\frac{1}{2}}\right|_{L^{2}(\Omega)}^{2}\right)+\frac{1}{2}\left|q^{n-\frac{1}{2}}-q^{n+\frac{1}{2}}\right|_{L^{2}(\Omega)}^{2}+\Delta t \int_{\Omega}\left|\nabla q^{n-\frac{1}{2}}\right|^{2}=-\Delta t \int_{\Omega} a^{n}\left|q^{n-\frac{1}{2}}\right|^{2} .
$$

A further computation gives, as soon as $2 \triangle t\|a\|_{\infty}<1$,

$$
\left|q^{n-\frac{1}{2}}\right|_{L^{2}(\Omega)}^{2} \leq \frac{1}{1-2 \Delta t\|a\|_{\infty}}\left|q^{n+\frac{1}{2}}\right|_{L^{2}(\Omega)}^{2}, \quad n \in \llbracket 1, M \rrbracket .
$$

Using the well-known inequality $e^{2 x}>1 /(1-x)$ for $0<x<1 / 2$ and from estimate (3.6), we deduce that

$$
\left|q^{\frac{1}{2}}\right|_{L^{2}(\Omega)}^{2} \leq e^{C T\|a\|_{\infty}}\left|q^{n+\frac{1}{2}}\right|_{L^{2}(\Omega)}^{2}, \quad n \in \llbracket 1, M \rrbracket,
$$

provided $\triangle t\|a\|_{\infty}<1 / 4$

Now, we will obtain a lower bound for the left-hand side of (3.5). Since we are adding positive terms, we can restrict to the indices $n \in \llbracket M / 4,3 M / 4 \rrbracket$ and hence

$$
\iint_{Q} \overline{\mathrm{t}}^{-}\left(e^{2 s \varphi} s^{3}\right)\left(\overline{\mathrm{t}}^{-} q\right)^{2} \geq \sum_{n \in \llbracket M / 4,3 M / 4 \rrbracket} \Delta t \tau^{3} \int_{\Omega}\left(e^{2 \tau \theta \varphi}\right)^{n-\frac{1}{2}}\left(\theta^{3}\right)^{n-\frac{1}{2}}\left|q^{n-\frac{1}{2}}\right|^{2} .
$$

Recalling that $\varphi$ is negative and independent of time, we deduce that

$$
\left(e^{2 \tau \theta \varphi}\right)^{n-\frac{1}{2}} \geq e^{-\frac{2^{5} \tau K_{0}}{15 T^{2}}}, \quad \forall n \in \llbracket M / 4,3 M / 4 \rrbracket,
$$

where $K_{0}:=\max _{x \in \bar{\Omega}}\{-\varphi(x)\}$. Moreover, since $\theta \geq 1 / T^{2}$ for all $t \in[0, T]$, we get

$$
\iint_{Q} \overline{\mathrm{t}}^{-}\left(e^{2 s \varphi} s^{3}\right)\left(\overline{\mathrm{t}}^{-} q\right)^{2} \geq \sum_{n \in \llbracket M / 4,3 M / 4 \rrbracket} \triangle t \tau^{3} e^{-\frac{2^{5} \tau K_{0}}{15 T^{2}}} T^{-6}\left|q^{n-\frac{1}{2}}\right|_{L^{2}(\Omega)}^{2} .
$$

Using estimate (3.7) in the above inequality and adding up, we get

$$
\begin{aligned}
\iint_{Q} \overline{\mathrm{t}}^{-}\left(e^{2 s \varphi} s^{3}\right)\left(\overline{\mathrm{t}}^{-} q\right)^{2} & \geq\left(\frac{T}{2}-\Delta t\right) \tau^{3} e^{-\frac{C \tau}{T^{2}}-C T\|a\|_{\infty}} T^{-6}\left|q^{\frac{1}{2}}\right|_{L^{2}(\Omega)}^{2} \\
& \geq C T e^{-\frac{C \tau}{T^{2}}-C T\|a\|_{\infty}}\left|q^{\frac{1}{2}}\right|_{L^{2}(\Omega)}^{2}
\end{aligned}
$$

for some $C>0$ only depending on $\Omega$ and $\omega$ and where we have used that $\tau \geq \tau_{1} T^{2}$.

From (2.37) and (3.7), we have that

$$
\begin{aligned}
\left|\left(e^{s \varphi} q\right)^{\frac{1}{2}}\right|_{L^{2}(\Omega)}^{2}+\left|\left(e^{s \varphi} q\right)^{M+\frac{1}{2}}\right|_{L^{2}(\Omega)}^{2} & \leq e^{-\frac{4 k_{0} \tau}{\delta T^{2}}}\left|q^{\frac{1}{2}}\right|^{2}+e^{-\frac{4 k_{0} \tau}{\delta T^{2}}}\left|q^{M+\frac{1}{2}}\right|^{2} \\
& \leq e^{-C \frac{\tau}{\delta T^{2}}+C T\|a\|_{\infty}}\left|q^{M+\frac{1}{2}}\right|^{2}
\end{aligned}
$$


where we have denoted $k_{0}:=\min _{x \in \bar{\Omega}}\{-\varphi(x)\}$. Using Poincaré inequality and the above estimate, we see that the last three terms in (3.5) can be bounded as

$$
\left|\left(e^{s \varphi} q\right)^{\frac{1}{2}}\right|_{L^{2}(\Omega)}^{2}+\left|\left(e^{s \varphi} q\right)^{M+\frac{1}{2}}\right|_{L^{2}(\Omega)}^{2}+\left|\left(e^{s \varphi} \nabla q\right)^{M+\frac{1}{2}}\right|_{L^{2}(\Omega)}^{2} \leq e^{-C \frac{\tau}{\delta T^{2}}+C T\|a\|_{\infty}}\left|\nabla q^{M+\frac{1}{2}}\right|^{2} .
$$

On the other hand, observe that the following estimate holds

$$
e^{2 s \varphi} s^{3} \leq \tau^{3} 2^{6} T^{-6} \exp \left(-\frac{2^{3} k_{0} \tau}{T^{2}}\right) \leq C, \quad \forall(x, t) \in Q,
$$

uniformly for $\tau \geq \frac{3}{8 k_{0}} T^{2}$. This, together with (3.9)-(3.10) can be used in (3.5) to obtain

$$
\left|q^{\frac{1}{2}}\right|_{L^{2}(\Omega)}^{2} \leq C T^{-1} e^{\frac{C \tau}{T^{2}}+C T\|a\|_{\infty}} \iint_{\omega \times(0, T)}\left(\overline{\mathrm{t}}^{-} q\right)^{2}+C(\triangle t)^{-1} e^{\frac{\tau}{T^{2}}\left(C-\frac{C}{\delta}\right)+C T\|a\|_{\infty}}\left|\nabla q^{M+\frac{1}{2}}\right|_{L^{2}(\Omega)}^{2}
$$

for any $\tau \geq \tau_{2}\left(T+T^{2}+T^{2}\|a\|_{\infty}^{2 / 3}\right)$ with $\tau_{2}=\max \left\{\tau_{1}, 3 / 8 k_{0}\right\}$. For $0<\delta \leq \delta_{1} \leq \delta_{0}$, with $\delta_{1}$ small enough, we obtain

$$
\left|q^{\frac{1}{2}}\right|_{L^{2}(\Omega)}^{2} \leq C T^{-1} e^{\frac{C \tau}{T^{2}}+C T\|a\|_{\infty}} \iint_{\omega \times(0, T)}\left(\overline{\mathrm{t}}^{-} q\right)^{2}+C(\triangle t)^{-1} e^{-\frac{C \tau}{\delta T^{2}}+C T\|a\|_{\infty}}\left|\nabla q^{M+\frac{1}{2}}\right|_{L^{2}(\Omega)}^{2} .
$$

To conclude the proof, we recall the condition of Theorem (1.4)

$$
\frac{\tau^{4} \triangle t}{\delta^{4} T^{6}} \leq \varepsilon_{0}
$$

which has to be fulfilled along $0<\delta \leq \delta_{1}$ and $\triangle t\|a\|_{\infty}<1 / 4$. Let us fix $\tau=\tau_{2}\left(T+T^{2}+T^{2}\|a\|^{2 / 3}\right)$ and define

$$
\widetilde{\triangle t}:=\frac{\varepsilon_{0}}{\tau_{2}^{4}} \delta_{1}^{4}\left(T+T^{2}+T^{2}\|a\|_{\infty}^{2 / 3}\right)^{-3} T^{6}
$$

whence

$$
\frac{\tau^{4} \widetilde{\triangle t}}{\delta_{1}^{4} T^{6}}=\varepsilon_{0}
$$

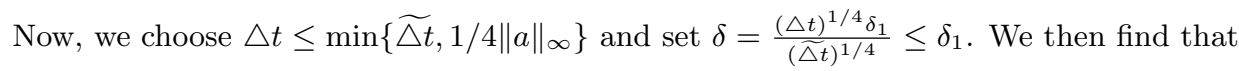

$$
\frac{\tau^{4} \triangle t}{\delta^{4} T^{6}}=\varepsilon_{0} .
$$

Therefore, $\tau /\left(T^{2} \delta\right)=\varepsilon^{1 / 4} T^{-1 / 2} /(\triangle t)^{1 / 4}$ and from (3.12) we deduce

$$
\left|q^{\frac{1}{2}}\right|_{L^{2}(\Omega)}^{2} \leq C T^{-1} e^{C\left(1+\frac{1}{T}+\|a\|_{\infty}^{2 / 3}+T\|a\|_{\infty}\right)} \iint_{\omega \times(0, T)}\left(\overline{\mathrm{t}}^{-} q\right)^{2}+e^{-\frac{C_{2}}{(\Delta t)^{1 / 4}}+C T\|a\|_{\infty}}\left|\nabla q^{M+\frac{1}{2}}\right|_{L^{2}(\Omega)}^{2} .
$$

Finally, shifting the indices in the integral term and grouping similar terms, we obtain

$$
\left|q^{\frac{1}{2}}\right|_{L^{2}(\Omega)}^{2} \leq e^{C_{1}\left(1+\frac{1}{T}+\|a\|_{\infty}^{2 / 3}+T\|a\|_{\infty}\right)}\left(\iint_{\omega \times(0, T)}|q|^{2}+e^{-\frac{C_{2}}{(\Delta t)^{1 / 4}}}\left|\nabla q^{M+\frac{1}{2}}\right|_{L^{2}(\Omega)}^{2}\right) .
$$

Thus, the proof is complete.

With the result of Proposition 3.1, we are in position to prove the following $\phi(\triangle t)$-null controllability result for (3.1).

Proposition 3.3. Let us consider $T>0$ and a discretization paramenter $\triangle t$ chosen as in Proposition 3.1. There exists a continuous and linear map $L_{T ; a}^{\Delta t}: L^{2}(\Omega) \rightarrow L_{\mathcal{D}}^{2}\left(0, T ; L^{2}(\omega)\right)$ such that for all initial data $y_{0} \in L^{2}(\Omega)$, there exists a time-discrete control function $v$ given by $v=L_{T ; a}^{\triangle t}\left(y_{0}\right)$ such that the solution to (3.1) satisfies

$$
\left|y^{M}\right|_{H^{-1}(\Omega)} \leq C_{o b s} \sqrt{\phi(\triangle t)}\left|y_{0}\right|_{L^{2}(\Omega)}
$$

and

$$
\|v\|_{L_{\mathcal{D}}^{2}\left(0, T ; L^{2}(\omega)\right)} \leq C_{o b s}\left|y_{0}\right|_{L^{2}(\Omega)}
$$

with $C_{\text {obs }}$ as given in Proposition 3.1. 
Proof. Consider the adjoint system (3.2). From the relaxed observability of Proposition 3.1 we have

$$
\left|q^{\frac{1}{2}}\right|_{L^{2}(\Omega)} \leq C_{o b s}\left(f \int_{\omega \times(0, T)}|q|^{2}+\phi(\triangle t)\left|q_{T}\right|_{H_{0}^{1}(\Omega)}^{2}\right)^{\frac{1}{2}},
$$

where we have chosen

$$
\phi(\triangle t)=e^{-\frac{C_{2}}{(\Delta t)^{1 / 4}}}
$$

We introduce the functional

$$
J_{\triangle t}\left(q_{T}\right)=\frac{1}{2} f \int_{\omega \times(0, T)}|q|^{2}+\frac{\phi(\triangle t)}{2}\left|q_{T}\right|_{H_{0}^{1}(\Omega)}^{2}+\left(y_{0}, q^{\frac{1}{2}}\right)_{L^{2}(\Omega)}, \quad \forall q_{T} \in H_{0}^{1}(\Omega)
$$

defined for the solutions to (3.2). It is clear that the functional $J$ is continuous and strictly convex. Moreover, using Hölder and Young inequality in the last term of the above expression, we have

$$
J_{\triangle t}\left(q_{T}\right) \geq \frac{1}{2} \iint_{\omega \times(0, T)}|q|^{2}+\frac{\phi(\triangle t)}{2}\left|q_{T}\right|_{H_{0}^{1}(\Omega)}^{2}-\frac{1}{4 C_{o b s}}\left|q^{\frac{1}{2}}\right|_{L^{2}(\Omega)}^{2}-C_{o b s}\left|y_{0}\right|_{L^{2}(\Omega)}^{2}
$$

Using inequality (3.13), we readily deduce that

$$
J_{\triangle t}\left(q_{T}\right) \geq \frac{1}{4} f \int_{\omega \times(0, T)}|q|^{2}+\frac{\phi(\triangle t)}{4}\left|q_{T}\right|_{H_{0}^{1}(\Omega)}^{2}-C_{o b s}\left|y_{0}\right|_{L^{2}(\Omega)}^{2}
$$

and therefore we can conclude that $J$ is coercive. This guarantees the existence of a unique minimizer that we denote $\widehat{q}_{T}$.

Now, consider $\widehat{q}$ the solution to (3.2) with initial datum $\widehat{q}_{T}$. The Euler-Lagrange equation associated with the minimization of functional (3.15) reads

$$
\iint_{\omega \times(0, T)} \widehat{q} q+\phi(\triangle t)\left(\nabla \widehat{q}_{T}, \nabla q_{T}\right)_{L^{2}(\Omega)}+\left(y_{0}, q^{\frac{1}{2}}\right)_{L^{2}(\Omega)}=0, \quad \forall q_{T} \in H_{0}^{1}(\Omega) .
$$

We set the control $v=L_{T ; a}^{\Delta t}\left(y_{0}\right)=\left(\mathbf{1}_{\omega} \widehat{q}^{n+\frac{1}{2}}\right)_{n \in \llbracket 0, M-1 \rrbracket}$ and consider the solution $y$ to the controlled problem

$$
\left\{\begin{array}{l}
\left(D_{t} y\right)^{n+\frac{1}{2}}-\Delta\left(\mathrm{t}^{+} y\right)^{n+\frac{1}{2}}+\left(\mathrm{t}^{+} a y\right)^{n+\frac{1}{2}}=\mathbf{1}_{\omega} \widehat{q}^{n+\frac{1}{2}} \quad n \in \llbracket 0, M-1 \rrbracket, \\
y^{0}=y_{0} .
\end{array}\right.
$$

By multiplying this equation by $q^{n+\frac{1}{2}}$ at each point of $\mathcal{D}$, and integrating by parts, we deduce

$$
\iint_{\omega \times(0, T)} \widehat{q} q=\left\langle y^{M}, q_{T}\right\rangle_{-1,1}-\left(y_{0}, q^{\frac{1}{2}}\right)_{L^{2}(\Omega)}
$$

where $\langle\cdot, \cdot\rangle_{-1,1}$ stands for the duality pairing between $H^{-1}(\Omega)$ and $H_{0}^{1}(\Omega)$. With $(3.16)$ and $(3.17)$, we conclude that

$$
y^{M}=\phi(\triangle t) \Delta \widehat{q}_{T}
$$

By taking $q_{T}=\widehat{q}_{T}$ in (3.16), we readily obtain

$$
\begin{aligned}
\|\widehat{q}\|_{L_{\mathcal{D}}^{2}\left(0, T ; L^{2}(\omega)\right)}^{2}+\phi(\Delta t)\left|\widehat{q}_{T}\right|_{H_{0}^{1}(\Omega)}^{2} & =-\left(y_{0}, \widehat{q}^{\frac{1}{2}}\right)_{L^{2}(\Omega)} \\
& \leq\left|y_{0}\right|_{L^{2}(\Omega)}\left|\widehat{q}^{\frac{1}{2}}\right|_{L^{2}(\Omega)}
\end{aligned}
$$

and from the observability inequality (3.13) we have

$$
\left|\widehat{q}^{\frac{1}{2}}\right|_{L^{2}(\Omega)}^{2} \leq C_{o b s}^{2}\left(f \int_{\omega \times(0, T)}|\widehat{q}|^{2}+\phi(\triangle t)\left|\widehat{q}_{T}\right|_{H_{0}^{1}(\Omega)}^{2}\right) .
$$

Combining the above expressions yields

$$
\|v\|_{L_{\mathcal{D}}^{2}\left(0, T ; L^{2}(\omega)\right)}=\|\widehat{q}\|_{L_{\mathcal{D}}^{2}\left(0, T ; L^{2}(\omega)\right)} \leq C_{o b s}\left|y_{0}\right|_{L^{2}(\Omega)}
$$


and

$$
\sqrt{\phi(\triangle t)}\left|\widehat{q}_{T}\right|_{H_{0}^{1}(\Omega)} \leq C_{o b s}\left|y_{0}\right|_{L^{2}(\Omega)}
$$

Hence, the linear map

$$
\begin{aligned}
L_{T ; a}^{\triangle t}: L^{2}(\Omega) & \rightarrow L_{\mathcal{D}}^{2}\left(0, T ; L^{2}(\omega)\right) \\
y_{0} & \mapsto v
\end{aligned}
$$

is well-defined and continuous. Finally, from the expressions (3.18)-(3.19) and the definition of the $H^{-1}$-norm, it is not difficult to see that

$$
\left|y^{M}\right|_{H^{-1}(\Omega)} \leq C_{o b s} \sqrt{\phi(\triangle t)}\left|y_{0}\right|_{L^{2}(\Omega)} .
$$

This finish the proof for $\phi$ as in (3.14). The claim immediately follows for any other function $\phi$ satisfying (1.23), changing the value of $C_{o b s}$ if necessary.

\subsection{Proof of Theorem 1.7}

The proof of Theorem 1.7 relies on Proposition 3.3. We will deduce that system (3.1) is $\phi(\triangle t)$-null controllable in $L^{2}(\Omega)$ by driving the solution first to a small target in $H^{-1}$ and then by letting the solution to evolve uncontrolled. In this way, by elliptic regularity, we will obtain a better estimate of the final target. Our strategy is as follows. Let $\phi$ satisfying (1.24) and let us set

$$
\tilde{\phi}(\triangle t)=\triangle t \phi(\triangle t)
$$

in such a way that $\tilde{\phi}$ satisfies (1.23).

Let us fix $T>0$, the initial data $y_{0} \in L^{2}(\Omega)$ and consider the time partition (1.5). We choose some (large) $T_{0}<T$ and set $M_{0}=\left\lfloor\frac{T_{0}}{\Delta t}\right\rfloor$. From Proposition 3.3, we know that there exists a time-discrete control $v_{0}=\left(v_{0}^{n+\frac{1}{2}}\right)_{n \in \llbracket 0, M_{0}-1 \rrbracket \text { with }}$

$$
\|v\|_{L_{\mathcal{D}}^{2}\left(0, T_{0} ; L^{2}(\omega)\right)} \leq C_{o b s}^{T_{0}}\left|y_{0}\right|_{L^{2}(\Omega)}
$$

such that $y$ solution to

$$
\begin{cases}\frac{y^{n+1}-y^{n}}{\triangle t}-\Delta y^{n+1}+a^{n+1} y^{n+1}=\mathbf{1}_{\omega} v_{0}^{n+\frac{1}{2}}, & n \in \llbracket 0, M_{0}-1 \rrbracket, \\ y_{\mid \partial \Omega}^{n+1}=0, & n \in \llbracket 0, M_{0}-1 \rrbracket, \\ y^{0}=y_{0}, & \end{cases}
$$

verifies

$$
\left|y^{M_{0}}\right|_{H^{-1}(\Omega)} \leq C_{o b s}^{T_{0}} \sqrt{\tilde{\phi}(\triangle t)}\left|y_{0}\right|_{L^{2}(\Omega)}
$$

where $C_{\text {obs }}^{T_{0}}$ is the observability constant corresponding to the time interval $\left(0, T_{0}\right)$. This defines the state $y^{n}$ for all $n \in \llbracket 0, M_{0} \rrbracket$.

Now, we set $v^{n+\frac{1}{2}}=0$ for $n \in \llbracket M_{0}, M-1 \rrbracket$ and consider the uncontrolled system

$$
\begin{cases}\frac{y^{n+1}-y^{n}}{\triangle t}-\Delta y^{n+1}+a^{n+1} y^{n+1}=0, & n \in \llbracket M_{0}, M-1 \rrbracket, \\ y_{\mid \partial \Omega}^{n+1}=0, & n \in \llbracket M_{0}, M-1 \rrbracket,\end{cases}
$$

with initial data $y^{M_{0}}$ coming from the sequence (3.20). Observe that for $n=M_{0}$, the equation verified for $y^{M_{0}+1}$ is given by

$$
-\Delta t \Delta y^{M_{0}+1}+\left(1+a^{M_{0}+1}\right) y^{M_{0}+1}=y^{n}, \quad \text { in } \Omega,
$$

from which we obtained the classical elliptic energy estimate

$$
-\sqrt{\triangle t}\left|y^{M_{0}+1}\right|_{H_{0}^{1}(\Omega)} \leq C\left(\Omega,\|a\|_{\infty}\right)\left|y^{M_{0}}\right|_{H^{-1}(\Omega)}
$$


This, together with estimate (3.21) and Poincaré's inequality yields

$$
\left|y^{M_{0}+1}\right|_{L^{2}(\Omega)} \leq C \sqrt{\frac{\tilde{\phi}(\triangle t)}{\triangle t}}\left|y_{0}\right|_{L^{2}(\Omega)},
$$

for some $C>0$ only depending on $\Omega, T$ and $\|a\|_{\infty}$. Arguing as in the proof of Proposition 3.1, we can iterate for indices $n \in \llbracket M_{0}+1, M \rrbracket$ to deduce that

$$
\left|y^{M}\right|_{L^{2}(\Omega)} \leq C \sqrt{\phi(\triangle t)}\left|y_{0}\right|_{L^{2}(\Omega)},
$$

where we have used the definition of $\tilde{\phi}$.

Therefore, we have constructed a sequence $y=\left\{y^{n}\right\}_{n \in \llbracket 0, M \rrbracket}$ by means of the auxiliary problems (3.20) and (3.22) such that $y^{M}$ verifies a $\phi(\triangle t)$-null controllability constraint in $L^{2}(\Omega)$. This concludes the proof.

\section{The nonlinear case}

We devote this section to prove the existence of controls for the semi-linear scheme (1.26). The proof follows well-known results for the controllability of semi-linear systems (see, for instance, [9, 11]) with some particularities due to the discrete nature of the problem.

Let us define

$$
g(s):= \begin{cases}\frac{f(s)}{s} & \text { if } s \neq 0, \\ f^{\prime}(0) & \text { if } s=0 .\end{cases}
$$

The assumptions on $f$ guarantees that $g$ and $f^{\prime}$ are well defined, continuous and bounded functions. For $\zeta \in L_{\mathcal{P}}^{2}(Q)$, we consider the linear system

$$
\begin{cases}\frac{y^{n+1}-y^{n}}{\triangle t}-\Delta y^{n+1}+g\left(\zeta^{n+1}\right) y^{n+1}=\mathbf{1}_{\omega} v^{n+\frac{1}{2}}, & n \in \llbracket 0, M-1 \rrbracket, \\ y_{\mid \partial \Omega}^{n+1}=0, & n \in \llbracket 0, M-1 \rrbracket, \\ y^{0}=y_{0} . & \end{cases}
$$

We set $a_{\zeta}^{n}=g\left(\zeta^{n}\right)$, so that we have

$$
\left\|a_{\zeta}\right\|_{\infty} \leq K, \quad \forall \zeta \in L_{\mathcal{D}}^{2}(Q),
$$

where $K$ is the Lipschitz constant of $f$.

In view of Propositions 3.1 and 3.3 , for $\triangle t$ chosen sufficiently small, i.e.,

$$
\triangle t \leq \min \left(\widetilde{\triangle t},(4 K)^{-1}\right)
$$

with

$$
\widetilde{\triangle t}=C\left(T+T^{2}+T^{2} K^{2 / 3}\right)^{-3} T^{6}
$$

we can build a control $v_{\zeta}=L_{T ; a_{\zeta}}^{\triangle t}\left(y_{0}\right)$ and the associated controlled solution to (4.1) such that

$$
\left|y_{\zeta}^{M}\right|_{L^{2}(\Omega)} \leq C e^{-\frac{C_{2}}{(\Delta t)^{-1 / 4}}}\left|y_{0}\right|_{L^{2}(\Omega)}, \quad\left\|v_{\zeta}\right\|_{L_{\mathcal{D}}^{2}\left(0, T ; L^{2}(\omega)\right)} \leq C\left|y_{0}\right|_{L^{2}(\Omega)}
$$

where $C_{1}>0$ and $C=\exp \left[C\left(1+\frac{1}{T}+K^{2 / 3}+T K\right)\right]$ are uniform with respect to $\zeta$ and the discretization parameter $\triangle t$. Notice that by selecting the parameter $\Delta t$ as in (4.3) guarantees on one hand the existence of a solution to (4.1) and the stability of the discrete scheme, while on the other we ensure the uniformity with respect to $\zeta$ in the estimates (4.4) which is important in what follows.

Let us define the map

$$
\begin{aligned}
\Lambda: L_{\mathcal{P}}^{2}(Q) & \rightarrow L_{\mathcal{P}}^{2}(Q) \\
\zeta & \mapsto y_{\zeta}
\end{aligned}
$$


where $y_{\zeta}$ is the solution to (4.1) associated to $a_{\zeta}^{n}=g\left(\zeta^{n}\right), n \in \llbracket 1, M \rrbracket$, and control as in (4.4). Arguing as in the proof of Proposition 3.1, we can readily deduce the energy estimate

$$
\left\|y_{\zeta}\right\|_{L_{\mathcal{P}}^{2}(Q)} \leq e^{C T\left\|a_{\zeta}\right\|_{\infty}}\left\|v_{\zeta}\right\|_{L_{\mathcal{D}}^{2}\left(0, T ; L^{2}(\omega)\right)} .
$$

Taking into account (4.2)-(4.5), we deduce that the image of $\Lambda$ is bounded, implying that there exists a closed convex set in $L_{\mathcal{P}}^{2}(Q)$ which is fixed by $\Lambda$. Moreover, it can be easily verified that $\Lambda$ is a continuous map from $L_{\mathcal{P}}^{2}(Q)$ into itself, while the uniform estimate

$$
\left\|y_{\zeta}\right\|_{L_{\mathcal{P}}^{2}\left(0, T ; H_{0}^{1}(\Omega)\right)} \leq C^{\prime}\left|y_{0}\right|_{L^{2}(\Omega)}
$$

for the solutions to (4.1) allows to conclude that $\Lambda$ is a compact map since $H_{0}^{1}(\Omega) \hookrightarrow L^{2}(\Omega)$ with compact embedding

All of the previous properties allow us to to apply Schauder fixed point theorem to deduce that there exists $y \in L_{\mathcal{P}}^{2}(Q)$ such that $\Lambda(y)=y$. Setting $v=L_{T ; a_{y}}^{\triangle t}\left(y_{0}\right)$ we obtain

$$
\begin{cases}\frac{y^{n+1}-y^{n}}{\triangle t}-\Delta y^{n+1}+f\left(y^{n+1}\right)=\mathbf{1}_{\omega} v^{n+\frac{1}{2}}, & n \in \llbracket 0, M-1 \rrbracket, \\ y_{\mid \partial \Omega}^{n+1}=0, & n \in \llbracket 0, M-1 \rrbracket, \\ y^{0}=y_{0}, & \end{cases}
$$

which concludes the proof as we have found a control $v$ that drives the solution of the semilinear semiimplicit parabolic system to a final state $y^{M}$ satisfying estimates (4.4).

\section{Applications to controllability of coupled systems}

Carleman estimate (1.15) can be used to study other controllability problems. We devote this section to study the controllability problem for a kind of coupled parabolic system. More precisely, we consider the $2 \times 2$ system

$$
\begin{cases}\frac{y_{1}^{n+1}-y_{1}^{n}}{\triangle t}-\Delta y_{1}^{n+1}+a_{11}^{n+1} y_{1}^{n+1}+a_{12}^{n+1} y_{2}^{n+1}=\mathbf{1}_{\omega} v^{n+\frac{1}{2}}, & n \in \llbracket 0, M-1 \rrbracket \\ \frac{y_{2}^{n+1}-y_{2}^{n}}{\triangle t}-\Delta y_{2}^{n+1}+a_{21}^{n+1} y_{1}^{n+1}+a_{22}^{n+1} y_{2}^{n+1}=0, & n \in \llbracket 0, M-1 \rrbracket \\ \left(y_{1}^{n+1}\right)_{\mid \partial \Omega}=\left(y_{2}^{n+1}\right)_{\mid \partial \Omega}=0, & n \in \llbracket 0, M-1 \rrbracket \\ y_{1}^{0}=y_{1,0}, \quad y_{2}^{0}=y_{2,0}, & \end{cases}
$$

for given initial data $y_{1,0}$ and $y_{2,0}$. with coupling coefficients $a_{i, j} \in L_{\mathcal{P}}^{\infty}(Q)$.

The idea here is to steer the solution to zero by acting only on the first equation of the system. As before, the control problem can be reduced to prove an observability inequality for the adjoint system

$$
\begin{cases}\frac{q_{1}^{n-\frac{1}{2}}-q_{1}^{n+\frac{1}{2}}}{\Delta t}-\Delta q_{1}^{n-\frac{1}{2}}+a_{11}^{n} q_{1}^{n-\frac{1}{2}}+a_{21}^{n} q_{2}^{n-\frac{1}{2}}=0, & n \in \llbracket 1, M \rrbracket, \\ \frac{q_{2}^{n-\frac{1}{2}}-q_{2}^{n+\frac{1}{2}}}{\Delta t}-\Delta q_{2}^{n-\frac{1}{2}}+a_{12}^{n} q_{1}^{n-\frac{1}{2}}+a_{22}^{n} q_{2}^{n-\frac{1}{2}}=0, & n \in \llbracket 1, M \rrbracket, \\ \left(q_{1}^{n-\frac{1}{2}}\right)_{\mid \partial \Omega}=\left(q_{2}^{n-1 \frac{1}{2}}\right)_{\mid \partial \Omega}=0, & n \in \llbracket 1, M \rrbracket, \\ q_{1}^{M+\frac{1}{2}}=q_{1, T}, \quad q_{2}^{M+\frac{1}{2}}=q_{2, T} . & \end{cases}
$$

This can be done by employing (1.15) on each equation of system (5.2) and using local energy estimates to eliminate the observation of the variable $q_{2}$. The result is the following.

Theorem 5.1. Assume that for some nonempty open set $\omega_{0} \subset \omega$, the coefficient $a_{21}$ verifies

$$
a_{21}^{n} \geq a_{0}>0 \quad \text { or } \quad-a_{21}^{n} \geq a_{0}>0 \quad \forall x \in \omega_{0}, n \in \llbracket 1, M \rrbracket .
$$

Then, there exist positive constants $C_{0}, C_{1}$ and $C_{2}$ such that for all $0<T<1$, under the condition $\triangle t \leq \min \left\{\widetilde{\triangle t},(8 \mathcal{M})^{-1}\right\}$ with

$$
\widetilde{\triangle t}=C_{0}\left(T+T^{2}+T^{2} \mathcal{M}^{2 / 3}\right)^{-5} T^{8}
$$


any solution to (5.2) satisfies

$$
\left|q_{1}^{\frac{1}{2}}\right|_{L^{2}(\Omega)}^{2}+\left|q_{2}^{\frac{1}{2}}\right|_{L^{2}(\Omega)}^{2} \leq C_{o b s}^{2}\left(f \int_{\omega \times(0, T)}\left|q_{1}\right|^{2}+e^{-\frac{C_{2}}{(\Delta t)^{1 / 5}}}\left[\left|\nabla q_{1}^{M+\frac{1}{2}}\right|_{L^{2}(\Omega)}^{2}+\left|\nabla q_{2}^{M+\frac{1}{2}}\right|_{L^{2}(\Omega)}^{2}\right]\right)
$$

with $C_{o b s}=e^{C_{1}\left(1+\frac{1}{T}+T \mathcal{M}^{2 / 3}+T \mathcal{M}\right)}$ and $\mathcal{M}=\max _{1 \leq i, j \leq 2}\left\|a_{i j}\right\|_{\infty}$.

This is an analogous result to the one presented in [13] for the observability of $m$-coupled equations with one control force. Indeed, we will adapt their proof and take into account the differences introduced by the time discretization scheme. It is worth noting that we can also extend our theorem for the case of coupled systems in cascade form (see condition (6) in [13]) but for convenience we only present a simpler case. Finally, we shall mention that for brevity we only present the proof of the observability inequality of (5.2), the controllability result for (5.1) can be readily obtained by arguing as in the previous sections.

Proof of Theorem 5.1. For the sake of clarity, we have divided the proof in three steps. We will keep track of the dependences of the constants. As before, since we are interested in controllability in small time, we have $\min \left\{T^{3}, T^{6}\right\}=T^{6}$. We also recall that system (5.2) can be rewritten in the more convenient form

$$
\begin{cases}-\left(\bar{D}_{t} q_{1}\right)^{n}-\Delta\left(\overline{\mathrm{t}}^{-} q_{1}\right)^{n}+a_{11}^{n}\left(\overline{\mathrm{t}}^{-} q_{1}\right)^{n}+a_{21}^{n}\left(\overline{\mathrm{t}}^{-} q_{2}\right)^{n}=0, & n \in \llbracket 1, M \rrbracket, \\ -\left(\bar{D}_{t} q_{2}\right)^{n}-\Delta\left(\overline{\mathrm{t}}^{-} q_{2}\right)^{n}+a_{12}^{n}\left(\overline{\mathrm{t}}^{-} q_{1}\right)^{n}+a_{22}^{n}\left(\overline{\mathrm{t}}^{-} q_{2}\right)^{n}=0, & n \in \llbracket 1, M \rrbracket, \\ q_{1}^{M+\frac{1}{2}}=q_{1, T}, \quad q^{M+\frac{1}{2}}=q_{2, T} & \end{cases}
$$

Step 1. Given $\omega_{0} \subset \omega$, we choose $\tilde{\omega} \subset \subset \omega_{0}$. We begin by applying the Carleman estimate (1.15) to each equation of (5.4). To abridge the notation, we have denoted by $\mathcal{I}(q)$ the left-hand side of $(1.15)$, thus we have

$$
\begin{aligned}
\mathcal{I}\left(q_{1}\right)+\mathcal{I}\left(q_{2}\right) \leq & C\left(\sum_{i=1}^{2} \iint_{\tilde{\omega} \times(0, T)} \overline{\mathrm{t}}^{-}\left(e^{2 s \varphi} s^{3}\right)\left(\overline{\mathrm{t}}^{-} q_{i}\right)^{2}+\sum_{i=1}^{2} \sum_{j=1}^{2}\left\|a_{j i}\right\|_{\infty}^{2} \iint_{Q}\left(\overline{\mathrm{t}}^{-} e^{2 s \varphi}\right)\left(\overline{\mathrm{t}}^{-} q_{j}\right)^{2}\right) \\
& +C(\triangle t)^{-1} \sum_{i=1}^{2}\left(\left|\left(e^{s \varphi} q_{i}\right)^{\frac{1}{2}}\right|_{L^{2}(\Omega)}^{2}+\left|\left(e^{s \varphi} q_{i}\right)^{M+\frac{1}{2}}\right|_{L^{2}(\Omega)}^{2}+\left|\left(e^{s \varphi} \nabla q_{i}\right)^{M+\frac{1}{2}}\right|_{L^{2}(\Omega)}^{2}\right)
\end{aligned}
$$

for all $\tau \geq \tau_{0}\left(T+T^{2}\right)$ and $\tau^{4} \triangle t\left(\delta^{4} T^{6}\right)^{-1} \leq \varepsilon_{0}$. As in the scalar case, all the lower order terms can be absorbed by taking $\tau$ large enough, this is

$$
\begin{aligned}
\mathcal{I}\left(q_{1}\right)+ & \mathcal{I}\left(q_{2}\right) \leq C\left(\iint_{\tilde{\omega} \times(0, T)} \overline{\mathrm{t}}^{-}\left(e^{2 s \varphi} s^{3}\right)\left(\overline{\mathrm{t}}^{-} q_{1}\right)^{2}+\iint_{\tilde{\omega} \times(0, T)} \overline{\mathrm{t}}^{-}\left(e^{2 s \varphi} s^{3}\right)\left(\overline{\mathrm{t}}^{-} q_{2}\right)^{2}\right) \\
& +C(\triangle t)^{-1} \sum_{i=1}^{2}\left(\left|\left(e^{s \varphi} q_{i}\right)^{\frac{1}{2}}\right|_{L^{2}(\Omega)}^{2}+\left|\left(e^{s \varphi} q_{i}\right)^{M+\frac{1}{2}}\right|_{L^{2}(\Omega)}^{2}+\left|\left(e^{s \varphi} \nabla q_{i}\right)^{M+\frac{1}{2}}\right|_{L^{2}(\Omega)}^{2}\right)
\end{aligned}
$$

for all

$$
\tau \geq \tau_{1}\left(T+T^{2}+T^{2} \max _{1 \leq i, j \leq 2}\left\|a_{j i}\right\|_{\infty}^{2 / 3}\right) .
$$

Step 2. Now, we will see that thanks to hypothesis (5.3), we can eliminate from (5.5) the observation term corresponding to $q_{2}$. To this end, consider two open sets $\tilde{\mathcal{O}}$ and $\mathcal{O}_{0}$ such that $\tilde{\omega} \subset \tilde{\mathcal{O}} \subset \subset \mathcal{O}_{0} \subset \omega_{0}$ and a cut-off function $\zeta \in C_{0}^{\infty}(\Omega)$ verifying

$$
\begin{gathered}
0 \leq \zeta \leq 1 \text { in } \Omega, \quad \zeta \equiv 1 \text { in } \tilde{\mathcal{O}}, \quad \operatorname{supp} \zeta \subset \mathcal{O}_{0} \\
\frac{\Delta \zeta}{\zeta^{1 / 2}} \in L^{\infty}(\Omega), \quad \frac{\nabla \zeta}{\zeta^{1 / 2}} \in L^{\infty}(\Omega)^{d}
\end{gathered}
$$

Such function exists. For obtaining condition (5.8) it suffices to take $\tilde{\zeta} \in C_{0}^{\infty}(\Omega)$ satisfying (5.7) and define $\zeta=\tilde{\zeta}^{4}$. In this way, $\zeta$ will verify both (5.7)-(5.8). 
By assumption, the coefficient $a_{21}$ satisfies (5.3) and, for convenience, we suppose that $a_{21}^{n} \geq a_{0}$ for all $x \in \Omega$ and $n \in \llbracket 1, M \rrbracket$. From (5.4), we multiply the equation satisfied by $q_{1}$ by $\overline{\mathrm{t}}^{-}\left(e^{2 s \varphi} s^{3}\right)^{n}\left(\overline{\mathrm{t}}^{-} q_{2}\right)^{n} \zeta$ in $L^{2}(\Omega)$ and sum over $n$. We get

$$
\begin{aligned}
a_{0} \iint_{\tilde{\mathcal{O}} \times(0, T)} \overline{\mathrm{t}}^{-}\left(e^{2 s \varphi} s^{3}\right)\left(\overline{\mathrm{t}}^{-} q_{2}\right)^{2} & \leq \iint_{Q} a_{21} \overline{\mathrm{t}}^{-}\left(e^{2 s \varphi} s^{3}\right)\left(\overline{\mathrm{t}}^{-} q_{2}\right)^{2} \zeta \\
& =\iint_{Q} \overline{\mathrm{t}}^{-}\left(e^{2 s \varphi} s^{3}\right)\left(\overline{\mathrm{t}}^{-} q_{2}\right)\left[\left(\bar{D}_{t} q_{1}\right)+\Delta\left(\overline{\mathrm{t}}^{-} q_{1}\right)-a_{11}\left(\overline{\mathrm{t}}^{-} q_{1}\right)\right] \zeta \\
& =: \sum_{i=1}^{3} K_{i} .
\end{aligned}
$$

We proceed to estimate each $K_{i}, 1 \leq i \leq 3$. Integrating by parts using formula (B.7) in the first term we obtain

$$
\begin{aligned}
K_{1}= & \iint_{Q}\left(\bar{D}_{t} q_{1}\right)\left(\overline{\mathrm{t}}^{-} e^{2 s \varphi} s^{3} q_{2}\right) \zeta \\
= & -\left(\left(e^{s \varphi} s^{3 / 2} q_{1} \zeta^{1 / 2}\right)^{\frac{1}{2}},\left(e^{s \varphi} s^{3 / 2} q_{2} \zeta^{1 / 2}\right)^{\frac{1}{2}}\right)_{L^{2}(\Omega)}+\left(\left(e^{s \varphi} s^{3 / 2} q_{1} \zeta^{1 / 2}\right)^{M+\frac{1}{2}},\left(e^{s \varphi} s^{3 / 2} q_{2} \zeta^{1 / 2}\right)^{M+\frac{1}{2}}\right)_{L^{2}(\Omega)} \\
& -\iint_{Q}\left(\overline{\mathrm{t}}^{+} q_{1}\right) \bar{D}_{t}\left(e^{2 s \varphi} s^{3} q_{2}\right) \zeta \\
= & : \sum_{j=1}^{3} K_{1}^{(j)}
\end{aligned}
$$

The first two terms can be easily bounded by using Hölder and Young inequalities, i.e.,

$$
\left|K_{1}^{(1)}\right|+\left|K_{1}^{(2)}\right| \leq C \sum_{i=1}^{2}\left(\left|\left(e^{s \varphi} s^{3 / 2} q_{i}\right)^{\frac{1}{2}}\right|_{L^{2}(\Omega)}^{2}+\left|\left(e^{s \varphi} s^{3 / 2} q_{i}\right)^{M+\frac{1}{2}}\right|_{L^{2}(\Omega)}^{2}\right) .
$$

By using formula (B.1) in the last term of (5.10), we obtain

$$
\begin{aligned}
K_{1}^{(3)} & =-\iint_{Q}\left(\overline{\mathrm{t}}^{+} q_{1}\right) \bar{D}_{t}\left(e^{2 s \varphi} s^{3} q_{2}\right) \zeta \\
& =-\iint_{Q}\left(\overline{\mathrm{t}}^{+} q_{1}\right) \overline{\mathrm{t}}^{-}\left(e^{2 s \varphi} s^{3}\right)\left(\bar{D}_{t} q_{2}\right) \zeta-\iint_{Q}\left(\overline{\mathrm{t}}^{+} q_{1}\right)\left(\overline{\mathrm{t}}^{+} q_{2}\right) \bar{D}_{t}\left(e^{2 s \varphi} s^{3}\right) \zeta \\
& =: H_{1}+H_{2} .
\end{aligned}
$$

From the fact that $\left(\overline{\mathrm{t}}^{+} q_{1}\right)=\triangle t \bar{D}_{t} q_{1}+\left(\overline{\mathrm{t}}^{-} q_{1}\right)$ and properties (5.7), we can use Hölder and Young inequalities to obtain

$\left|H_{1}\right| \leq \frac{\triangle t}{2} \sum_{i=1}^{2} \iint_{Q} \overline{\mathrm{t}}^{-}\left(e^{2 s \varphi} s^{3}\right)\left(\bar{D}_{t} q_{i}\right)^{2} \zeta+\gamma_{1} \iint_{Q} \overline{\mathrm{t}}^{-}\left(e^{2 s \varphi} s^{-1}\right)\left(\bar{D}_{t} q_{2}\right)^{2}+\frac{C}{\gamma_{1}} \iint_{\mathcal{O}_{0} \times(0, T)} \overline{\mathrm{t}}^{-}\left(e^{2 s \varphi} s^{7}\right)\left(\overline{\mathrm{t}}^{-} q_{1}\right)^{2}$

for any $\gamma_{1}>0$. Now, let us choose $\varepsilon_{1}$ small enough such that $\varepsilon_{1} \leq \min \left\{\varepsilon_{0}, a_{0} / 2 C\right\}$ where $C$ is the constant appearing in (5.5) and verifying

$$
\frac{\triangle t \tau^{4}}{\delta^{4} T^{8}} \leq \varepsilon_{1}
$$

Then, we have

$\left|H_{1}\right| \leq \varepsilon_{1} \sum_{i=1}^{2} \iint_{Q} \overline{\mathrm{t}}^{-}\left(e^{2 s \varphi} s^{-1}\right)\left(\bar{D}_{t} q_{i}\right)^{2}+\gamma_{1} \iint_{Q} \overline{\mathrm{t}}^{-}\left(e^{2 s \varphi} s^{-1}\right)\left(\bar{D}_{t} q_{2}\right)^{2}+\frac{C}{\gamma_{1}} \iint_{\mathcal{O}_{0} \times(0, T)} \overline{\mathrm{t}}^{-}\left(e^{2 s \varphi} s^{7}\right)\left(\overline{\mathrm{t}}^{-} q_{1}\right)^{2}$

Estimating the term $\mathrm{H}_{2}$ follows after a straightforward but long calculation since it involves the time derivative of the Carleman weight. Using formula (B.1), we have

$$
\bar{D}_{t}\left(e^{2 s \varphi} \theta^{3}\right)=\left(\overline{\mathrm{t}}^{+} e^{2 s \varphi}\right)\left(\bar{D}_{t} \theta^{3}\right)+\left(\overline{\mathrm{t}}^{-} \theta^{3}\right)\left(\bar{D}_{t} e^{2 s \varphi}\right) .
$$


Therefore,

$$
\begin{aligned}
H_{2} & =-\iint_{Q}\left(\overline{\mathrm{t}}^{+} q_{1}\right)\left(\overline{\mathrm{t}}^{+} q_{2}\right)\left(\overline{\mathrm{t}}^{+} e^{2 s \varphi}\right) \tau^{3}\left(\bar{D}_{t} \theta^{3}\right) \zeta-\iint_{Q}\left(\overline{\mathrm{t}}^{+} q_{1}\right)\left(\overline{\mathrm{t}}^{+} q_{2}\right)\left(\overline{\mathrm{t}}^{-} s^{3}\right)\left(\bar{D}_{t} e^{2 s \varphi}\right) \zeta \\
& =: I_{1}+I_{2} .
\end{aligned}
$$

Using formula (B.12) with $n=3$ on $I_{1}$ yields

$$
\left|I_{1}\right| \leq \iint_{Q}\left|\left(\overline{\mathrm{t}}^{+} q_{1}\right)\right|\left|\left(\overline{\mathrm{t}}^{+} q_{2}\right)\right| \overline{\mathrm{t}}^{+}\left(e^{2 s \varphi} s^{4}\right) \zeta+\iint_{Q}\left|\left(\overline{\mathrm{t}}^{+} q_{1}\right)\right|\left|\left(\overline{\mathrm{t}}^{+} q_{2}\right)\right|\left(\overline{\mathrm{t}}^{+} e^{2 s \varphi}\right) \frac{\triangle t \tau^{3}}{T^{8} \delta^{5}} \zeta
$$

Provided

$$
\frac{\triangle t \tau^{3}}{T^{8} \delta^{5}} \leq \varepsilon_{2}
$$

and using the properties of the function $\zeta$, we get after applying Hölder and Young inequalities that

$$
\begin{aligned}
\left|I_{1}\right| \leq & \gamma_{1} \iint_{Q}\left(\overline{\mathrm{t}}^{+} q_{2}\right)^{2}\left(\overline{\mathrm{t}}^{+} e^{2 s \varphi} s^{3}\right)+\frac{C}{\gamma_{1}} \iint_{\mathcal{O}_{0} \times(0, T)}\left(\overline{\mathrm{t}}^{+} q_{1}\right)^{2}\left(\overline{\mathrm{t}}^{+} e^{2 s \varphi} s^{5}\right) \\
& +\gamma_{1} \iint_{Q}\left(\overline{\mathrm{t}}^{+} q_{2}\right)^{2}\left(\overline{\mathrm{t}}^{+} e^{2 s \varphi}\right)+\frac{C}{\gamma_{1}} \iint_{\mathcal{O}_{0} \times(0, T)}\left(\overline{\mathrm{t}}^{+} q_{2}\right)^{2}\left(\overline{\mathrm{t}}^{+} e^{2 s \varphi}\right)
\end{aligned}
$$

for some positive constant $C$ only depending on $\varepsilon_{2}$. Modifying $\tau_{1}$, if necessary, so $(\tau \theta(t))^{-1} \leq 1$ for $t \in[0, T]$ and shifting the indices in the above expression, we get

$$
\begin{aligned}
\left|I_{1}\right| \leq & 2 \gamma_{1} \iint_{Q}\left(\overline{\mathrm{t}}^{-} q_{2}\right)^{2} \overline{\mathrm{t}}^{-}\left(e^{2 s \varphi} s^{3}\right)+\frac{C}{\gamma_{1}} \iint_{\mathcal{O}_{0} \times(0, T)}\left(\overline{\mathrm{t}}^{-} q_{1}\right)^{2} \overline{\mathrm{t}}^{-}\left(e^{2 s \varphi} s^{5}\right) \\
& +C \triangle t\left(\left|\left(e^{s \varphi} s^{5 / 2} q_{1}\right)^{M+\frac{1}{2}}\right|_{L^{2}(\Omega)}^{2}+\left|\left(e^{s \varphi} s^{3 / 2} q_{2}\right)^{M+\frac{1}{2}}\right|_{L^{2}(\Omega)}^{2}\right) .
\end{aligned}
$$

From Lemma B.4 and (B.13), we get

$$
\left|\left(\bar{D}_{t} e^{2 s \varphi}\right)\right| \leq C\left(\left(\overline{\mathrm{t}}^{+} e^{2 s \varphi}\right) \tau^{2}\left(\overline{\mathrm{t}}^{+} \theta\right)^{2}+\left(\overline{\mathrm{t}}^{+} e^{2 s \varphi}\right) \frac{\triangle t \tau^{2}}{\delta^{4} T^{6}}\right) .
$$

Arguing as above and provided (5.14) holds, we have

$$
\begin{aligned}
\left|I_{2}\right| \leq & 2 \gamma_{1} \iint_{Q}\left(\overline{\mathrm{t}}^{-} q_{2}\right)^{2} \overline{\mathrm{t}}^{-}\left(e^{2 s \varphi} s^{3}\right)+\frac{C}{\gamma_{1}} \iint_{\mathcal{O}_{0} \times(0, T)}\left(\overline{\mathrm{t}}^{-} q_{1}\right)^{2} \overline{\mathrm{t}}^{-}\left(e^{2 s \varphi} s^{7}\right) \\
& +C \triangle t\left(\left|\left(e^{s \varphi} s^{7 / 2} q_{1}\right)^{M+\frac{1}{2}}\right|_{L^{2}(\Omega)}^{2}+\left|\left(e^{s \varphi} s^{3 / 2} q_{2}\right)^{M+\frac{1}{2}}\right|_{L^{2}(\Omega)}^{2}\right) .
\end{aligned}
$$

Combining estimates (5.11) and (5.15)-(5.16), we can bound the first term of (5.9) as

$$
\begin{aligned}
\left|K_{1}\right| \leq & \varepsilon_{1} \sum_{i=1}^{2} \iint_{Q} \overline{\mathrm{t}}^{-}\left(e^{2 s \varphi} s^{-1}\right)\left(\bar{D}_{t} q_{i}\right)^{2}+5 \gamma_{1} \iint_{Q} \overline{\mathrm{t}}^{-}\left(e^{2 s \varphi} s^{3}\right)\left(\overline{\mathrm{t}}^{-} q_{2}\right)^{2}+\frac{C}{\gamma_{1}} \iint_{\mathcal{O}_{0} \times(0, T)} \overline{\mathrm{t}}^{-}\left(e^{2 s \varphi} s^{7}\right)\left(\overline{\mathrm{t}}^{-} q_{1}\right)^{2} \\
& +C \sum_{i=1}^{2}\left(\left|\left(e^{s \varphi} s^{3 / 2} q_{i}\right)^{\frac{1}{2}}\right|_{L^{2}(\Omega)}^{2}+\left|\left(e^{s \varphi} s^{3 / 2} q_{i}\right)^{M+\frac{1}{2}}\right|_{L^{2}(\Omega)}^{2}\right) \\
& +C \triangle t\left(\left|\left(e^{s \varphi} s^{7 / 2} q_{1}\right)^{M+\frac{1}{2}}\right|_{L^{2}(\Omega)}^{2}+\left|\left(e^{s \varphi} s^{3 / 2} q_{2}\right)^{M+\frac{1}{2}}\right|_{L^{2}(\Omega)}^{2}\right)
\end{aligned}
$$

For the term $K_{2}$ in $(5.9)$, we can integrate by parts in the space variable, thus

$$
\begin{aligned}
K_{2} & =\iint_{Q} \overline{\mathrm{t}}^{-}\left(e^{2 s \varphi} s^{3}\right) \zeta\left(\overline{\mathrm{t}}^{-} q_{2}\right) \Delta\left(\overline{\mathrm{t}}^{-} q_{1}\right) \\
& =-\iint_{Q} \nabla\left[\overline{\mathrm{t}}^{-}\left(e^{2 s \varphi} s^{3}\right) \zeta\right] \cdot \nabla\left(\overline{\mathrm{t}}^{-} q_{1}\right)\left(\overline{\mathrm{t}}^{-} q_{2}\right)-\iint_{Q} \overline{\mathrm{t}}^{-}\left(e^{2 s \varphi} s^{3}\right) \zeta \nabla\left(\overline{\mathrm{t}}^{-} q_{1}\right) \cdot \nabla\left(\overline{\mathrm{t}}^{-} q_{2}\right) .
\end{aligned}
$$


Using (5.8) is not difficult to show that

$$
\left|\nabla\left[\overline{\mathrm{t}}^{-}\left(e^{2 s \varphi} s^{3}\right) \zeta\right]\right| \leq C \overline{\mathrm{t}}^{-}\left(e^{2 s \varphi} s^{4}\right) \zeta^{1 / 2}
$$

and therefore, using Hölder and Young inequalities, we get

$$
\begin{aligned}
\left|K_{2}\right| \leq & \gamma_{1} \iint_{Q} \overline{\mathrm{t}}^{-}\left(e^{2 s \varphi} s^{3}\right)\left(\overline{\mathrm{t}}^{-} q_{2}\right)^{2}+\gamma_{2} \iint_{Q} \overline{\mathrm{t}}^{-}\left(e^{2 s \varphi} s\right)\left|\nabla\left(\overline{\mathrm{t}}^{-} q_{2}\right)\right|^{2} \\
& +C\left(\frac{1}{\gamma_{1}}+\frac{1}{\gamma_{2}}\right) \iint_{\mathcal{O}_{0} \times(0, T)} \overline{\mathrm{t}}^{-}\left(e^{2 s \varphi} s^{5}\right)\left|\nabla\left(\overline{\mathrm{t}}^{-} q_{1}\right)\right|^{2}
\end{aligned}
$$

for any $\gamma_{2}>0$.

For $K_{3}$, we readily have

$$
\left|K_{3}\right| \leq \gamma_{1} \iint_{Q} \overline{\mathrm{t}}^{-}\left(e^{2 s \varphi} s^{3}\right)\left(\overline{\mathrm{t}}^{-} q_{2}\right)^{2}+\frac{C}{\gamma_{1}} \iint_{\mathcal{O}_{0} \times(0, T)} \overline{\mathrm{t}}^{-}\left(e^{2 s \varphi} s^{3}\right)\left(\overline{\mathrm{t}}^{-} q_{1}\right)^{2} .
$$

Recalling that we have chosen $\tau_{1}$ large enough so that $(\tau \theta(t))^{-1} \leq 1$, we can combine (5.17)-(5.19) to obtain the local energy estimate

$$
\begin{aligned}
a_{0} \iint_{\tilde{\mathcal{O}} \times(0, T)} \overline{\mathrm{t}}^{-}\left(e^{2 s \varphi} s^{3}\right)\left(\overline{\mathrm{t}}^{-} q_{2}\right)^{2} \\
\leq \varepsilon_{1} \sum_{i=1}^{2} \iint_{Q} \overline{\mathrm{t}}^{-}\left(e^{2 s \varphi} s^{-1}\right)\left(\bar{D}_{t} q_{i}\right)^{2}+7 \gamma_{1} \iint_{Q} \overline{\mathrm{t}}^{-}\left(e^{2 s \varphi} s^{3}\right)\left(\overline{\mathrm{t}}^{-} q_{2}\right)^{2} \\
\quad+\gamma_{2} \iint_{Q} \overline{\mathrm{t}}^{-}\left(e^{2 s \varphi} s\right)\left|\nabla\left(\overline{\mathrm{t}}^{-} q_{2}\right)\right|^{2}+\frac{C}{\gamma_{1}} \iint_{\mathcal{O}_{0} \times(0, T)} \overline{\mathrm{t}}^{-}\left(e^{2 s \varphi} s^{7}\right)\left(\overline{\mathrm{t}}^{-} q_{1}\right)^{2} \\
\quad+C\left(\frac{1}{\gamma_{1}}+\frac{1}{\gamma_{2}}\right) \iint_{\mathcal{O}_{0} \times(0, T)} \overline{\mathrm{t}}^{-}\left(e^{2 s \varphi} s^{5}\right)\left|\nabla\left(\overline{\mathrm{t}}^{-} q_{1}\right)\right|^{2} \\
\quad+C(\triangle t)^{-1} \sum_{i=1}^{2}\left(\left|\left(e^{s \varphi} q_{i}\right)^{\frac{1}{2}}\right|_{L^{2}(\Omega)}^{2}+\left|\left(e^{s \varphi} q_{i}\right)^{M+\frac{1}{2}}\right|_{L^{2}(\Omega)}^{2}\right)
\end{aligned}
$$

where we have used condition (5.14) to simplify the last term. Replacing (5.20) in (5.5), and taking $\gamma_{i}$ small enough, we obtain

$$
\begin{aligned}
\mathcal{I}\left(q_{1}\right)+ & \mathcal{I}\left(q_{2}\right) \leq C\left(\iint_{\mathcal{O}_{0} \times(0, T)} \overline{\mathrm{t}}^{-}\left(e^{2 s \varphi} s^{7}\right)\left(\overline{\mathrm{t}}^{-} q_{1}\right)^{2}+\iint_{\mathcal{O}_{0} \times(0, T)} \overline{\mathrm{t}}^{-}\left(e^{2 s \varphi} s^{5}\right)\left|\nabla\left(\overline{\mathrm{t}}^{-} q_{1}\right)\right|^{2}\right) \\
& +C(\triangle t)^{-1} \sum_{i=1}^{2}\left(\left|\left(e^{s \varphi} q_{i}\right)^{\frac{1}{2}}\right|_{L^{2}(\Omega)}^{2}+\left|\left(e^{s \varphi} q_{i}\right)^{M+\frac{1}{2}}\right|_{L^{2}(\Omega)}^{2}+\left|\left(e^{s \varphi} \nabla q_{i}\right)^{M+\frac{1}{2}}\right|_{L^{2}(\Omega)}^{2}\right) .
\end{aligned}
$$

Step 3. To eliminate the local term corresponding to $\nabla\left(\overline{\mathrm{t}}^{-} q_{1}\right)$, we consider a function $\eta \in C_{0}^{\infty}(\Omega)$ with properties analogous to (5.7)-(5.8) chosen for the sets $\mathcal{O}_{0}$ and $\omega_{0}$. In this way, we have

$$
\begin{aligned}
\iint_{\mathcal{O}_{0} \times(0, T)} \overline{\mathrm{t}}^{-}\left(e^{2 s \varphi} s^{5}\right)\left|\nabla\left(\overline{\mathrm{t}}^{-} q_{1}\right)\right|^{2} \leq & \iint_{Q} \overline{\mathrm{t}}^{-}\left(e^{2 s \varphi} s^{5}\right)\left|\nabla\left(\overline{\mathrm{t}}^{-} q_{1}\right)\right|^{2} \eta \\
= & -\iint_{Q} \overline{\mathrm{t}}^{-}\left(e^{2 s \varphi} s^{5}\right) \Delta\left(\overline{\mathrm{t}}^{-} q_{1}\right)\left(\overline{\mathrm{t}}^{-} q_{1}\right) \eta \\
& -\iint_{Q} \nabla\left[\overline{\mathrm{t}}^{-}\left(e^{2 s \varphi} s^{5}\right) \eta\right] \cdot \nabla\left(\overline{\mathrm{t}}^{-} q_{1}\right)\left(\overline{\mathrm{t}}^{-} q_{1}\right),
\end{aligned}
$$

where we have integrated by parts. Using that $\left|\nabla\left[\overline{\mathrm{t}}^{-}\left(e^{2 s \varphi} s^{5}\right) \eta\right]\right| \leq C \overline{\mathrm{t}}^{-}\left(e^{2 s \varphi} s^{6}\right) \eta^{1 / 2}$, we readily have

$$
\begin{aligned}
\iint_{\mathcal{O}_{0} \times(0, T)} \overline{\mathrm{t}}^{-}\left(e^{2 s \varphi} s^{5}\right)\left|\nabla\left(\overline{\mathrm{t}}^{-} q_{1}\right)\right|^{2} \leq & \gamma_{3} \iint_{Q} \overline{\mathrm{t}}^{-}\left(e^{2 s \varphi} s^{-1}\right)\left|\Delta\left(\overline{\mathrm{t}}^{-} q_{1}\right)\right|^{2}+\gamma_{4} \iint_{Q} \overline{\mathrm{t}}^{-}\left(e^{s \varphi} s\right)\left|\nabla\left(\overline{\mathrm{t}}^{-} q_{1}\right)\right|^{2} \\
& +C\left(\frac{1}{\gamma_{3}}+\frac{1}{\gamma_{4}}\right) \iint_{\omega_{0} \times(0, T)} \overline{\mathrm{t}}^{-}\left(e^{2 s \varphi} s^{11}\right)\left(\overline{\mathrm{t}}^{-} q_{1}\right)^{2}
\end{aligned}
$$


for any $\gamma_{3}, \gamma_{4}>0$. Using estimate (5.22) in (5.21) and taking the parameters $\gamma_{i}$ small enough, we obtain

$$
\begin{aligned}
\mathcal{I}\left(q_{1}\right)+ & \mathcal{I}\left(q_{2}\right) \leq C\left(\iint_{\omega_{0} \times(0, T)} \overline{\mathrm{t}}^{-}\left(e^{2 s \varphi} s^{11}\right)\left(\overline{\mathrm{t}}^{-} q_{1}\right)^{2}\right) \\
& +C(\triangle t)^{-1} \sum_{i=1}^{2}\left(\left|\left(e^{s \varphi} q_{i}\right)^{\frac{1}{2}}\right|_{L^{2}(\Omega)}^{2}+\left|\left(e^{s \varphi} q_{i}\right)^{M+\frac{1}{2}}\right|_{L^{2}(\Omega)}^{2}+\left|\left(e^{s \varphi} \nabla q_{i}\right)^{M+\frac{1}{2}}\right|_{L^{2}(\Omega)}^{2}\right)
\end{aligned}
$$

for all $\tau$ as in (5.6) and $\triangle t \tau^{3}\left(T^{8} \delta^{5}\right)^{-1} \leq \varepsilon_{2}$, with $C>0$ only depending on $\Omega, \omega$ and $a_{0}$.

Step 4. Following the proof of Proposition 3.1, for $\triangle t \max _{1 \leq i, j \leq 2}\left\|a_{i j}\right\|_{\infty}<1 / 8$, we can obtain the estimate

$$
\sum_{i=1}^{2}\left|q_{i}^{\frac{1}{2}}\right|_{L^{2}(\Omega)}^{2} \leq e^{C T \max _{1 \leq i, j \leq 2}\left\|a_{i j}\right\|_{\infty}} \sum_{i=1}^{2}\left|q_{i}^{n+\frac{1}{2}}\right|_{L^{2}(\Omega)}^{2}, \quad \forall n \in \llbracket 1, M \rrbracket .
$$

Moreover, from (3.8) and (5.24), we get

$$
\sum_{i=1}^{2} \iint_{Q} \overline{\mathrm{t}}^{-}\left(e^{2 s \varphi} s^{3}\right)\left(\overline{\mathrm{t}}^{-} q_{i}\right)^{2} \geq C T e^{-\frac{C \tau}{T^{2}}-C T \max _{1 \leq i, j \leq 2}\left\|a_{i j}\right\|_{\infty}} \sum_{i=1}^{2}\left|q_{i}^{\frac{1}{2}}\right|_{L^{2}(\Omega)}^{2} .
$$

Using Poincaré inequality and (5.24), we have that the last three terms of (5.23) can be bounded as follows

$$
\begin{aligned}
\sum_{i=1}^{2}\left(\left|\left(e^{s \varphi} q_{i}\right)^{\frac{1}{2}}\right|_{L^{2}(\Omega)}^{2}+\left|\left(e^{s \varphi} q_{i}\right)^{M+\frac{1}{2}}\right|_{L^{2}(\Omega)}^{2}\right. & \left.+\left|\left(e^{s \varphi} \nabla q_{i}\right)^{M+\frac{1}{2}}\right|_{L^{2}(\Omega)}^{2}\right) \\
\leq & e^{-C \frac{\tau}{\delta T^{2}}+C T \max _{1 \leq i, j \leq 2}\left\|a_{i j}\right\|_{\infty}} \sum_{i=1}^{2}\left|\nabla q_{i}^{M+\frac{1}{2}}\right|_{L^{2}(\Omega)}^{2} .
\end{aligned}
$$

On the other hand, observe that if $\tau \geq \frac{11}{8 k_{0} T^{2}}$, the weight function $e^{2 s \varphi} s^{11}$ is uniformly bounded for all $(x, t) \in Q$ (cf. (3.11)). Therefore, from estimates (5.23) and (5.25)-(5.26), we obtain

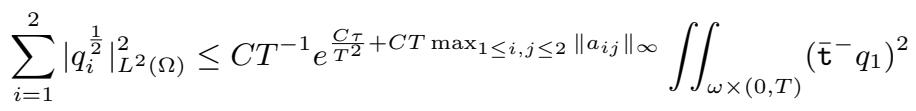

$$
\begin{aligned}
& +e^{-\frac{C \tau}{\delta T^{2}}+C T \max _{1 \leq i, j \leq 2}\left\|a_{i j}\right\|_{\infty}} \sum_{i=1}^{2}\left|\nabla q_{i}^{M+\frac{1}{2}}\right|_{L^{2}(\Omega)}^{2}
\end{aligned}
$$

for any $\tau \geq \tau_{2}\left(T+T^{2}+T^{2} \max _{1 \leq i . j \leq 2}\left\|a_{i j}\right\|_{\infty}\right)$ with $\tau_{2}=\max \left\{\tau_{1}, 11 / 8 k_{0}\right\}$ and any $\delta$ small enough. To conclude the proof, we have will fix the parameters involved. Recall that the condition

$$
\frac{\triangle t \tau^{5}}{T^{8} \delta^{5}} \leq \varepsilon_{2}
$$

has to be fullfilled along $0<\delta \leq \delta_{1}$. We fix $\tau=\tau_{2}\left(T+T^{2}+T^{2} \max _{1 \leq i, j \leq 2}\left\|a_{i j}\right\|_{\infty}\right)$ and define

$$
\widetilde{\triangle t}:=\frac{\varepsilon_{2}}{\tau_{2}^{5}} \delta_{1}^{5}\left(T+T^{2}+T^{2} \max _{1 \leq i, j \leq 2}\left\|a_{i j}\right\|_{\infty}^{2 / 3}\right)^{-5} T^{8}
$$

which gives

$$
\frac{\tau^{5} \widetilde{\triangle t}}{\delta_{1}^{5} T^{8}}=\varepsilon_{2}
$$

Now, we choose $\triangle t \leq \min \{\widetilde{\triangle t}, 1 / 8 \mathcal{M}\}$ and set $\delta=\frac{(\triangle t)^{1 / 5} \delta_{1}}{(\widetilde{\triangle t})^{1 / 5}} \leq \delta_{1}$. We find then

$$
\frac{\tau^{5} \triangle t}{\delta^{5} T^{8}}=\varepsilon_{2}
$$

Therefore, $\tau /\left(T^{2} \delta\right)=\left(\varepsilon_{2} T^{-2}\right)^{1 / 5} /(\triangle t)^{1 / 5}$ and from (5.27) we get

$$
\sum_{i=1}^{2}\left|q_{i}^{\frac{1}{2}}\right|_{L^{2}(\Omega)}^{2} \leq e^{C_{1}\left(1+\frac{1}{T}+\mathcal{M}^{2 / 3}+T \mathcal{M}\right)}\left(\iint_{\omega \times(0, T)}\left|q_{1}\right|^{2}+e^{-\frac{C_{2}}{(\Delta t)^{1 / 5} T^{2 / 5}}} \sum_{i=1}^{2}\left|\nabla q_{i}^{M+\frac{1}{2}}\right|_{L^{2}(\Omega)}^{2}\right) .
$$

This concludes the proof. 
Remark 5.2. The approach presented here can be used to address other well-known control problems for coupled systems in the discrete setting. To fix ideas, let $\mathcal{O} \subset \Omega$ be an observation subset and consider the functional

$$
\Psi(y)=\frac{1}{2} \sum_{n=1}^{M} \triangle t\left|y^{n}\right|_{L^{2}(\mathcal{O})}^{2}
$$

and the control system

$$
\begin{cases}\frac{y^{n+1}-y^{n}}{\triangle t}-\Delta y^{n+1}=\mathbf{1}_{\omega} v^{n+\frac{1}{2}}+\xi^{n+\frac{1}{2}}, & n \in \llbracket 0, M-1 \rrbracket, \\ y_{\mid \partial \Omega}^{n+1}=0, & n \in \llbracket 0, M-1 \rrbracket, \\ y^{0}=y_{0}+\sigma w_{0}, & \end{cases}
$$

where $y_{0} \in L^{2}(\Omega)$ and $\xi \in L_{\mathcal{D}}^{2}(Q)$ are given functions and the data of equation (5.28) is incomplete in the following sense: $w_{0} \in L^{2}(\Omega)$ is unknown with $\left|w_{0}\right|_{L^{2}(\Omega)}=1$ and $\sigma \in \mathbb{R}$ is unknown and small enough. The idea is to look for a control $v=\left(v^{n+\frac{1}{2}}\right)_{n \in \llbracket 0, M-1 \rrbracket}$ such that

$$
\left.\frac{\partial \Psi(y)}{\partial \sigma}\right|_{\sigma=0}=0, \quad \forall w_{0} \in L^{2}(\Omega)
$$

This is the so-called insensitizing problem (see the seminal work [17]) and has been thoroughly studied in different contexts, see, for instance, [1, 14, 18, 19, 20].

As classical in this framework, the insensitizing control problem is equivalent to study the nullcontrollability of a cascade system of parabolic PDEs (see, e.g., [19, Theorem 1]). At the discrete level, (5.29) translates into finding a control $v$ such that

$$
q^{\frac{1}{2}}=0
$$

where $q=\left(q^{n+\frac{1}{2}}\right)_{n \in \llbracket 0, M-1 \rrbracket}$ can be found from the following forward-backward cascade system

$$
\begin{aligned}
& \begin{cases}\frac{y^{n+1}-y^{n}}{\triangle t}-\Delta y^{n+1}=\mathbf{1}_{\omega} v^{n+\frac{1}{2}}+\xi^{n+\frac{1}{2}}, & n \in \llbracket 0, M-1 \rrbracket, \\
y_{\mid \partial \Omega}^{n+1}=0, & n \in \llbracket 0, M-1 \rrbracket, \\
y^{0}=y_{0}, & \end{cases} \\
& \begin{cases}\frac{q^{n-\frac{1}{2}}-q^{n+\frac{1}{2}}}{\triangle t}-\Delta q^{n-\frac{1}{2}}=\mathbf{1}_{\mathcal{O}} y^{n}, & n \in \llbracket 1, M \rrbracket, \\
q_{\mid \partial \Omega}^{n-\frac{1}{2}}=0, & n \in \llbracket 1, M \rrbracket, \\
q^{M+\frac{1}{2}}=0 . & \end{cases}
\end{aligned}
$$

However, as discussed in Section 1, we cannot expect to obtain such kind of result for (5.28) but rather to obtain a relaxed condition. In view of previous results for discrete-in-space insensitizing problems (see [3, Theorem 1.4]), the Carleman inequalities (1.15) and (1.16) and the procedure developed in this section, we can expect that a relaxed notion of $\phi(\triangle t)$-insensitizing control also holds for (5.28).

\section{A Proof of Lemma 2.3}

Proof of Lemma 2.3. We begin by increasing the value of $\tau_{1}$, if necessary, so that $\tau_{1} \geq 1$ and $\tau \geq 1$. Notice that

$$
1 \leq \tau_{1}(1 / T+1) \leq \tau \theta(t)=s(t), \quad \text { for all } 0 \leq t \leq T
$$

Now, we rewrite $\underline{X}=\sum_{i=1}^{5} X^{(i)}$. Provided

$$
\frac{\tau \triangle t}{T^{4} \delta^{3}} \leq \varepsilon_{1}
$$

holds with $\varepsilon_{1}>0$ small enough and from (A.1), we have

$$
X^{(1)} \leq \varepsilon_{1}\left(\iint_{Q}\left(\overline{\mathrm{t}}^{-} s\right)^{3}\left(\overline{\mathrm{t}}^{-} z\right)^{2}\right),
$$


In the same way, it is not difficult to see that

$$
X^{(2)} \leq \varepsilon_{2}\left(\iint_{Q}\left(\overline{\mathrm{t}}^{-} s\right)^{3}\left(\overline{\mathrm{t}}^{-} z\right)^{2}\right)
$$

for some $\varepsilon_{2}>0$ sufficiently small such that

$$
\frac{\tau^{2} \triangle t}{T^{6} \delta^{4}} \leq \varepsilon_{2}
$$

Moreover, we have

$$
X^{(3)} \leq \varepsilon_{3}\left(\iint_{Q}\left(\overline{\mathrm{t}}^{-} s\right)^{3}\left(\overline{\mathrm{t}}^{-} z\right)^{2}\right)
$$

for some $\varepsilon_{3}>0$ such that

$$
\frac{\tau^{2} \triangle t}{T^{4} \delta^{3}} \leq \varepsilon_{3}
$$

Using the expressions verified by (A.2) and (A.3), the last two terms of $\underline{X}$ can be bounded as

$$
X^{(4)}+X^{(5)} \leq \varepsilon_{1}^{2}\left(\iint_{Q}\left(\overline{\mathrm{t}}^{-} s\right)^{3}\left(\overline{\mathrm{t}}^{-} z\right)^{2}\right)+\varepsilon_{2}^{2}\left(\iint\left(\overline{\mathrm{t}}^{-} s\right)^{3}\left(\overline{\mathrm{t}}^{-} z\right)^{2}\right)
$$

Reasoning as above, we can also deduce bounds for the terms in $\underline{Y}$. Using a similar notation and from the fact that $\max _{t \in[0, T]} \theta \leq\left(\delta T^{2}\right)^{-1}$, we get

$$
\begin{aligned}
Y^{(1)} & =\Delta t \tau^{2} \iint_{Q}\left(\overline{\mathrm{t}}^{-} \theta\right)^{2}\left(\bar{D}_{t} z\right)^{2}=\Delta t \tau^{3} \iint_{Q}\left(\overline{\mathrm{t}}^{-} \theta\right)^{3}\left(\bar{D}_{t} z\right)^{2}\left(\overline{\mathrm{t}}^{-} s\right)^{-1} \\
& \leq \varepsilon_{4}\left(\iint_{Q}\left(\overline{\mathrm{t}}^{-} s\right)^{-1}\left(\bar{D}_{t} z\right)^{2}\right)
\end{aligned}
$$

where the condition

$$
\frac{\triangle t \tau^{3}}{T^{6} \delta^{3}} \leq \varepsilon_{4}
$$

holds for some $\varepsilon_{4}>0$ small enough.

In the same spirit, we get

$$
\begin{aligned}
Y^{(2)} & =T \tau \Delta t \iint_{Q}\left(\overline{\mathrm{t}}^{-} \theta\right)^{2}\left(\bar{D}_{t} z\right)^{2}=T \tau^{2} \Delta t \iint_{Q}\left(\overline{\mathrm{t}}^{-} \theta\right)^{3}\left(\bar{D}_{t} z\right)^{2}\left(\overline{\mathrm{t}}^{-} s\right)^{-1} \\
& \leq \varepsilon_{5} \iint_{Q}\left(\overline{\mathrm{t}}^{-} s\right)^{-1}\left(\bar{D}_{t} z\right)^{2}
\end{aligned}
$$

provided the following condition

$$
\frac{\tau^{2} \triangle t}{\delta^{3} T^{5}} \leq \varepsilon_{5}
$$

A straightforward computation yields

$$
Y^{(3)} \leq \varepsilon_{6}^{2}\left(\iint_{Q}\left(\overline{\mathrm{t}}^{-} s\right)^{-1}\left(\bar{D}_{t} z\right)^{2}\right) .
$$

for some $\varepsilon_{6}$ verifying

$$
\frac{\tau \triangle t}{\delta^{2} T^{3}} \leq \varepsilon_{6}
$$

For the fourth term of $\underline{Y}$, we may use (A.1) to write

$$
\begin{aligned}
Y^{(4)} & =\tau^{2}(\triangle t)^{2} T^{2} \iint_{Q}\left(\overline{\mathrm{t}}^{-} \theta\right)^{4}\left(\bar{D}_{t} z\right)^{2} \\
& \leq \tau^{4}(\triangle t)^{2} T^{2} \iint_{Q}\left(\overline{\mathrm{t}}^{-} \theta\right)^{6}\left(\bar{D}_{t} z\right)^{2}\left(\overline{\mathrm{t}}^{-} s\right)^{-1}
\end{aligned}
$$


Hence, if condition (A.6) holds, we have

$$
Y^{(4)} \leq \varepsilon_{5}^{2}\left(\iint_{Q}\left(\overline{\mathrm{t}}^{-} s\right)^{-1}\left(\bar{D}_{t} z\right)^{2}\right) .
$$

For the last term of $\underline{Y}$, using again (A.1) and arguing as above, we obtain

$$
Y^{(5)} \leq \varepsilon_{0}^{4}\left(\tau^{-1} \iint_{Q}\left(\bar{D}_{t} z\right)^{2}\left(\overline{\mathrm{t}}^{-} \theta\right)^{-1}\right) .
$$

whenever (2.31) holds.

Recall that $\delta \leq 1 / 2$ and $\tau \geq 1$, then conditions (A.2)-(A.7) can be simplified into a general one verifying

$$
\frac{\triangle t \tau^{4}}{\min \left\{T^{3}, T^{6}\right\} \delta^{4}} \leq \varepsilon
$$

for some $\varepsilon=\varepsilon\left(\lambda_{0}\right)$ small enough. Collecting the estimates for $X^{(i)}$ and $Y^{(i)}, i=1, \ldots, 5$, we obtain the desired inequality (2.34). This concludes the proof.

\section{B Some technical lemmas}

The goal of this appendix is to summarize a series of tools that allow to manipulate the discrete operators $D_{t}$ and $\bar{D}_{t}$ and provide estimates for the application of such operators on the weight functions.

To avoid introducing additional notation, we introduce the following continuous difference operator. For a function $f$ defined on $\mathbb{R}$, we set

$$
\begin{aligned}
& \mathrm{t}^{+} f(t):=f(t+\Delta t), \quad \mathrm{t}^{-} f(t):=f(t), \\
& \mathrm{D}_{t} f:=\frac{1}{\Delta t}\left(\mathrm{t}^{+}-\mathrm{t}^{-}\right) f .
\end{aligned}
$$

In this way, discrete versions of the results given below will be natural. With the notation given in the introduction, for a function $f$ continuosly defined on $\mathbb{R}$, the discrete function $D_{t} f$ is in fact $\mathrm{D}_{t} f$ evaluated at the mesh points $\mathcal{P}$ and $\bar{D}_{t} f$ amounts to evaluate $\mathrm{D}_{t} f$ at the points $\mathcal{D}$. This can be readily seen just by considering the change of variables $t \mapsto t-\frac{\Delta t}{2}$.

Lemma B.1. Let the functions $f_{1}$ and $f_{2}$ be continuously defined over $\mathbb{R}$. We have

$$
\mathrm{D}_{t}\left(f_{1} f_{2}\right)=\mathrm{t}^{+} f_{1} \mathrm{D}_{t} f_{2}+\mathrm{D}_{t} f_{1} \mathrm{t}^{-} f_{2} .
$$

The same holds for

$$
\mathrm{D}_{t}\left(f_{1} f_{2}\right)=\mathrm{t}^{-} f_{1} \mathrm{D}_{t} f_{2}+\mathrm{D}_{t} f_{1} \mathrm{t}^{+} f_{2} .
$$

From the above formulas, if $f_{1}=f_{2}=f$, we have the useful identities

$$
\mathrm{t}^{+} f \mathrm{D}_{t} f=\frac{1}{2} \mathrm{D}_{t}\left(f^{2}\right)+\frac{1}{2} \triangle t\left(\mathrm{D}_{t} f\right)^{2}, \quad \mathrm{t}^{-} f \mathrm{D}_{t} f=\frac{1}{2} \mathrm{D}_{t}\left(f^{2}\right)-\frac{1}{2} \Delta t\left(\mathrm{D}_{t} f\right)^{2} .
$$

The translation of the result to discrete functions $f, g_{1}, g_{2} \in H^{\overline{\mathcal{D}}}$ is

$$
\begin{aligned}
& \bar{D}_{t}\left(g_{1} g_{2}\right)=\left(\overline{\mathrm{t}}^{+} g_{1}\right) \bar{D}_{t} g_{2}+\bar{D}_{t} g_{1}\left(\overline{\mathrm{t}}^{-} g_{2}\right), \\
& \bar{D}_{t}\left(g_{1} g_{2}\right)=\left(\overline{\mathrm{t}}^{-} g_{1}\right) \bar{D}_{t} g_{2}+\bar{D}_{t} g_{1}\left(\overline{\mathrm{t}}^{+} g_{2}\right),
\end{aligned}
$$

and

$$
\begin{aligned}
& \left(\overline{\mathrm{t}}^{+} f\right) \bar{D}_{t} f=\frac{1}{2} \bar{D}_{t}\left(f^{2}\right)+\frac{1}{2} \triangle t\left(\bar{D}_{t} f\right)^{2}, \\
& \left(\overline{\mathrm{t}}^{-} f\right) \bar{D}_{t} f=\frac{1}{2} \bar{D}_{t}\left(f^{2}\right)-\frac{1}{2} \triangle t\left(\bar{D}_{t} f\right)^{2} .
\end{aligned}
$$

Of course, the above identities also hold for functions $f, g_{1}, g_{2} \in H^{\overline{\mathcal{P}}}$ and their respective translation operators and difference operator $\mathrm{t}^{ \pm}$and $D_{t}$.

The following result cover discrete integration by parts and some useful related formulas. 
Proposition B.2. Let $\left\{H,(\cdot, \cdot)_{H}\right\}$ be a real Hilbert space and consider $u \in H^{\overline{\mathcal{P}}}$ and $v \in H^{\overline{\mathcal{D}}}$. We have the following:

$$
\begin{aligned}
& \int_{0}^{T}\left(\mathrm{t}^{+} u, v\right)_{H}=\int_{0}^{T}\left(u, \overline{\mathrm{t}}^{-} v\right)_{H} \\
& f_{0}^{T}\left(\mathrm{t}^{-} u, v\right)_{H}=\Delta t\left(u^{0}, v^{\frac{1}{2}}\right)_{H}-\triangle t\left(u^{M}, v^{M+\frac{1}{2}}\right)_{H}+\int_{0}^{T}\left(u, \overline{\mathrm{t}}^{+} v\right)_{H} .
\end{aligned}
$$

Moreover, combining the above identities, we have the following discrete integration by parts formula

$$
f_{0}^{T}\left(D_{t} u, v\right)_{H}=-\left(u^{0}, v^{\frac{1}{2}}\right)_{H}+\left(u^{M}, v^{M+\frac{1}{2}}\right)_{H}-\int_{0}^{T}\left(\bar{D}_{t} v, u\right)_{H} .
$$

Remark B.3. If we consider two functions $f, g \in H^{\overline{\mathcal{D}}}$, we can combine (B.4) and (B.6) to obtain the formula

$$
\int_{0}^{T}\left(\bar{D}_{t} f, \overline{\mathrm{t}}^{-} g\right)_{H}=-\left(f^{\frac{1}{2}}, g^{\frac{1}{2}}\right)_{H}+\left(f^{M+\frac{1}{2}}, g^{M+\frac{1}{2}}\right)_{H}-\int_{0}^{T}\left(\overline{\mathrm{t}}^{+} f, \bar{D}_{t} g\right)_{H} .
$$

Analogously, for $f, g \in H^{\overline{\mathcal{P}}}$, the following holds

$$
f_{0}^{T}\left(D_{t} f, \mathrm{t}^{+} g\right)_{H}=-\left(f^{0}, g^{0}\right)_{H}+\left(f^{M}, g^{M}\right)_{H}-f_{0}^{T}\left(\mathrm{t}^{-} f, D_{t} g\right)_{H} .
$$

Observe that in this formulas, the integrals are taken over the same discrete points. These will be particularly useful during the derivation of the Carleman estimates (1.15) and (1.16).

We present here some technical results of discrete operations performed on the Carleman weights. These are of particular interest in the demonstration of Theorem 1.4 and for dealing with coupled parabolic problems as in Section 5. To be consistent with Section 1.4, we set $r=e^{s \varphi}$ and $\rho=r^{-1}$. We highlight the dependence on $\tau, \delta, \triangle t$ and $\lambda$ in the following estimates.

Lemma B.4 (Derivative of the Carleman weight). Provided $\triangle t \tau\left(T^{3} \delta^{2}\right)^{-1} \leq \kappa$, we have

$$
\mathrm{t}^{-}(\rho) \mathrm{D}_{t} r=\tau \mathrm{t}^{-}\left(\theta^{\prime}\right) \phi+\triangle t\left(\frac{\tau}{\delta^{3} T^{4}}+\frac{\tau^{2}}{\delta^{4} T^{6}}\right) \mathcal{O}_{\lambda, \kappa}(1) .
$$

Proof. The result follows from the Taylor formula

$$
f(t+y)=\sum_{j=0}^{n-1} \frac{y^{j}}{j !} f^{(j)}(t)+y^{n} \int_{0}^{1} \frac{(1-\sigma)^{n-1}}{(n-1) !} f^{(n)}(t+\sigma y) d \sigma
$$

at order $n=2$. Applying formula (B.10) with $f=e^{s \varphi}$ and $y=\Delta t$, we have

$$
\frac{e^{s(t+\Delta t) \varphi}-e^{s(t) \varphi}}{\triangle t}=\tau \theta^{\prime}(t) \varphi e^{s(t) \varphi}+\triangle t \int_{0}^{1}(1-\sigma) \partial_{t}^{2} e^{s(\cdot+\sigma \Delta t) \varphi} d \sigma .
$$

Taking the second derivative of the weight function and factorizing the term $e^{s(t) \varphi}$, we obtain

$$
\begin{aligned}
& \frac{e^{s(t+\Delta t) \varphi}-e^{s(t) \varphi}}{\Delta t}=\left(\tau \theta^{\prime}(t) \varphi+\triangle t \int_{0}^{1}(1-\sigma) e^{\tau \theta(t+\sigma \triangle t) \varphi-\tau \theta(t) \varphi} \tau \theta^{\prime \prime}(t+\sigma \triangle t) \varphi d \sigma\right. \\
& \left.+\triangle t \int_{0}^{1}(1-\sigma) e^{\tau \theta(t+\sigma \triangle t) \varphi-\tau \theta(t) \varphi} \tau^{2}\left(\theta^{\prime}\right)^{2}(t+\sigma \triangle t) \varphi^{2} d \sigma\right) e^{\tau \theta(t) \varphi}
\end{aligned}
$$

This expression, together with fact that $\|\varphi\|_{C(\bar{\Omega})}=\mathcal{O}_{\lambda}(1)$ and

$$
\max _{t \in[0, T]} \theta^{(j)} \leq \frac{1}{\delta^{j+1} T^{j+2}}, \quad j=0,1, \ldots
$$

yield formula (B.9) by choosing $\triangle t \tau\left(T^{3} \delta^{2}\right)^{-1} \leq \kappa$. 
Lemma B.5 (Discrete operations on the weight $\theta$ ). There exists a universal constant $C>0$ uniform with respect to $\triangle t, \delta$ and $T$ such that

$$
\begin{aligned}
& \left|\mathrm{D}_{t}\left(\theta^{\ell}\right)\right| \leq \ell T \mathrm{t}^{-}\left(\theta^{\ell+1}\right)+C \frac{\triangle t}{\delta^{\ell+2} T^{2 \ell+2}}, \quad \ell=1,2, \ldots \\
& \left|\mathrm{t}^{+}\left(\theta^{\prime}\right)\right| \leq T \mathrm{t}^{-}(\theta)^{2}+C \frac{\triangle t}{\delta^{3} T^{4}} \\
& 0 \leq \mathrm{D}_{t}\left(\theta^{\prime}\right) \leq C T^{2} \mathrm{t}^{-}\left(\theta^{3}\right)+C \frac{\triangle t}{\delta^{4} T^{5}}
\end{aligned}
$$

Proof. The proof of (B.12) follows from Taylor formula (B.10) at order $n=2$ and the estimate

$$
\max _{t \in[0, T]} \partial_{t}^{2}\left(\theta^{\ell}\right) \leq \frac{C}{\delta^{\ell+2} T^{2 \ell+2}}, \quad \ell=1,2, \ldots
$$

Inequality (B.13) can be readily deduced from (B.10) at order $n=1$ and using that $\partial_{t} \theta=(2 t-T) \theta$. Finally, estimate (B.14) is consequence of Taylor formula at order $n=2$, estimate (B.11) and the fact $\partial_{t}^{2} \theta \leq C T^{2} \theta^{3}$.

Remark B.6. Some remarks are in order.

- We can directly use Lemmas B.4 and B.5 to obtain estimates for the discrete operators $D_{t}$ and $\mathbf{t}^{ \pm}$ applied to the Carleman weights. Since $r(x, t)$ and $\theta(t)$ are continuously defined for $t \in[-\triangle t, T+\triangle t]$ with $\triangle t$ small enough, formulas (B.9) and (B.12)-(B.14) are equally valid if we sample them at the discrete points $\overline{\mathcal{D}}$, thus providing estimates for operators $\bar{D}_{t}$ and $\overline{\mathrm{t}}^{ \pm}$.

- As it be of interest during the proof of Theorem 1.4, expression (B.9) and the estimates (B.12) and (B.14) are also valid (for a possible different constant $C>0$ but still uniform) if we replace $\mathrm{t}^{-}$by $\mathrm{t}^{+}$everywhere.

\section{References}

[1] O. Bodart, M. González-Burgos, and R. PÉrez-García. Insensitizing controls for a heat equation with a nonlinear term involving the state and the gradient. Nonlinear Anal., 57, 5-6 (2004), $687-711$.

[2] F. Boyer. On the penalised HUM approach and its applications to the numerical approximation of null-controls for parabolic problems. ESAIM Proceedings, 41 (2013), 15-58.

[3] F. Boyer, V. Hernández-Santamaría, And L. De Teresa Insensitizing controls for a semilinear parabolic equation: A numerical approach Math. Control Relat. Fields, 9, 1 (2019), 117-158.

[4] F. Boyer, F. Hubert, and J. Le Rousseau. Discrete Carleman estimates for elliptic operators and uniform controllability of semi-discretized parabolic equations. J. Math. Pures Appl., 93 (2010), no. 3, 240-276.

[5] F. Boyer, F. Hubert, and J. Le Rousseau. Uniform null-controllability for space/time-discretized parabolic equations. Numer. Math., 118 (2011), no. 4, 601-661.

[6] F. Boyer and J. Le Rousseau. Carleman estimates for semi-discrete parabolic operators and application to the controllability of semi-linear semi-discrete parabolic equations. Ann. Inst. H. Poincaré Anal. Non Linéaire, 31 (2014), 1035-1078.

[7] S. ERvedoza And J. Valein. On the observability of abstract time-discrete linear parabolic equations. Rev. Mat. Complut., 23 (2010), no. 1, 163-190.

[8] S. Ervedoza, C. Zheng, and E. Zuazua. On the observability of time-discrete conservative linear systems. J. Funct. Anal., 254 (2008), no. 12, 3037-3078.

[9] C. Fabre, J.P. Puel, And E. Zuazua. Approximate controllability of the semilinear heat equation. Proc. Royal Soc. Edinburgh, 125 A, (1995), 31-61.

[10] E. Fernández-CAra And S. Guerrero. Global Carleman inequalities for parabolic systems and applications to controllability. SIAM J. Control Optim., 45, 4 (2006), 1395-1446. 
[11] E. Fernández-CARA And E. ZuAzuA. Null and approximate controllability for weakly blowing up semilinear heat equations. Ann. I. H. Poincaré-AN, 17, 5 (2000), 583-616.

[12] A. Fursikov and O. Yu. Imanuvilov. Controllability of evolution equations. Lecture Notes, Research Institute of Mathematics, Seoul National University, Korea, (1996).

[13] M. GonzÁlez-Burgos And L. De Teresa. Controllability results for cascade systems of $m$ coupled parabolic PDEs by one control force. Portugal. Math., 67, 1 (2010), 91-113.

[14] M. Gueye. Insensitizing controls for the Navier-Stokes equations. Ann. I. H. Poincaré-AN, 30, 5 (2013), 825-844.

[15] G. Lebeau and L. Robbiano. Contrôle exact de l'équation de la chaleur. Comm. Partial Differential Equations, 20 (1995), no. 1-2, 335-356.

[16] G. Lebeau AND E. ZuazuA. Null-controllability of a system of linear thermoelasticity. Arch. Rational Mech. Anal., 141 (1998), no. 4, 297-329.

[17] J.-L. Lions. Quelques notions dans l'analyse et le contrôle de systèmes à données incomplètes. Proceedings of the XIth Congress on Differential Equations and Applications/First Congress on Applied Mathematics, University of Málaga, 1990, 43-54.

[18] P. Lissy, Y. Privat, And Y. Simpore. Insensitizing control for linear and semi-linear heat equations with partially unknown domain. ESAIM Control Optim. Calc. Var., (2018). https: //doi.org/10.1051/cocv/2018035.

[19] L. DE TERESA. Insensitizing controls for a semilinear heat equation. Comm. Partial Differential Equations, 25, 1-2 (2000) 39-72.

[20] L. De Teresa And E. Zuazua. Identification of the class of initial data for the insensitizing control of the heat equation. Commun. Pure. Appl. Anal., 8, 1 (2009) 457-471.

[21] D. Xu. On the Observability of Time Discrete Integro-differential Systems. Appl. Math. Optim., (2019). https://doi.org/10.1007/s00245-019-09556-3.

[22] C. Zheng. Controllability of the time discrete heat equation. Asymptot. Anal., 59 (2008), no. 3-4, 139-177.

[23] X. Zhang C. Zheng, and E. Zuazua. Time discrete wave equations: boundary observability and control. Discrete Contin. Dyn. Syst., 23 (2009), no. 1-2, 571-604. 